\title{
Dynamics and fitness benefits of male- male sociality in wild Guinea baboons (Papio papio)
}

\author{
Dissertation \\ for the award of the degree \\ "Doctor of Philosophy (Ph.D.)" \\ Division of Mathematics and Natural Sciences \\ of Georg-August-Universität Göttingen
}

within the doctoral program

Behavior and Cognition (BeCog)

at the Georg-August University School of Science (GAUSS)

Submitted by

Federica Dal Pesco

from Bologna, Italia

Göttingen - 2019 



\title{
Dynamics and fitness benefits of male- male sociality in wild Guinea baboons (Papio papio)
}

\author{
Dissertation \\ for the award of the degree \\ "Doctor of Philosophy (Ph.D.)" \\ Division of Mathematics and Natural Sciences \\ of Georg-August-Universität Göttingen
}

within the doctoral program

Behavior and Cognition (BeCog)

W WW at the Georg-August University School of Science (GAUSS) 

Doctoral Thesis Committee:

Prof. Dr. Julia Fischer (Advisor)

Cognitive Ethology Laboratory

German Primate Center, GmbH

Kellnerweg 4, 37077 Göttingen, Germany

Dr. Oliver Schülke

Behavioral Ecology

Johann-Friederich-Blumenbach-Institute for Zoology \& Anthropology

Kellnerweg 6, 37077 Göttingen, Germany

Dr. Tanya Behne

Developmental Psychology

Georg-Elias-Institute of Psychology, Göttingen University

Waldweg 6, 37073 Göttingen, Germany

Defense Board Members:

Prof. Dr. Julia Fischer (First Referee)

Cognitive Ethology Laboratory

German Primate Center, GmbH

Kellnerweg 4, 37077 Göttingen, Germany

Dr. Oliver Schülke (Second Referee)

Behavioral Ecology

Johann-Friederich-Blumenbach-Institute for Zoology \& Anthropology

Kellnerweg 6, 37077 Göttingen, Germany

Dr. Tanya Behne

Developmental Psychology

Georg-Elias-Institute of Psychology, Göttingen University

Waldweg 6, 37073 Göttingen, Germany

Prof. Dr. Julia Ostner

Behavioral Ecology

Johann-Friederich-Blumenbach-Institute for Zoology \& Anthropology

Kellnerweg 6, 37077 Göttingen, Germany

PD Dr. Christian Roos

Primate Genetics Laboratory

German Primate Center, GmbH

Kellnerweg 4, 37077 Göttingen, Germany

Prof. Dr. Annekathrin Schacht

Affective Neuroscience and Psychophysiology

Georg-Elias-Müller-Institute of Psychology

Goßlerstraße 14, 37073 Göttingen, Germany

Date of the oral examination: 25th of April 2019 

Herewith I declare that I have written this thesis independently and with no other aids or sources than those quoted.

Göttingen, 29th of March 2019

Federica Dal Pesco 

To my parents, Laura \& Walther,

who always encouraged me to challenge myself and live life to the fullest!

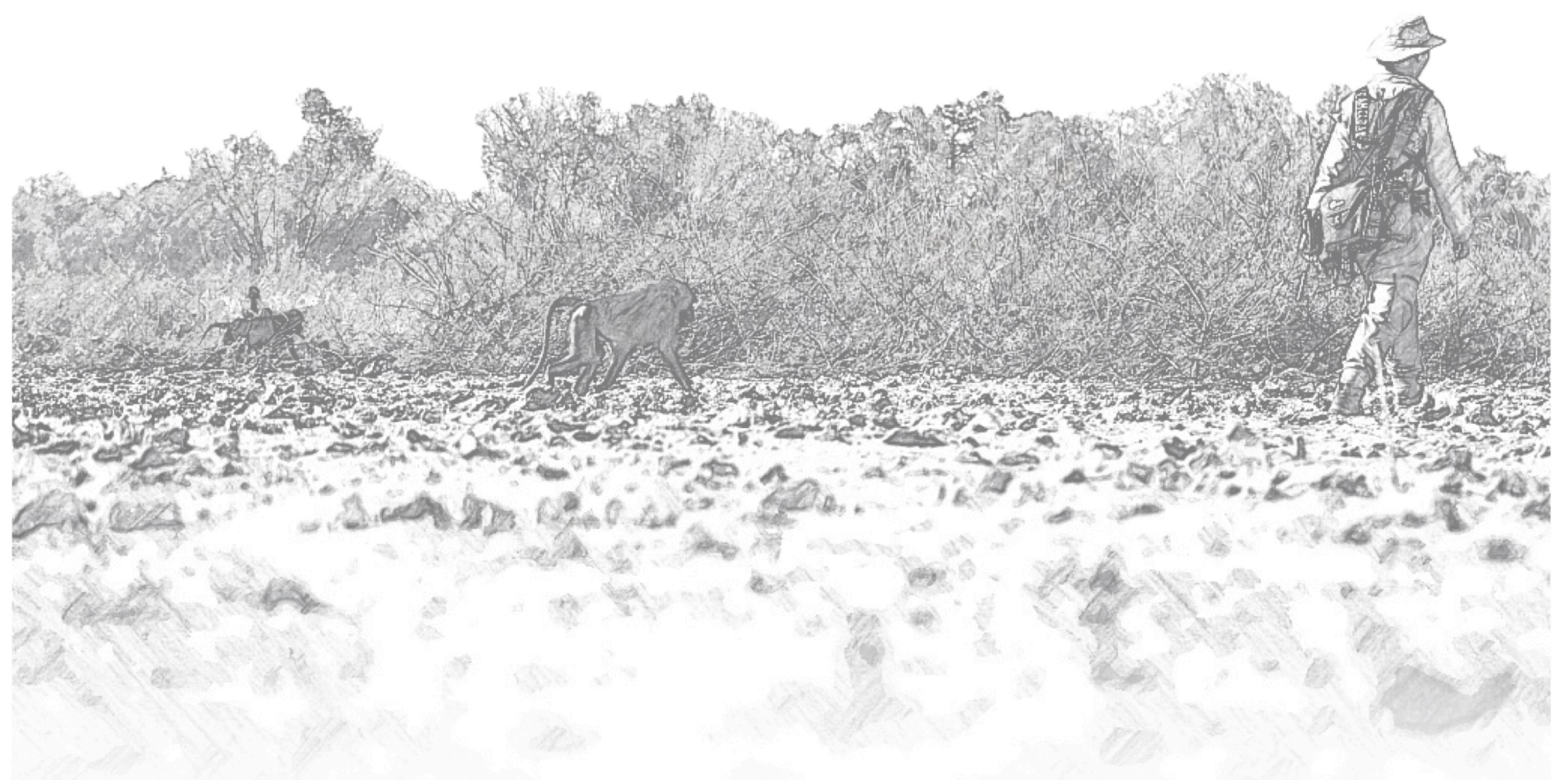





\section{Table of Contents}

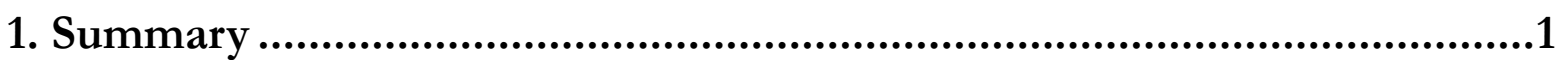

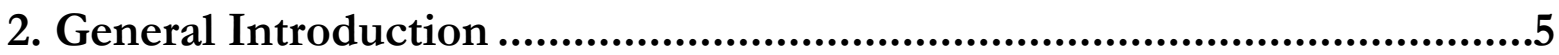

2.1 The evolution of group-living ................................................................................................6

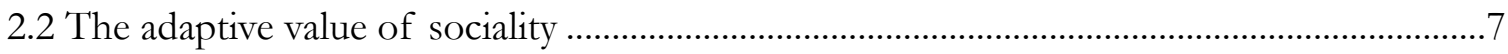

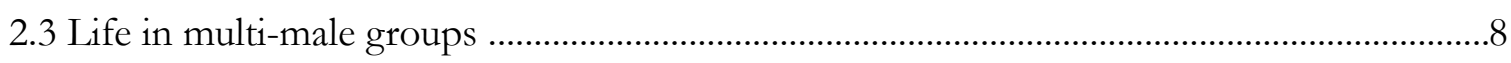

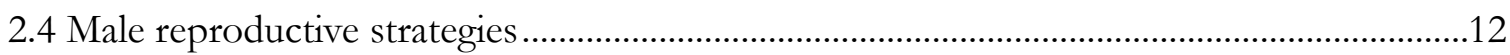

2.5 Male bonding and male-male ritualized greeting behavior...................................................15

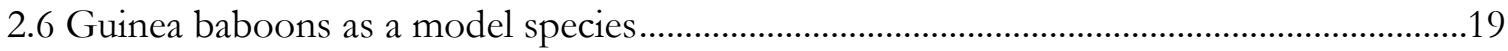

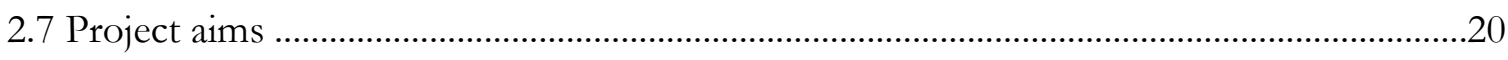

3. Study I ................................................................................23

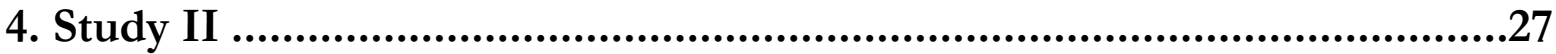

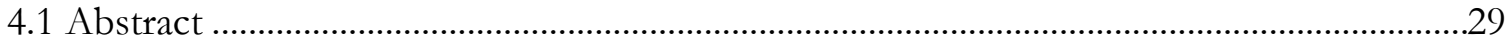

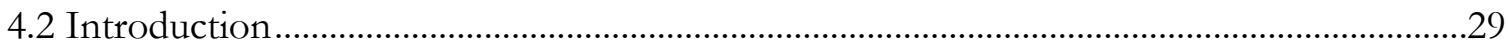

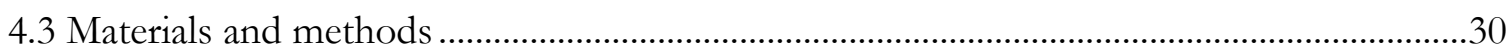

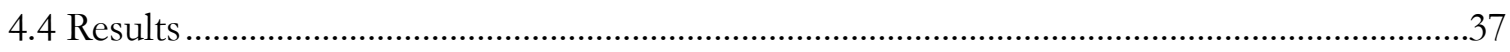

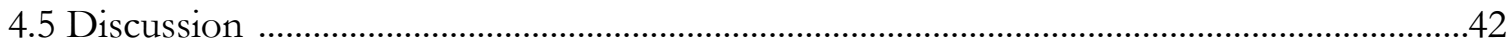

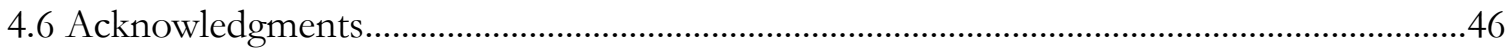

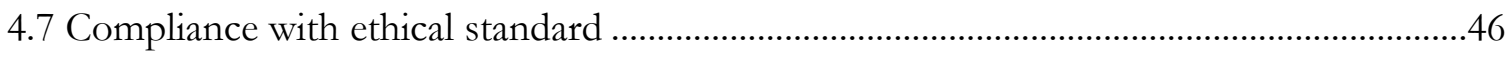

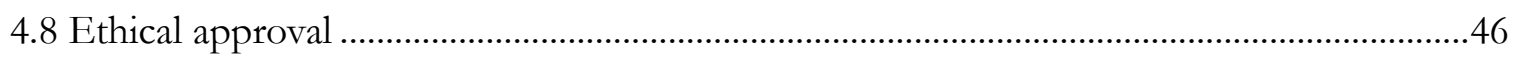

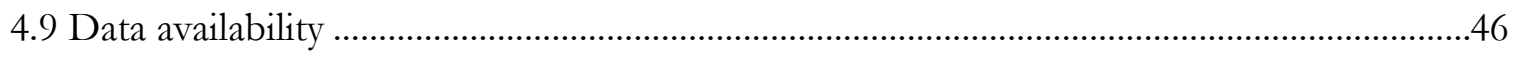

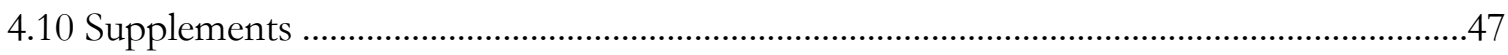

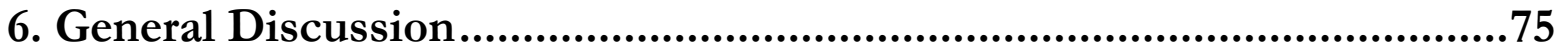

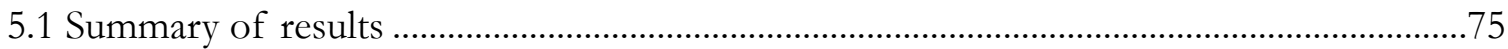

5.2 Ritualized greeting behavior in a tolerant multilevel society ................................................76

5.3 The tolerant baboon: male-male sociality, kinship, and competition ..................................77 
5.4 The adaptive benefits of male-male sociality

5.5 The female perspective and the influential role of pair bonds .83

5.6 Male-male relationship dynamics and potential benefits. .85

5.7 An evolutionary perspective... . .87

7. Conclusions and Outlook .93

8. References............................................................................97

9. Table of Figures ...................................................................121

10. Table of Tables ............................................................................123

11. Acknowledgments.................................................................127

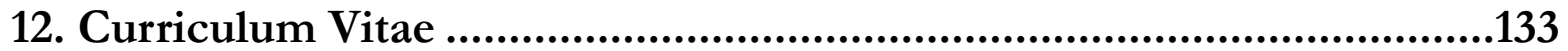





\section{Summary}

Males living in multi-male groups display a wide range of relationships with co-resident males, varying from high levels of competition, intolerance and avoidance to cooperation, affiliation and social bonds. Despite the wide diversity of male-male relationship dynamics, the existence of multi-male associations and male-male bonds is puzzling, as males compete over un-sharable fertilization. Multimale associations can emerge as a result of increased between-group competition, which would necessitate communal defense of females. In groups characterized by stable male co-residence, social bonds are thought to develop in scenarios of medium to low within-group contest potential, which would make it feasible for males to invest in coalitionary support aimed at rank ascension. Accordingly, multi-male alliances and coalitions between strongly bonded males are linked to lower takeover likelihoods and/or enhanced male reproductive success in several mammalian species. Female choice can also play a role, with increased reproductive success for males that invest in heterosexual bonds and provide services like protection from harassment and infanticide, assistance in rearing young, and support in conflicts. In this context, male-male ritualized behaviors are crucial behavioral mechanisms that balance the trade-offs imposed by male co-residence and help regulate male-male relationships. These ritualized exchanges are hypothesized to function as a tension buffering mechanism during competitive contexts and/or as honest signals used to test relationship quality, maintain social bonds, and promote cooperation. Several findings suggest that greeting function varies from species to species and that, in some cases, this can be influenced by the level of male tolerance and cooperation.

In this thesis I investigate the dynamics and fitness benefits of male-male sociality in wild Guinea baboons by exploring the presence of strong male-male bonds along with their effect on male reproductive success and the function of ritualized greeting behavior between males. Guinea baboons live in nested multilevel societies, with several "units" forming a "party", which regularly aggregate into "gangs". Males engage in highly ritualized greetings with an intense repertoire involving potentially harmful behaviors like genital fondling, which may be crucial to regulating social dynamics in such large and fluid communities. The presence of male-male affiliation and cooperation, combined with the relatively high level of female leverage in intersexual relationships, make this species an intriguing model to assess how the advantages conferred by male-male sociality play out in a highly tolerant multilevel system. I examine whether number and strength of social bonds and number of associated secondary males are associated with higher average relatedness or enhanced reproductive success, using social behavior, genetic relatedness, and paternity of 24 adolescent and adult males. I 
also investigated the function of ritualized greetings and whether they are used to buffer tension and/ or assess relationship quality.

In chapter 3, I show Guinea baboon greetings are crucial in the regulation of male social dynamics by signaling social levels and party membership. I present evidence that within-party greetings are honest affiliative signals that test relationship quality with spatially tolerant partners, while accentuating relationship strength with strongly bonded partners. All my analyses show greetings are not used to buffer tension in this tolerant social system, and describe how they are characterized by high levels of reciprocity, physical contact, and intensity. Lastly, I discuss how these findings are in sharp contrast to reports from other members of the genus Papio, which present lower levels of male tolerance and cooperation, while parallel findings are reported for other more tolerant and cooperative species.

In chapter 4, I show that within-party male-male affiliative relationships are differentiated and stable over time, indicating male Guinea baboons form strong bonds. I demonstrate that strongly bonded males are more likely to support each other in coalitions and have higher average relatedness. This suggests kinship may have facilitated the development of bonds and cooperation. Interestingly though, bonds are neither restricted to kin nor does kinship guarantee stronger bonds, indicating that other mechanisms like familiarity may play a more central role in male-male sociality. I show that the distribution of reproduction at the party level is not different from chance, while primary males sire $89.5 \%$ of offspring at the unit level. Contrary to other species, all three measures of sociality are not positively linked with short-term reproductive success either through number of associated females or sired offspring. Instead, males with stronger bonds actually have significantly fewer associated females. A post-hoc analysis shows that preferred partners are stable over time, but that the proportion of time males invest in socializing with other males is negatively affected by the number of associated females. Thus, male Guinea baboons appear to face a trade-off between investing in same-sex bonds and heterosexual ones.

My thesis is the first systematic analysis to demonstrate how co-resident males regulate relationships through ritualized greetings and contribute to our understanding of the dynamics and fitness benefits of male-male sociality in this multilevel and tolerant system. I contribute to the theory that ritualized behaviors and rituals developed in parallel with a transition from more aggressive social styles to more tolerant and cooperative ones. Future systematic comparative studies will be key to understanding if these patterns hold across the order Primates. While strong bonds between male Guinea baboons share characteristics with those of other primates, contrary to evidence from several species my findings show sociality need not be directly linked to increased short-term reproductive success. Investigating if and how male-male sociality confers benefits to reproduction over the long-term and advantages beyond reproductive success represent crucial steps for future research. 


\section{General Introduction}

Long-term investments in affiliative and cooperative social relationships represent crucial strategies towards mitigating the costs of group-living (reviewed in Ostner and Schülke, 2018; Thompson, 2019). Such social relationships and bonds, as well as their adaptive benefits, have been broadly investigated in females (Silk, 2007a). Notwithstanding recent research on male-male affiliation and cooperation, however, male social relationships remain less clear (Hill and van Hooff, 1994). As inclusive fitness is assumed to have directed the evolution of female cooperation and bonds (reviewed in Silk, 2007b), this research bias is often attributed to the predominance of female philopatry in many social mammals, including numerous primates, which sets the scene for male dispersal (reviewed in CluttonBrock, 2016). Studies also argue that affiliation and cooperation are more likely to occur among females, who compete for shareable resources such as food, whereas males compete for un-shareable resources such as fertilization (Hill and van Hooff, 1994). Arguing that male cooperation and bonds are rare and tend to occur exclusively between kin (van Hooff and van Schaik, 1994), early studies focused on competition, dominance, and aggression (Hill and van Hooff, 1994). However, males can display a broad variety of relationship styles when interacting with other males.

Investigations of male-male interactions have moved beyond competition in recent decades. Tolerance, cooperation, affiliative interactions and social bonds between males have been reported in several species both between kin and non-kin (e.g. African lions, Panthera leo: Grinnell et al., 1995; bottlenose dolphins, Tursiops sp.: Krutzen et al., 2003; chimpanzees, Pan troglodytes: Mitani, 2009; Guinea baboons, Papio papio: Patzelt et al., 2014; Barbary macaques, Macaca sylvanus: Young et al., 2014a; Assamese macaques, Macaca assamensis: Kalbitz et al., 2016). Males have also evolved ritualized affiliative behaviors to balance the trade-offs imposed by co-residence. These interactions can vary greatly in their features and function and are crucial to help regulate male-male relationships in multi-male groups (Whitham and Maestripieri, 2003). Although these recent developments improve our understanding of male-male sociality, our knowledge remains limited compared to females and new findings would advance our understanding of the proximate and ultimate mechanisms of such relationships.

In the following sections, I summarize the evolution of group-living (2.1) and the adaptive benefits of sociality (2.2). The next section describes the development of multi-male groups considering both male and female perspectives (2.3), followed by male reproductive competition over female monopolization as well as alternative mating strategies (2.4). This is followed by the features and adaptive benefits of male-male social bonds, their evolution, and underlying mechanisms (2.5) as well as male- 
male ritualized behaviors and their function in regulating relationships (2.5). I finally introduce and highlight why Guinea baboons are an ideal and intriguing model for studying the dynamics and fitness benefits of male sociality (2.6), and conclude with the aims, hypotheses, and predictions addressed in this thesis (2.7).

\subsection{The evolution of group-living}

Living in groups can entail significant individual costs, including increased risk of disease transmission (Freeland, 1976; Altizer et al., 2003; Kappeler et al., 2015) and competition over food, territories, sleeping sites, or mates (Alexander, 1974; Emlen and Oring, 1977; Krause and Ruxton, 2002). In spite of these costs, group-living or gregariousness has evolved in insects (Queller et al., 2000; Hughes et al., 2002; Leadbeater and Chittka, 2009), fishes (Wong et al., 2004; Jordan et al., 2010; Heathcote et al., 2017), birds (Radford, 2008; Braun and Bugnyar, 2012; Aplin et al., 2014) and various mammalian species (Rubenstein and Hack, 2004; Boyd, 2006; Mosser and Packer, 2009; Mitani et al., 2012; Carter and Wilkinson, 2015; Connor and Krützen, 2015). While almost a quarter of mammals are groupliving (Lukas and Clutton-Brock, 2013), it is in the order Primates that an exceptionally high proportion of species live in groups (van Schaik and Kappeler, 1997; Kappeler and van Schaik, 2002). Thus, investigating the evolution of group-living has been a major focus for both evolutionary ecology and primatology.

The costs imposed by gregariousness can be mitigated by its advantages. Individuals can greatly benefit from stable associations with conspecifics in the form of lower predation risk through dilution effects and communal defense (Williams, 1966; Hamilton, 1971; Alexander, 1974; Rubenstein, 1978; van Schaik, 1983), increased access to resources via cooperative hunting (Packer et al., 1990; Boesch, 1994; Creel and Creel, 1995; Packer and Ruttan, 1998), and the potential for joint territory and resource defense (Emlen and Oring, 1977; Wrangham, 1980; Feh, 1999; Williams et al., 2004; Mosser and Packer, 2009; Scarry, 2013). Gregariousness can also benefit individuals via increased opportunities for social thermoregulation via huddling and grooming to help mitigate heat loss in cold environments (Andrews and Belknap, 1986; Satinoff, 2011; Terrien et al., 2011; McFarland et al., 2015, 2016; Henzi et al., 2017). Ultimately, stable group associations evolve when the benefits of living in groups outweigh the costs of sharing space and resources (Krebs and Davies, 1993; Krause and Ruxton, 2002).

A group or social unit is usually defined as a set of conspecific individuals who associate and interact regularly and do so more frequently than they do with conspecifics of other groups (Struhsaker, 1969; Krause and Ruxton, 2002). In many species this results in stable social relationships and cooperation (Silk et al., 2010a) that can develop into social bonds over time (Silk, 2002). In order to cope with the trade-offs imposed by gregariousness, individuals adopt behavioral strategies to maximize benefits and fitness. These strategies result in a broad diversity of social systems where differences in 
group size, composition and spatiotemporal cohesiveness (i.e. social organization) are interrelated with variations in social structure (i.e. who interacts and has relationships with whom?) and mating system (i.e. who mates with whom?) (Kappeler and van Schaik, 2002). Males and females face different socioecological pressures and are therefore confronted with different sets of trade-offs. While female reproductive success is largely affected by feeding competition (Wrangham, 1980; Sterck et al., 1997; Koenig, 2002) and infanticide risk (Sterck et al., 1997; Palombit, 1999), male fitness is shaped by access to females and fertilization (Emlen and Oring, 1977; van Hooff, 2000). Due to these different selective pressures, social systems result in complex interactions between the distinct behavioral strategies of males and females.

\subsection{The adaptive value of sociality}

Group-living individuals repeatedly interact with other group members via affiliative/agonistic interactions that, over time, lead to the development of dyadic social relationships with different tenor (Hinde, 1976; Silk et al., 2013). Affiliative relationships are considered social bonds when they are stronger than others within the same group, stable over time, and equitable between partners (Silk 2002). Social bonds were first characterized in studies on philopatric female baboons (Silk et al., 2006a, 2006b) that determined correlations with short-term variations in well-being (measured as glucocorticoid elevations in response to stressors: Engh et al., 2006a; Crockford et al., 2008; Wittig et al., 2008) and long-term variations in fitness outcomes (Silk, 2007a). Similar differentiated relationships are reported in several other mammals (e.g. feral horses, Equus caballus: Cameron et al., 2009; bottlenose dolphins: Frère et al., 2010; yellow-bellied marmots, Marmota flaviventris: Wey and Blumstein, 2012) as well as in birds (e.g. common ravens, Corvus corax. Fraser and Bugnyar, 2010; Braun and Bugnyar, 2012; greater ani, Crotophaga major: Riehl and Strong, 2018) and fishes (e.g. guppies, Poecilia reticulata: Heathcote et al., 2017).

By now the adaptive value of differentiated social ties is apparent throughout mammalian species (reviewed in Ostner and Schülke, 2018; Thompson, 2019) including humans (Homo sapiens: Holt-Lunstad et al., 2010, 2015). Nevertheless, the diversity of methodologies used to measure sociality makes it difficult to draw definitive conclusions about the relative importance of these various measures of sociality (Ostner and Schülke, 2018; Silk et al., 2018). Affiliation strength or bonds strength between females is associated with enhanced reproductive success (e.g. house mice, Mus musculus: Weidt et al., 2008; feral horses: Cameron et al., 2009; bottlenose dolphins: Frère et al., 2010), higher offspring survival (e.g. yellow baboons, Papio cynocephalus: Silk et al., 2003, chacma baboons, Papio ursinus: Silk et al., 2009; bottlenose dolphins: Frère et al., 2010) and longer life spans (e.g. chacma baboons: Silk et al., 2010b; yellow baboons: Archie et al., 2014). In Assamese macaques, the strength of male-male social bonds is associated with enhanced male reproductive success (Schülke et al., 2010). Measures of social integration based on the quantity of social partners are also linked to individual fitness. The 
number of strong social bonds is associated with higher birth rates in female chacma baboons (McFarland et al., 2017), increased survival during harsh winters in Barbary macaques (McFarland and Majolo, 2013; see also Lehmann et al., 2016), and better thermoregulation in female vervet monkeys (Chlorocebus pygerythrus: McFarland et al., 2015). A single study on chacma baboons identified a link between number of weak social bonds and higher offspring survival (McFarland et al., 2017). The relative importance of quantity of partners and weak ties, however, has been debated as both measures are heavily affected by sampling effort and group size (Silk et al., 2018). Measures of indirect connectedness have also been used successfully to link sociality with various fitness correlates (e.g. Stanton and Mann, 2012; Brent et al., 2013; Gilby et al., 2013; Cheney et al., 2016; Ellis et al., 2017). Altogether this evidence emphasizes the need for comprehensive analyses targeted at understanding which features are linked to which fitness benefits and if, when, and why different aspects of sociality play a role in different animal societies (Ostner and Schülke, 2018; Silk et al., 2018).

In spite of the potential benefits of sociality, these can be conditional on specific circumstances and social ties can even have detrimental effects in some species. Offspring of strongly bonded females in white-faced capuchin monkeys have increased survivorship during demographically stable periods, but they are less likely to survive during alpha-male replacement events (Cebus capucinus imitator: Kalbitzer et al., 2017). In blue monkeys, the risk of mortality is increased for females that invest in strong bonds with inconsistent partners, indicating that social relationships can be costly when unstable over time. Over multiple years this strategy can result in higher mortality than for females that invest in either consistent or inconsistent but weaker bonds (Cercopithecus mitis stublmanni: Thompson and Cords, 2018). In yellow-bellied marmots, a facultatively social mammal, affiliation strength between females is negatively associated with reproductive success and individuals with stronger ties have reduced longevity and are more likely to die during hibernation (Wey and Blumstein, 2012; Yang et al., 2016; Blumstein et al., 2018). These results suggest that sociality is not universally beneficial and individuals likely need to balance the benefits and costs of these relationship investments.

\subsection{Life in multi-male groups}

As males compete over exclusive access to breeding females, multi-male groups are puzzling considering the costs of mating competition and potential repercussions on individual reproductive success (Kappeler, 1999; Clutton-brock and Isvaran, 2006). The costs of these associations can be small for males living in all-male or bachelor groups, or in groups where other males are not sexually mature or are related to females (reviewed in Clutton-Brock, 2016). Nevertheless, dominant males can pay a high price in the form of loss of reproduction when living in bisexual groups with sexually mature subordinates (reviewed in Clutton-Brock, 2016). Understanding variations in group composition and how multi-male associations have evolved is one of the crucial questions of socioecological studies (Kappeler, 1999). Are dominant males simply unable to exclude others from accessing females, or are 
these associations a product of "reproductive transactions" between males? To what extent are these associations a product of specific individual socio-sexual behavioral strategies? The next sub-sections address these questions and the benefits males and females derive from multi-male associations.

\subsubsection{Benefits of "supernumerary males": male and female perspectives}

Males should generally prefer to live in single-male groups (Clutton-brock and Isvaran, 2006). Multimale groups should be favored only when associations between males result in their increased fitness (Kappeler, 1999; but see evolutionary demographic models e.g. Port and Johnstone, 2013). Such fitness benefits can include access to more females per male, higher female reproductive success, and increased survival for males and their sired offspring. From a female perspective, multi-male associations can be beneficial, with males providing "social services" that help mitigate socioecological pressures such as predation, inter-group feeding competition, harassment from other males, and infanticide (Kappeler, 1999).

The trade-offs faced by males with regard to group composition (i.e. single- vs. multi-male groups) can be affected by socioecological factors, including predation pressure and inter-group competition. Groups with several adult males are common when male population density is high and competition is intense (Pope, 1990), or when predation pressure is increased (van Schaik and Hörstermann, 1994; Stanford, 1998). Males are crucial to group protection from predators through higher levels of vigilance, better predator detection, and more engagement in active defense than females (van Schaik and van Noordwijk, 1989; Rose, 1994; Stanford, 1998). Thus, higher numbers of males can directly benefit themselves, their offspring and females. Male vigilance can also be directed at male activities in neighboring groups (Baldellou and Henzi, 1992; Steenbeek et al., 1999) and thereby be increased when home-ranges overlap (Steenbeek et al., 1999). Males jointly defend group territory/home range from conspecifics and play a key role in inter-group encounters (Perry, 1996; Kitchen, 2004; Zhang et al., 2006; Talebi et al., 2009; Garber and Kowalewski, 2011; Markham et al., 2012; Wilson et al., 2012). As the number of adult males in the group can affect winning probabilities during intergroup conflicts (Kitchen, 2004; Kitchen et al., 2004; Markham et al., 2012; Wilson et al., 2012; Scarry, 2013), multi-male associations can greatly benefit males as well as all other group members.

Males mainly participate in inter-group aggression for mate defense (Trivers, 1972; Emlen and Oring, 1977). They defend their mates directly by fighting and chasing away male neighbors and by herding females to prevent interactions with non-resident males or transfers to other groups (Sicotte, 1993; Swedell, 2000; Fashing, 2001; Kitchen et al., 2004). Encounter intensity and number of participating males are associated with the presence of estrous females (Kitchen et al., 2004), which are most often the receivers of herding behaviors (Sicotte, 1993). By directly defending mates from non-resident harassing males, resident males also chase away neighboring groups and defend food resources, which benefits females and other group members indirectly ("hired-guns"-hypothesis: Wrangham and 
Rubenstein, 1986; also reviewed in Fashing, 2001). Males can also directly defend food resources to attract mates and enhance female reproductive success and offspring survival (reviewed in Fashing, 2001). Although this dichotomy is often difficult to disentangle (Richter et al., 2016), male communal defense is nevertheless associated with both territory expansion and increased access to food resources (Fashing, 2001; Williams et al., 2004; Crofoot, 2007; Harris, 2010; Mitani et al., 2010; Scarry, 2013, 2017; Richter et al., 2016), which results in higher female reproductive rates/fecundity (Williams et al., 2004; Richter et al., 2016; see also Langergraber et al., 2017). Recent studies show that male resource defense can also emerge as a by-product of intersexual cooperation in species where females play an active role in intergroup conflicts. In vervet monkeys, males support female instigators against simulated intruders, which can increase their social status as cooperative partners and enhance their mating success (Arseneau et al., 2015; Arseneau-Robar et al., 2016).

Multi-male groups are also crucial to offspring survival through male vigilance and communal defense by increasing protection from harassment and infanticide by neighboring males (Sicotte, 1993; Robbins, 1995; Steenbeek et al., 1999) and females (Mosser and Packer, 2009). Accordingly, males respond more strongly to intruders when younger offspring were present and when calls simulated males likely to be infanticidal (Kitchen, 2004; Wich et al., 2004; Arseneau et al., 2015). Male care for infants can result in enhanced male reproductive success with males affiliating at higher rates with all infants (regardless of paternity) siring more offspring (Rosenbaum et al., 2018) and can even influence female fitness through male assistance in multi-male groups (Wright, 1990).

\subsubsection{Male reproductive transactions and monopolization potential}

Although the presence of supernumerary males can benefit both males and females, it is a matter of debate to what extent male co-residence is due to limitations on male monopolization potential or a product of male reproductive transactions. Transactional models presume that male composition is the direct outcome of negotiations between resident males to maximize group stability and related benefits (reviewed in Port and Kappeler, 2010; see also Port et al., 2018). Limited control models (also "tug-of-war models") instead argue that dominant males have limited control over monopolization and that co-residence results from dominant individuals' inability to prevent subordinates from mating (Reeve et al., 1998). Both models predict similar outcomes in the division of reproduction within the group, making a clear distinction very difficult (Clutton-Brock, 1998; also see Reeve and Keller, 1998). The following two paragraphs introduce these two models, considering their theoretical and empirical basis as well as their limitations.

Transactional models argue that reproduction can be controlled and co-resident males share reproduction (via concession or restraint) in exchange for mutual benefits (Reeve et al., 1998). According to the concession model, dominant males actively share just enough reproduction to keep subordinates from leaving the group while benefitting from their support (Reeve and Ratnieks, 1993; Clut- 
ton-Brock, 1998). In the restraint model, subordinates are able to claim unsanctioned portions of reproduction while exhibiting reproductive restraint to avoid eviction from the group (Johnstone and Cant, 1999). The magnitude of concession/restraint is determined by kinship and other social and ecological factors (Reeve and Keller, 1998). Several studies report evidence for reproductive transactions in primates (e.g. mountain gorillas, Gorilla beringei: Stoinski et al., 2009; geladas, Theropithecus gelada: Snyder-Mackler et al., 2012; chimpanzees: Bray et al., 2016) and other mammals (e.g. dwarf mongooses, Helogale parvula: Creel and Waser, 1991; Keane et al., 1994; African lions: Packer et al., 1991). However, these studies provide no conclusive support as these findings do not preclude alternative interpretations (Clutton-Brock, 1998) and most studies fail to fully test predictions (Port et al., 2018). Concession models, additionally, are of limited value in the primate order as their assumptions are not likely to apply to male primates, where reproduction is unlikely to be controlled by the dominant male alone, sperm competition decreases the ability to assess the value of reproductive exchanges, and low reproductive rates make fine-tuned adjustments of skew more difficult (Port and Kappeler, 2010).

Multi-male associations can alternatively be explained by dominant males' limited control over monopolization (Reeve et al., 1998). Indeed, competing for exclusive access to fertile females can be too costly for males or otherwise limited by other factors (Mitani et al., 1996). Female number and distribution significantly impact monopolization success (Mitani et al., 1996) and set the stage for male mate competition type (i.e. contest/scramble competition: van Schaik, 1989; reviewed in Alberts, 2012). The number of resident females is positively associated with the number of co-resident males (Mitani et al., 1996; Nunn, 1999a; Carnes et al., 2011) and a decrease in monopolization success (Kutsukake and Nunn, 2006). Although absolute size of female groups seems to set the limit for male monopolization, other factors such as group cohesiveness (van Schaik and van Hooff, 1983), visibility (Rowell, 1988), breeding seasonality (Carnes et al., 2011; also see Dunbar and Srivastava, 1996), and estrous synchrony (Nunn, 1999a; Ostner et al., 2008b; Carnes et al., 2011) also play a key role. The success of male monopolization strategies, however, also directly depends on the ability to correctly detect ovulation. This is contingent on the presence of signals that indicate female receptivity (e.g. perineal swellings: Nunn, 1999b) and, most importantly, the accuracy with which these signals indicate the exact timing of ovulation (Nunn, 1999b; Higham et al., 2008; Douglas et al., 2016; Street et al., 2016).

In sum, while both models can theoretically play a role in the emergence of multi-male groups, in the primate order more evidence has accumulated in support of males' limited monopolization potential. Thus, as introduced in the following section, limited male monopolization results in different male reproductive strategies, comprising both male direct competition and alternative reproductive strategies (Alberts, 2012). 


\subsection{Male reproductive strategies}

Males and females compete over different fitness-limiting resources and, in most species, this leads to distinct reproductive strategies (Trivers, 1972). Females can reproduce with only one male at a time and provide most of the parental care through long gestation and lactation periods. Their reproductive success relies on choosing a high quality mate that provides either direct benefits through food, protection, or parental care or indirect benefits through high quality and compatible genes (reviewed in Kappeler, 2012). Male reproductive success theoretically depends on the successful fertilization of the highest possible number of females for the longest possible time (Trivers, 1972). Thus, male reproductive success is associated with time of male sexual maturation and longevity or, more specifically, the tenure or breeding lifespan (reviewed in Alberts, 2012). Primate males have evolved various strategies to balance the maximization of their reproductive output and the potential reproductive loss imposed by other males. These strategies can lead to skewed breeding distributions, with high reproductive skew corresponding to high monopolization by a single male (Keller and Reeve, 1994). Ultimately, male reproductive success depends on the interplay between female mate choice, malemale direct competition, and alternative male reproductive strategies (Alberts, 2012).

\subsubsection{Competition, dominance and priority of access}

Within-group male-male competition leads to the establishment of dominance hierarchies, within which individuals are ranked based on fighting ability and outcomes of aggressive interactions (Drews, 1993). While there is overwhelming evidence that dominance rank is linked to monopolization and reproductive success (Dewsbury, 1982; Cowlishaw and Dunbar, 1991; Ellis, 1995; Kutsukake and Nunn, 2006; Majolo et al., 2012), male reproductive skew can vary considerably (Cowlishaw and Dunbar, 1991; Bulger, 1993; Alberts et al., 2003, 2006). The priority of access model attempts to explain how reproduction is allocated between co-resident males (Altmann, 1962), with higher ranking males (i.e. alpha-males) assumed to have priority over mating. As only one female can be monopolized at a time, the proportion of mating lost to subordinates should depend on the degree of female reproductive synchrony (Altmann, 1962). Several studies support these predictions (Pope, 1990; Cowlishaw and Dunbar, 1991; Bulger, 1993; Alberts et al., 2006; Boesch et al., 2006b; Ostner et al., 2008a; Bissonnette et al., 2011; Young et al., 2013; Bray et al., 2016; but see Newton-Fisher et al., 2010; Dubuc and Muniz, 2011) and indicate that the number of co-resident males has a negative effect on the alpha-male's mating or reproductive success (Cowlishaw and Dunbar, 1991; Alberts et al., 2003, 2006; Boesch et al., 2006b; Kutsukake and Nunn, 2006; Ostner et al., 2008b; Bray et al., 2016), either due to reduced power differentials or increased frequency of challenges (Cowlishaw and Dunbar, 1991).

Male dominance rank and related fitness benefits are generally correlated with physical attributes such as body size, weight, canine size, and health (Plavcan, 1993; Plavcan et al., 1995; Leigh et al., 2008; 
Galbany et al., 2015; Georgiev et al., 2015; also reviewed in Clutton-Brock, 2016). Likely due to such physical attributes, age-related physical abilities also influence male rank. Subadult males generally rank lower than adults, adult males in prime physical condition occupy the highest positions, and rank declines consistently with age thereafter (Bercovitch et al., 2003; Widdig et al., 2004; Setchell et al., 2005; Alberts et al., 2006). Rank does not, however, exclusively depend on physical strength and fighting abilities (de Waal, 1985). Social support by group members, and female behavior can impact rank and reproductive success (Furuichi, 1989; Bulger, 1993; Alberts et al., 2003, 2006; Dubuc and Muniz, 2011; Young et al., 2013; Markham et al., 2015). Coalition formation between males is reported in most genera that form multi-male groups (Bissonnette et al., 2014). However, coalition frequency varies greatly across species and coalitionary support is most prevalent in groups with larger numbers of co-resident males and a smaller than expected share of mating for the alpha male(Bissonnette et al., 2014). Male coalitionary support has been associated with rank ascension and enhanced reproductive success in several species (e.g. Schülke et al., 2010; Gilby et al., 2013). Coalitionary strategies may also be age-dependent in some species (Noë, 1992; Bissonnette et al., 2009; Rathke et al., 2017) and aid post-prime males compensate for the decline in fighting abilities and mating success (Bercovitch, 1988; Noë, 1992; Kuester et al., 1995; Bissonnette et al., 2009, 2011). While physical attributes and direct competition are factors relevant to male reproductive success, coalitionary support and other reproductive strategies can greatly influence rank and reproductive success, leading to the adoption of a variety of such alternative strategies.

\subsubsection{Alternative reproductive strategies}

In addition to direct mating competition, males can adopt a series of alternative reproductive strategies that can impact reproductive skew. Subordinate males can engage in opportunistic "sneaky" copulations that can result in paternities (Berard et al., 1994; Launhardt et al., 2001; Soltis et al., 2001; Alberts et al., 2006; Modolo and Martin, 2008). Alternatively, male investment in heterosexual friendships can result in mutual benefits in the form of increased male mating success (Kulik et al., 2012; Massen and Vries, 2012; Ostner et al., 2013; Städele et al., 2019), enhanced infant protection and parental care (Palombit et al., 1997; Buchan et al., 2003; Moscovice et al., 2009, 2010; Nguyen et al., 2009; Huchard et al., 2010; Ostner et al., 2013; Baniel et al., 2016; Städele et al., 2019), and male services toward females such as reduced harassment from conspecifics, support in conflicts, and increased feeding efficiency (Nguyen et al., 2009; Kulik et al., 2012; Arseneau et al., 2015; Haunhorst et al., 2017). Subordinates can also gain access to reproduction via dominant reproductive tolerance (sensu Port et al., 2018), as reported for chimpanzees (Bray et al., 2016) and chacma baboons (Henzi et al., 2010). In these species, subordinate mating success is associated with their social investment in the alpha male (Bray et al., 2016; also see Duffy et al., 2007) and subordinates access mating opportunities in return for protection from infanticide and support during challenges by non-resident males (Henzi et al., 2010). 
Reproductive cooperation, defined as the coordinated effort by two or more males to gain reproductive advantage over other males (Díaz-Muñoz et al., 2014), also plays a key role in male reproductive strategies and success. Subordinate olive and yellow baboons can form opportunistic coalitions to interfere with mate guarding by higher ranking males (olive baboons, Papio anubis: Bercovitch, 1988; yellow baboons: Noë and Sluijter, 1990). In the Ngogo chimpanzee community, where male density is exceptionally high, top-ranking males engage in coalitionary mate guarding, which allows them to maximize mating success while minimizing the costs of monopolization (Watts, 1998). In baboons, chimpanzees, and humans, these forms of cooperation emerge as a result of a decrease in individual competitive potential where partner coalitionary choice depends on combined fighting potential (Noë, 1986; Watts, 1998; Benenson et al., 2009).

Similar forms of male reproductive cooperation aimed at communal female defense occur in bottlenose dolphins (Connor et al., 2000; Wiszniewski et al., 2012), horses (Equus ferus caballus: Feh, 1999), as well as other primates characterized by high between-group competition (reviewed in Ostner and Schülke, 2014). In snub-nosed monkeys, unrelated males of different single-male units engage in cooperative defense against satellite males to increase paternity certainty (Rbinopithecus roxellana: Xiang et al., 2014). Kin-based alliances of bachelor males, on the other hand, perform joint offensive actions against units to access reproductive opportunities (Qi et al., 2017). In species where both single- and multi-male groups are present, take-over probabilities are lower for groups with higher numbers of co-resident males (e.g. white-faced capuchins, Cebus capucinus: Fedigan et al., 2004; redfronted lemurs, Eulemur fulvus rufus: Ostner and Kappeler, 2004; geladas: Snyder-Mackler et al., 2012; hamadryas baboons, Papio hamadryas: Chowdhury et al., 2015). In redfronted lemurs the amount of reproduction lost to subordinates is not affected by increasing number of co-resident males (Kappeler and Port, 2008), indicating that in some species the costs of accepting additional males can be negligible (Port et al., 2010). In geladas, where bachelor males constitute a constant threat (Pappano et al., 2012), leader males benefit from leader-follower associations in the form of longer tenure, access to more females, and faster female reproductive rates (Snyder-Mackler et al., 2012). Similarly, in hamadryas baboons the number of associated follower males leads to increased tenure length, number of females, and number of offspring born within the unit (Chowdhury et al., 2015). Despite these similarities two different types of trade-offs seem to operate in the two species. In geladas reproduction is confined to the unit and unit-followers can directly benefit by gaining a small portion of paternities (Snyder-Mackler et al., 2012). In hamadryas baboons, followers may not diminish leader reproduction and, instead, diminish the likelihood of extra-unit paternities (discussed in Chowdhury et al., 2015). Indirect fitness benefits due male kinship and the possibility of future succession may make adopting a follower strategy more beneficial to a non-leader male than a solitary strategy (Chowdhury et al., 2015). 


\subsection{Male bonding and male-male ritualized greeting behavior}

\subsubsection{Male-male bonds and adaptive benefits}

Co-resident males in multi-male groups were thought to be highly competitive and intolerant towards each other due to competition over un-sharable fertilization (van Hooff, 2000). Past studies therefore focused largely on competition, dominance, and aggression (Hill and van Hooff, 1994), but this focus has recently shifted to social interactions beyond simple competition. Several more recent studies found tolerance, cooperation, and affiliation (Boinski, 1994; Silk, 1994; Perry, 1998; Schülke et al., 2010; Berghänel et al., 2011a; Xia et al., 2013; Schoof and Jack, 2014; Teichroeb et al., 2014), with strong male-male bonds described in chimpanzees (Mitani, 2009) as well as Assamese (Kalbitz et al., 2016) and Barbary (Young et al., 2014b) macaques (also reviewed in van Hooff and van Schaik, 1994; Ostner and Schülke, 2014).

Fitness benefits of male sociality and correlations with well-being are now known for several species. Male Assamese macaque investment in strong social bonds is linked to increased coalitionary support, rank ascension and, ultimately, enhanced reproductive success (Schülke et al., 2010). Similarly, male chimpanzees form bonds (Mitani, 2009) and exchange grooming for coalitionary support during conflicts (Watts, 2002). These networks of coalitions are associated with increased rank and improved siring success (Gilby et al., 2013). Male affiliation is linked to coalition formation in other species (Silk, 1994; Perry et al., 2004; Berghänel et al., 2011a; Patzelt et al., 2014; Young et al., 2014b) and coalitions can also affect rank in Barbary macaques (Young et al., 2014c), where affiliative interaction rates during the non-mating season predict coalitions during the following mating season (Berghänel et al., 2011a) and males preferentially recruit closely bonded bystanders (Young et al., 2014b). Studies on Barbary macaques also show that male bonds can buffer against the adverse effects of social and environmental stressors (Young et al., 2014a) and even be linked directly to survival in harsh winter conditions (McFarland and Majolo, 2013; also see Lehmann et al., 2016).

\subsubsection{Male bonding: evolution and mechanisms}

Males can display a broad range of relationship styles when interacting with same-sex conspecifics. The presence of within-group cooperation, affiliation and bonds, however, is not universal (van Hooff and van Schaik, 1994; Ostner and Schülke, 2014). These variations in male sociality led to theoretical frameworks in an attempt to explain the evolution of male bonding (van Hooff and van Schaik, 1994; Ostner and Schülke, 2014). Due to intense male competition over fertilization, it was argued that male bonds should be rare and exclusively occur between kin due to indirect fitness benefits (van Hooff and van Schaik, 1994). Thus, male bonding was thought to be most likely in three scenarios: joint dispersal with kin (natal or secondary), preferential dispersal into groups with resident kin and, especially, male philopatry (van Hooff and van Schaik, 1994; van Hooff, 2000). Behavioral 
biases toward kin can emerge as a result of mother- or father-mediated familiarity, familiarity via age similarity, and phenotypic matching (Widdig, 2007; Smith, 2014). Recent evidence indicates that, while kinship may be a facilitating factor, it is not necessary for the development of cooperation and bonds (Krutzen et al., 2003; Langergraber et al., 2007; Hirsch et al., 2013; Best et al., 2014; Patzelt et al., 2014). Male bonding occurs in species characterized by male dispersal (e.g. bonnet macaques, Macaca radiata: Silk, 1994; Assamese macaques: Schülke et al., 2010; Barbary macaques: Young et al., 2014b) as well as male-philopatric species (e.g. chimpanzees: Mitani, 2009; Guinea baboons: Patzelt et al., 2014). In both types of societies, bonding occurs between both kin and non-kin (Langergraber et al., 2007; Schülke et al., 2010; Patzelt et al., 2014). Findings of low average relatedness within the philopatric sex for large groups (Lukas et al., 2005) further corroborate the limited impact of kinship. While facilitated by inclusive fitness when occurring between kin, male-male bonds and cooperation likely emerge due to direct benefits of mutualistic processes, where all contributors gain more than if they were acting alone (Clutton-Brock, 2002; Boesch et al., 2006a; Clutton-Brock, 2009; Ostner and Schülke, 2014).

The initial precondition for the development of bonds is the presence of stable groups with large number of co-resident males (Ostner and Schülke, 2014). As discussed in previous sections (2.3. and 2.4), multi-male groups emerge as a result of increased between-group competition, which necessitates communal defense against take-overs and extra-group paternity. These associations, however, benefit all group members, can occur despite high levels of within-group competition, and can be loaded with tension (van Hooff, 2000). Although small-scale relationship differentiation (e.g. by age or kin-class) may occur, such group-level cooperation does not require bonds to function (Ostner and Schülke, 2014). Instead, cooperation and affiliation with preferred partners are thought to evolve as a consequence of decreased within-group contest potential (Ostner and Schülke, 2014). In scenarios of medium to low contest potential, where monopolization is less pronounced, male-male coalitions for rank ascension can be crucial to increasing reproductive opportunities (van Schaik et al., 2004, 2006; Ostner and Schülke, 2014). Such coalitions can be risky due to the possibility of retaliation by the high-ranking target (van Schaik et al., 2004, 2006). Increased reproductive benefits from priority of access to females arise over time and are dependent on the long-term maintenance of the newly achieved rank (van Schaik et al., 2004, 2006), which requires reliable and long-term allies consolidated and maintained through strong and stable social bonds (Ostner and Schülke, 2014). Variations in contest potential and their association with coalitionary male strategies, male bonding, and the occurrence of ritualized behavior have been reported across the genus Macaca (Ostner and Schülke, 2014). Additionally, the co-occurrence of rank-changing coalitions and bonds, as well as related benefits (see section 2.5.1), occurs in several species (reviewed in: Ostner and Schülke, 2014; Schülke and Ostner, 2017). 
Other mechanisms may also explain the evolution of social bonds. Individual sociability can result in reduced exposure to predation risk, which benefits individuals in the form of decreased vigilance and increased foraging time (Josephs et al., 2016). Similarly, bond strength enhanced individual responses to recruitment alarm calls and may relate to increased efficiency in cooperative predator defense via better coordination (Micheletta et al., 2012; Kern and Radford, 2016). Sociality may be selected for by promoting efficient cooperation (Melis et al., 2006; Massen et al., 2010), through which individuals can benefit from defense of resources, cofeeding and food sharing, support during aggressive conflicts, and reduced harassment from conspecifics (Nguyen et al., 2009; Berghänel et al., 2011a; Tiddi et al., 2011; Dubuc et al., 2012; Sabbatini et al., 2012; Heesen et al., 2014; Young et al., 2014b; Haunhorst et al., 2017; Samuni et al., 2018b). Although such benefits were discussed largely in relation to female bonds and heterosexual relationships, they may also play a crucial role in favoring the development of male-male social bonds.

\subsubsection{Male-male ritualized greeting behavior}

Group-living requires efficient communication to maintain cohesion and facilitate cooperation (Watson-Jones and Legare, 2016). Ritualized behaviors are stylized, attention-getting and often repetitive (Rappaport, 1979; Rossano, 2012, 2015). They are considered particularly effective in regulating social relationships (Rossano, 2015), as well as promoting coordination and cooperation between individuals conforming to these unambiguous "rule-governed" repertoires (Cullen, 1966; Smuts and Watanabe, 1990). Ritualized interactions are common among males in multi-male groups (e.g. greeting behaviors: Smuts and Watanabe, 1990; Aureli and Schaffner, 2007; De Marco et al., 2014; triadic male-infant interactions: Paul et al., 1996; Kalbitz et al., 2017; Kubenova et al., 2017) and thought to play a role in balancing the trade-offs of male co-residence. These ritualized exchanges should be most frequent in species where male coordination and cooperation must be balanced with the potential consequences of severe male-male aggression (Whitham and Maestripieri, 2003).

Greetings are specific ritualized interactions that are widespread in non-human primates (e.g. Papio spp.: Smuts and Watanabe, 1990; Colmenares et al., 2000; bonobos, Pan paniscus: Hohmann and Fruth, 2000; Macaca spp.: De Marco et al., 2014; Riley et al., 2014) and other mammals (e.g. wild dogs, Lycaon pictus: Estes and Goddard, 1967; Creel and Creel, 1995; spotted hyenas, Crocuta crocuta: East et al., 1993; Smith et al., 2011; African lions: Matoba et al., 2013). These interactions are defined as short exchanges of non-aggressive signals between two individuals (Kutsukake et al., 2006) comprising a uni- or bi-directional exchange of cohesive or affiliative signals (Peláez, 1982). These signals are species-specific behavioral patterns that vary in complexity and intensity, ranging from touches and embraces (e.g. spider monkeys, Ateles geoffroyi: Aureli and Schaffner, 2007; black-horned capuchin monkeys, Cebus nigritus: Lynch Alfaro, 2008) to genital manipulation and same-sex mounting (e.g. Guinea baboons: Whitham and Maestripieri, 2003; spotted hyenas: Smith et al., 2011; Tonkean 
macaques, Macaca Tonkeana: De Marco et al., 2014). Notwithstanding the label "greetings", they are not limited to departures and reunions. Instead, this term is used for a broad variety of ritualized interactions that occur in diverse contexts and have different functions (De Marco et al., 2014). Greetings are hypothesized to function in promoting coordination and group cohesion (Estes and Goddard, 1967; Creel and Creel, 1995; Lynch Alfaro, 2008), buffer tension during tense contexts like fusion events (East et al., 1993; Aureli and Schaffner, 2007; Lynch Alfaro, 2008) and competition over resources (Colmenares et al., 2000; Hohmann and Fruth, 2000), and assessing dominant status (East et al., 1993; Hohmann and Fruth, 2000) and/or relationship quality (Wang and Milton, 2003; Whitham and Maestripieri, 2003; De Marco et al., 2014). In some species, greetings also function to reinforce and maintain social bonds and promote cooperation (Smuts and Watanabe, 1990; Smith et al., 2011; De Marco et al., 2014). In sum, greetings can serve similar functions in different species (e.g. Aureli and Schaffner, 2007; Lynch Alfaro, 2008) but also be used differently within the same species (e.g. spotted hyenas: East et al., 1993; Smith et al., 2011) and between populations of the same species (e.g. mantled howlers, Alouatta palliata: Wang and Milton, 2003; Dias et al., 2008).

The genus Papio and some species of the genus Macaca are characterized by particularly intense and highly ritualized male-male greeting repertoires that involve high-risk behaviors like mounting and genital manipulations (e.g. olive baboons: Smuts and Watanabe, 1990; crested macaques, Macaca nigra: Reed et al., 1997; Guinea baboons: Whitham and Maestripieri, 2003; moor macaques, Macaca maura: Riley et al., 2014). The occurrence, context, and function of greetings vary considerably between species in the genus Papio. In chacma baboons, where males avoid each other and affiliation and coalition are non-existent, greetings are virtually absent (Henzi and Barrett, 2005; Henzi et al., 2008; but see Saayman, 1971). In hamadryas baboons, where males maintain affiliative relationships and cooperate in group defense (Colmenares et al., 2007; Swedell, 2011), greetings are quasi-aggressive and occur mainly in competitive contexts allowing males to negotiate and buffer tension (Hall and DeVore, 1965; Kummer et al., 1974, 1985; Abegglen, 1984; Colmenares, 1990, 1991a, 1991b; Colmenares et al., 2000). In this species, greeting initiation and asymmetries are associated with male social status (Hall and DeVore, 1965; Colmenares, 1990, 1991b). Instead, studies in olive baboons, which present opportunistic coalitions but no affiliative relationships (Busse, 1986; Bercovitch, 1988), and Guinea baboons, which display spatial tolerance, affiliative relationships and cooperation (Fischer et al., 2017), characterized greetings as affiliative signals (Smuts and Watanabe, 1990; Whitham and Maestripieri, 2003). While in olive baboons associations between greeting roles and dominance rank depend on age and residence status (Smuts and Watanabe, 1990), in Guinea baboons male rank distance does not predict greeting roles (Whitham and Maestripieri, 2003). In these species, greetings allow males to assess relationship quality independent of context and play a role in bond maintenance (Whitham and Maestripieri, 2003) and promoting cooperation (Smuts and Watanabe, 1990). 
Similar to baboons, the occurrence of ritualized behavior is associated with male affiliation and cooperation in macaques (Ostner and Schülke, 2014; De Marco, 2017), with a lack of ritualized interactions in intolerant male rhesus (Macaca mulatta) and Japanese macaques (Macaca fuscata), and presence of greetings and/or triadic male-infant interactions in species with male tolerance and coalitions (e.g. Silk, 1994; De Marco et al., 2014; Riley et al., 2014; Kalbitz et al., 2017; Kubenova et al., 2017; discussed in De Marco, 2017). As reported for baboons, the function of these interactions varies considerably. In some species these ritualized interactions function to assess relationship quality (De Marco et al., 2014) and maintain social bonds (Kalbitz et al., 2017) between males. These findings suggest that the function of greetings, and possibly other ritualized interactions, varies between species and that, in some cases, this can be associated with the tenor of male-male relationships and level of male tolerance and cooperation (Henzi et al., 2008; De Marco et al., 2014; De Marco, 2017).

Much of the research on greetings, however, is based on data collected in captivity (e.g. Whitham and Maestripieri, 2003; De Marco et al., 2014), wild provisioned groups (Riley et al., 2014), few individuals (Riley et al., 2014), or mixed species and their hybrids (e.g. Colmenares, 1990, 1991a, 1991b). More long-term systematic comparative field studies are required for a deeper understanding of how these ritualized interactions regulate male-male relationships in different social systems.

\subsection{Guinea baboons as a model species}

So far in this thesis I introduced the wide range of relationship styles displayed by co-resident males, emphasized how male-male associations are beneficial in the context of high inter-group competition, and highlighted how low to medium within-group contest potential can lead to the development of male-male cooperation and social bonds. I also elucidated how variations in the occurrence and role of ritualized behaviors may depend on the presence of cooperation and tolerance. The diverse social systems of the genus Papio, characterized by various levels of male-male competition, cooperation, and tolerance, are an ideal model for examining the presence and role of social bonds and ritualized greetings. Guinea baboons constitute an ideal test case to address these questions as they present high levels of spatial tolerance, coalition formation, and low competition (Kalbitzer et al., 2015; Fischer et al., 2017). In this thesis I examine the dynamics and fitness benefits of male-male sociality in wild Guinea baboons with a focus on the features and functions of ritualized greeting behavior and the patterns and adaptive benefits of male-male associations and bonds.

Guinea baboons live in nested multilevel societies (Patzelt et al., 2014). At the core of the society are "units" composed of a primary male, associated females, immatures, and sometimes secondary males. Several units form stable "parties" and two to three parties regularly aggregate into "gangs" with overlapping home ranges (Patzelt et al., 2014; Goffe et al., 2016; Fischer et al., 2017). Genetic data shows evidence for female-biased dispersal and males are predominantly philopatric (Kopp et al., 2015) though sparse male transfers may occur (Dal Pesco \& Faraut, personal observation; also dis- 
cussed in Patzelt, 2013). Although male-male average relatedness is higher within than between gangs, it is not significantly different between parties of the same gang (Patzelt et al., 2014). Adult males present high levels of spatial tolerance, form affiliative relationships, and support each other in coalitions even after establishing mating relationships (Patzelt et al., 2014). Males of the same party spend a higher proportion of time in spatial association and exchange significantly higher frequencies of affiliative interactions and coalitionary support (Patzelt et al., 2014). Males often exchange intense greeting interactions but their function is still disputed (Fischer et al., 2017). Compared to other species of the genus Papio, adult males show very low aggression rates, no clear dominance hierarchy, and rare injuries (Kalbitzer et al., 2015). The interplay between male philopatry and affiliative relationships is proposed as possible explanation for this low level of competition (Jolly, 2009; Patzelt et al., 2014). A previous study that focused on male-male relationships at the gang level, however, found that affiliation, greetings, and coalitions occurred regardless of kinship (Patzelt et al., 2014). While further corroboration is needed, these findings suggest kinship plays a limited role in male-male relationships (Fischer et al., 2017). Observations of early male-male association development in a captive population (Boese, 1975) do suggest that male long-term relationships could represent a fundamental element in Guinea baboon social dynamics.

Male tolerance extends to relationships with females and to relationships between females and secondary males (Goffe et al., 2016). Primary males form stable associations with one to six females and these units may include secondary males (Goffe et al., 2016). Females interact significantly more with their primaries and their reproductive state has little influence on the frequency of these interactions (Goffe et al., 2016). Relationships between females and associated secondary males are weaker. Secondary males have social but, usually, no sexual access to females with $98.6 \%$ of copulations occurring between females and their primary (Goffe et al., 2016). Preliminary investigations show femalefemale associations and rates of affiliative and aggressive interactions are significantly greater between females of the same unit (Goffe, 2016), a common feature of other polygyn-monandrous systems, like in hamadryas baboons (Swedell, 2002). Compared to hamadryas baboons, take-over attempts are rare and males don't display "conditioning" aggression or herding toward females (Goffe, 2016; Goffe et al., 2016). Guinea baboon females exhibit high spatial freedom, can respond to male aggression with counter-aggression and female coalitions, and play an active role in intersexual relationship maintenance (Goffe et al., 2016). This suggests Guinea baboon females may exhibit a level of female choice (Goffe et al., 2016). The relaxed tenor of Guinea baboon relationships and high level of tolerance sets them apart from other species within the genus Papio (Fischer et al., 2017).

\subsection{Project aims}

In study 1 (chapter 3) I explore the features and function of ritualized greeting behavior in wild Guinea baboons and provide the first systematic analysis in a wild population. I first explore greeting 
occurrence at the various levels of this multilevel society, accounting for the differences in partner availability between animals that belong to the inner-most versus outer layers of the society. Then I turn to my core question and investigate the function of within-party male-male ritualized greetings. To determine if this behavior is simply a literal greeting, I test whether they occur randomly simply when individuals are in close proximity (Fraser and Plowman, 2007). Next, I test if greetings are used to assess relationship quality between spatially tolerant and affiliative partners (Smuts and Watanabe, 1990; Whitham and Maestripieri, 2003), or whether greetings buffer tension between males. For the latter I analyze three scenarios: male dyads with higher levels of aggression, temporal association with aggressive episodes, and the presence of receptive females (reviewed in Colmenares et al., 2000). As these two hypotheses are not mutually exclusive, I also investigate if greetings serve multiple functions depending on the dyadic relationship type (i.e. affiliative/non-affiliative dyads). Lastly, I review the features of Guinea baboon greetings and compare them with other species in this genus.

In study 2 (chapter 4) I explore the features and adaptive benefits of male-male sociality in wild Guinea baboons and focus on within-party male-male relationships. I first examine the main features of male-male sociality, namely the patterns of association of primary and their secondary males and male-male affiliative relationships more generally. I examine if Guinea baboons form social bonds by testing the two most common criteria (Silk, 2002): whether affiliative relationships are differentiated within the party and whether they are stable over time. This is followed by an investigation of the role of kinship in male-male sociality. While a previous analysis on a smaller sample at the gang level revealed no correlation between relatedness and affiliation, I return to this question with a larger and more detailed dataset at the party level. I also apply this dataset to an investigation of the link between coalitionary support and social bonds, as well as a reassessment of the male dominance hierarchy. I then analyzed male reproductive skew in our population for the first time to test whether malemale sociality enhances male reproductive success in the form of number of associated females and sired offspring. 


\title{
3. Study I
}

\section{Greetings in Male Guinea Baboons and the Function of Rituals in Complex Social Groups}

\author{
Federica Dal Pesco ${ }^{1,2,3}$ \& Julia Fischer,1,2,3 \\ ${ }^{1}$ Cognitive Ethology Laboratory, German Primate Center, Göttingen \\ 2Leibniz ScienceCampus Primate Cognition, Göttingen \\ 3Department for Primate Cognition, Georg-August-University Göttingen, Göttingen
}

Journal of Human Evolution: 125: 87-98.

DOI: $10.1016 /$ j.jhevol.2018.10.007 


\section{Author Contributions}

Federica Dal Pesco and Julia Fischer designed the study; Federica Dal Pesco collected, extracted and analyzed the data; Federica Dal Pesco and Julia Fischer wrote the manuscript; Federica Dal Pesco and Julia Fischer discussed the results and commented on the manuscript. 
The manuscript of this section was previously published (non-open access) in the Journal of Human Evolution (volume 125, year 2018) and needed to be excluded from the published text of this dissertation for copyright reasons.

It can be found at the following Elsevier link:

https://www.sciencedirect.com/science/article/abs/pii/S0047248418302021 


\title{
4. Study II
}

\section{Fitness Benefits of Male-Male Sociality in Wild Guinea Baboons (Papio papio)}

\author{
Federica Dal Pesco ${ }^{1,2,3}$, Franziska Trede ${ }^{1,3}$, \\ Dietmar Zinner ${ }^{1} \&$ Julia Fischer ${ }^{1,2,3}$ \\ ${ }^{1}$ Cognitive Ethology Laboratory, German Primate Center, Göttingen \\ ${ }^{2}$ Leibniz ScienceCampus Primate Cognition, Göttingen \\ 3Department for Primate Cognition, Georg-August-University Göttingen, Göttingen
}

In preparation 


\section{Author Contributions}

Federica Dal Pesco and Julia Fischer designed the study; Federica Dal Pesco collected, extracted, and analyzed the data; Franziska Trede conducted the genetic laboratory analysis; Dietmar Zinner supervised the genetic laboratory analysis; Franziska Trede and Federica Dal Pesco conducted the genetic relatedness and paternity analyses; Federica Dal Pesco and Julia Fischer wrote the manuscript; Federi-

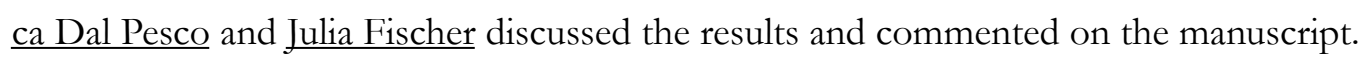




\subsection{Abstract}

Male-male bonds and multi-male associations may confer substantial fitness benefits. The adaptive value of these relationships is often attributed to coalitionary support, which aids in rank ascension and female defense. Male Guinea baboons form bonds with other males, but they do not reveal a clear rank hierarchy, which allowed us to assess the adaptive value of male-male sociality in a tolerant society. We therefore investigated whether males with strong bonds are more attractive to females. Using social behavior, genetic relatedness, and paternity data for 24 males, we here show that malemale affiliative relationships were differentiated and stable over time. Strongly bonded males revealed a higher degree of relatedness on average and were more likely to support each other in coalitions. Contrary to our predictions, male-male sociality had no positive effect on the number of associated females or sired offspring. Instead, males with stronger bonds had significantly lower numbers of associated females. Apparently, time constraints affect male investment in different types of relationships.

Keywords: Coalitionary support; Kinship; Male-male relationships; Papio papio; Reproductive benefits; Social bonds

\subsection{Introduction}

Male-male cooperation (Grinnell et al., 1995; Feh, 1999; Snyder-Mackler et al., 2012; Connor and Krützen, 2015), affiliation (Silk, 1994; Perry, 1998; Schoof and Jack, 2014; Teichroeb et al., 2014) and social bonds (Mitani, 2009; Young et al., 2014b; Kalbitz et al., 2016) have been described between both kin and non-kin in numerous mammalian species. Adaptive benefits of these associations have been widely reported in the form of reduced influence of environmental stressors (Young et al., 2014a), increased survival (McFarland and Majolo, 2013; Lehmann et al., 2016), and enhanced reproductive success (Schülke et al., 2010; Snyder-Mackler et al., 2012; Wiszniewski et al., 2012; Gilby et al., 2013; Chowdhury et al., 2015). In particular, the effects on male reproductive success are often attributed to within-group coalitionary support and communal group defense. Investment in social bonds for coalitionary support and coalition networks are associated with rank ascension and reproductive success in Assamese macaques (Macaca assamensis: Schülke et al., 2010) and chimpanzees (Pan troglodytes: Gilby et al., 2013). In several mammal species, multi-male associations benefit dominant males by aiding in female defense, which can result in longer tenure and/or increased number of females and offspring (Feh, 1999; Fedigan et al., 2004; Ostner and Kappeler, 2004; Snyder-Mackler et al., 2012; Wiszniewski et al., 2012; Chowdhury et al., 2015). Female choice can also be influential with males that invest in bonds with females and provide protection, assistance in rearing young, and support in conflicts also gaining higher reproductive success (Kulik et al., 2012; Massen and Vries, 2012; 
Ostner et al., 2013; Arseneau et al., 2015; Rosenbaum et al., 2018). Females may additionally prefer males with more and stronger intrasexual relationships due to their greater average sociability.

The presence of male-male affiliation and cooperation, combined with the relatively high level of female leverage in mate choice (reviewed in Fischer et al., 2017), renders Guinea baboons (Papio papio) a valuable model to determine how the advantages conferred by male-male sociality play out in a highly tolerant multilevel system. Nested within Guinea baboon societies are "units" composed of a "primary" male, 1-6 associated females, immatures, and often "secondary" males that generally interact socially but not sexually with the females (Goffe et al., 2016). Several units make up a "party" and 2-3 parties regularly aggregate into "gangs" with overlapping home ranges (Patzelt et al., 2014). Males are predominately philopatric, maintain high spatial tolerance, exchange affiliation, and support each other in coalitions (Patzelt et al., 2014). Compared to other baboon species, adult males show low rates of aggression, no clear dominance hierarchy, and take-over attempts are extremely rare (Kalbitzer et al., 2015; Goffe et al., 2016). Females have unusually high levels of spatial freedom and play an active role in the formation and maintenance of inter-sexual relationships (Goffe et al., 2016).

Building on the findings of our initial analyses (Patzelt et al., 2014; Goffe et al., 2016), we here examine the adaptive benefits of male-male sociality in wild Guinea baboons. We first tested if males fulfill two of the most commonly used criteria for identifying "strong bonds" (Silk, 2002), namely whether affiliative relationships within parties are differentiated and stable over time. We defined secondary males as non-primary males that were most frequently in spatial association with a primary male and interacted with its females (see Chowdhury et al., 2015). Although our previous analysis based on a small sample revealed no correlation between affiliation and relatedness, we returned to this question here with a larger dataset and predicted that kinship affects bonds and association with secondary males. We also re-evaluated the link between coalitionary support and social bonds and predicted that dyads with stronger bonds were more likely to cooperate during conflicts than dyads with weaker bonds. Finally, we turned to our core question and tested whether male bond strength, number of friends, and number of secondary males were linked to enhanced reproductive success in the form of increased numbers of associated females and sired offspring.

\subsection{Materials and methods}

\subsubsection{Field site and study subjects}

Data for this study were collected over 19 months, from April 2014 to October 2015, at the Centre de Recherche de Primatologie (CRP) Simenti field station in the Niokolo-Koba National Park (PNNK), Senegal (described in Maciej et al., 2013). The Simenti Guinea baboon community comprises over 400 individuals, including five habituated parties in two gangs, the Mare and the Simenti gang. We selected two parties with the highest numbers of adolescent and adult males as our study groups (par- 
ty 9 from the Mare gang and party 6 from the Simenti gang). Party was used as the group unit in this study because males display higher proportions of time in spatial association (within $20 \mathrm{~m}$ ) and higher rates of affiliation, greeting, and coalition at the party level (Patzelt et al., 2014; Fischer et al., 2017; Dal Pesco and Fischer, 2018). Therefore, our analyses focused on within-party dyads. Party size and composition varied during the study period due to maturation, mortality, and dispersal/migration, with an average of 44.5 individuals in party 6 (range 2014-2015: 40-48, average adult sex ratio: 88.89) and 44.5 individuals in party 9 (range 2014-2015: 38-51, average adult sex ratio: 47.83; see supplementary table S4.1a). We performed continuous behavioral observations of all adult and adolescent (i.e. large juvenile, small and large subadult) males. Each month, two independent observers differentiated developmental stages and assessed age categories using physical markers (see supplementary S4.2a and S4.2b). Males were introduced as focal subjects when they reached the status "large juvenile male". Three males disappeared during the study period (likely due to predation), while three males that transitioned to large juvenile status were included later in the study. We observed a total of 24 individuals (average: 21.5 , range: $21-22$; party $6, \mathrm{n}=14$; party $9, \mathrm{n}=10$ ) during the study period with an average observation time per subject of 17 months (range: 3-19). Long-term data from two subsequent years (2016 and 2017) were supplemented to investigate male relationship stability (see supplementary tables S4.1a, S4.1b and S4.3 for detailed information about data collection between 2014 and 2017).

\subsubsection{Data collection}

We conducted behavioral observations during morning (6:30-12:30) and afternoon (15:00-18:00) sessions for a total of 410 observation days (884 contact hours for party 9 and 941 contact hours for party 6). All data were collected on Samsung Note 2 handhelds using electronic forms developed for our long-term data collection in the Pendragon 7.2 software (Pendragon Software Corporation, USA). We recorded census information concerning demographic changes (presence, birth, absence or death), health status, and female reproductive state on a daily basis (Goffe et al., 2016). Aggression, displace/supplant, avoidance, unprovoked submission, coalitionary support, copulation and grooming were recorded ad libitum. We conducted focal follows (Altmann, 1974) of 20 mins and balanced between subjects and morning and afternoon sessions, for an average of seven monthly protocols per individual and a total focal time of $956 \mathrm{~h}$ (total number of focal protocols=2961). Protocols included recordings of continuous focal animal activity (i.e. moving, feeding, resting, and socializing) and all occurrences of social behaviors such as approach (within $1 \mathrm{~m}$ ), retreat, grooming, contact-sit, and greeting. All grooming and contact-sit bout durations were recorded to the nearest second. Scan sampling (Altmann, 1974) was used before and after each focal protocol to record all focal male neighbors within $10 \mathrm{~cm}, 1 \mathrm{~m}, 5 \mathrm{~m}$ and $10 \mathrm{~m}$ (total number of proximity scans=5911). 


\subsubsection{Data analyses}

Statistical analyses (including figure preparation) were conducted using the $\mathrm{R}$ environment (version 3.4.4; R Development Core Team, 2018) in the RStudio interface (version 1.1.456; RStudioTeam, 2018). Specific functions and packages are mentioned in each sub-section. All males $(n=24)$ were included in all analyses, except those of reproductive success (i.e. reproductive skew, number of associated females, sired offspring) where only large subadult and adult males $(n=20)$ were included. Note that no association with females or siring of offspring were observed in younger age categories (unpublished data). Males were included as large subadult if recorded in this category for more than half of the observation time in a specific year.

\subsubsection{Male social bonds: strength and stability}

We investigated if male Guinea baboons form social bonds by assessing whether male-male affiliative relationships were differentiated and stable over time (Silk, 2002). We measured the strength of dyadic affiliative relationships by computing the dyadic composite sociality index (hereafter DSI; Silk et al., 2006b, 2006a, 2010a, 2010b, 2013). Ranging from 0 to infinity, this index measures the deviation of affiliative behavior of a given dyad compared to all other dyads in the same group. The mean DSI value is 1 , lower values represent affiliative relationships weaker than average, and higher values indicate stronger than average relationships. We calculated the DSI on a yearly basis (January to December) for each male-male dyad based on the method by Silk et al. (2006a, 2006b). The only exception was the analysis of average dyadic relatedness and male sociality (see section 4.3.10 for details), for which the DSI was computed for the whole study duration (i.e. 2014 and 2015 combined). The following behaviors were included in our index calculation: grooming frequency and duration, contactsit frequency and duration, and frequency of within $1 \mathrm{~m}$ approaches (see supplementary table S4.4a). Note that only approaches that were not followed by social behavior (positive or negative) within 10 sec were considered in the DSI calculation. Based on Silk et al. (2006a, 2006b) we assessed the correlation between behavioral components in our index. We used the "cor.test" function in the "stats" package (R Development Core Team, 2018) to perform a Kendall's tau correlation test and calculate the tau correlation coefficient and respective p-value. All behavioral components included in the composite index were positively correlated (see supplementary table S4.4b). Individual social bond strength was calculated as the sum of a male's top three DSI values (Silk et al., 2003, 2010b). In addition, based on the methodology by McFarland and colleagues (2013; 2015, 2017), the number of strong bonds (hereafter friends) per male was based on the number of higher than average DSI values.

We calculated male-male relationship stability using the Partner Stability Index each year over a 4-year period (PSI) (Silk et al., 2006a, 2013). This index measures variation in individual partner preference based on each individual's top partners across several time periods. The PSI ranges from 0 to 1 , 
where 1 is the highest stability value, for individuals with the same top partners over all periods, and 0 is the lowest stability value, for individuals that changed top partners in every period. All males present for at least two consecutive periods (total=23) were included in this analysis (no gaps occurred between observation periods of individual males). For some males, no single third top partner could be identified. We therefore calculated all PSI values using the top two partners. To test if our observed preference patterns were different from those expected by chance, we compared the observed PSI values for each individual male against mean expected PSI values for random partner choice calculated from 10.000 permutations (Silk et al., 2012; Kalbitz et al., 2016) using an exact Wilcoxon signed rank test ("wilcoxon.test" function in the "stats" package R Development Core Team, 2018; two-sided, paired, confidence level $=0.95)$.

\subsubsection{Unit composition and associated secondary males}

Data on female-male associations were recorded daily. Female unit transfers were recorded and verified using copulations, grooming bouts, contact-sit bouts, greetings, and aggression events from focal and ad libitum data (Dal Pesco and Fischer, 2018). As shown in a previous study, females in a unit exchanged significantly higher rates of interactions with the primary male (Goffe et al., 2016). Therefore, we used interaction occurrence (see Dal Pesco and Fischer, 2018) to assess female-male associations and unit composition.

Following methodologies established for hamadryads baboons (Chowdhury et al., 2015; p. 503), we defined secondary males as non-primary adolescent/adult males who were most frequently within 1 $\mathrm{m}$ proximity of a primary male and who interacted at significantly higher rates and spent a significantly higher proportion of time within $1 \mathrm{~m}$ of the primary male's females compared to other co-resident males (see supplementary appendix 4.1, related supplementary tables, and supplementary figures S4.1 and S4.2). All associated males exchanged affiliative interactions (i.e. grooming and contact-sit; associated males: mean $\pm \mathrm{SD}=27.03 \pm 26.55$; non-associated males: mean $\pm \mathrm{SD}=0.82 \pm 1.69$; see supplementary appendix 4.1). Note that in this species, secondary males can be associated with multiple units. To control for the influence of female transfers and demographic changes on secondary male associations, we calculated the number of secondary males per unit as a yearly average weighted by the duration of the association in days.

\subsubsection{Male dominance assessment}

Ad libitum and focal data on male-male aggression (i.e. threats, lunges, chases, physical fights), displace/supplant, avoidance, and unprovoked submission were used to assess the dominance hierarchy among adolescent and adult males of the same party. Dyadic decided interactions were used to compile a winner/loser matrix to determine dominance relationships for each studied party. We excluded any aggressive interaction that followed one or more polyadic interactions within the same aggressive 
event (polyadic event). When several interactions per dyad occurred within the same aggression event, only the highest intensity interaction was considered. As previous investigations in our population indicated adult males do not present a clear and significant linear hierarchy (Patzelt et al., 2014; Kalbitzer et al., 2015), we used the "aniDom” package (Farine and Sánchez-Tójar, 2018) to calculate the randomized Elo-rating scores for males of each party (Sánchez-Tójar et al., 2018). We chose this method as it performed best for both intermediate and low hierarchy steepness in a data simulation study (Sánchez-Tójar et al., 2018). This methodological approach also enables the assessment of hierarchy steepness and uncertainty independent of group size and sampling effort. We assessed the degree of hierarchy orderliness using the triangular transitivity index (Shizuka and McDonald, 2012). Compared to others, this index is not influenced by dataset sparseness or variation in group size (Shizuka and McDonald, 2012). For methodological details and functions used in this analysis see supplementary appendix 4.2a.

\subsubsection{Genetic relatedness and paternity analyses}

We collected fecal samples of all adolescent/adult males $(n=24)$ and subadult/adult females $(n=27)$ of our two study parties for a total of 51 individuals. All offspring born in 2014 and 2015 were identified for a total of 24 infants. Of these, we were able to sample 19 offspring for paternity analysis while the 5 remaining offspring were deceased before sampling could occur. We characterized genetic variation by assessing the individual allele variation on 24 polymorphic autosomal microsatellite markers (see supplementary appendix 4.3 and 4.4 and supplementary table S4.5). We used individual genotypes to run male-male dyadic relatedness analyses and paternity analyses. We calculated descriptive statistics for all 24 markers (including FIS, expected and observed heterozygosity) and tested for Hardy-Weinberg equilibrium (HWE) and presence of null alleles in both genotype datasets (see supplementary appendix 4.3 and supplementary tables S4.6a and S4.6b). One locus was excluded and a total of 23 loci were included in the following analyses. We estimated dyadic relatedness for all 24 adolescent and adult males belonging to parties 6 and 9 (total within-party dyads: 134) using the $\mathrm{R}$ package "related" (Pew et al., 2015; also see Wang, 2011) and chose the Wang relatedness estimator (Wang, 2002) (see supplementary appendix 4.3). We estimated paternity for 19 sampled offspring born during 2014 and 2015 (see table supplementary table S4.10) and assigned paternity using a likelihood approach with the software Cervus (version 3.0.7; Kalinowski et al., 2007). A male was considered to have sired an offspring when he was assigned as the most likely father, had 0 to maximum 1 mismatched alleles, and the confidence level for the assignment was more than 95\% ("strict" criterion). See supplementary appendix 4.3 for analysis details.

\subsubsection{Analyses of reproductive skew}

To investigate male reproductive success and skew at the party level we used all offspring born in 2014 and 2015 with assigned paternity ( $\mathrm{n}=18$; one offspring was fathered by a male of another party; 
see supplementary appendix 4.3) to calculate the Binomial Skew Index (B index) (Nonacs, 2000) with the SKEW CALCULATOR 2013 (Nonacs, 2003). Contrary to other skew indices, the B index can account for group demographic changes (Nonacs, 2003) allowing us to include demographic information regarding the number of days each male was present in our studied parties. This index ranges from -1 to 1 with positive values indicating a skew greater than expected for a random distribution of reproductive benefits and negative values indicating a more equitable sharing of benefits. The software uses a simulation approach (10000 simulations were calculated) to calculate $95 \%$ confidence intervals, the maximum possible B value (i.e. "B-monopoly": reproduction monopolized by one individual) and the minimum possible B value (i.e. "B-equal": equal sharing among all individuals). The observed skew is not different from chance if the confidence intervals include zero. Moreover, if the confidence interval includes the B-equal or the B-monopoly value, then equal distributions of benefits or total monopoly by one individual cannot be excluded, respectively.

Moreover, to investigate primary male reproductive success at the unit level, we calculated the percentage of offspring sired by the primary male of each unit. Note that during the study period no mothers transferred to another unit between conception and birth.

\subsubsection{Statistical analyses and modeling}

We ran generalized linear mixed models using the R package "lme4" (Bates et al., 2015). Detailed information about sample size, data standardization/transformation, model structure, full null model comparison, and diagnostics (assumptions, collinearity, overdispersion, model stability) can be found in the supplementary appendix 4.5 and in each model table (supplementary tables S4.11-S4.16). Pvalues were obtained from the likelihood ratio test performed with the R function "drop1" with argument "test" set to "chisq" (Barr et al., 2013). Effect sizes were calculated with the "summ" function of the "jtools" R package (version 1.1.1; Jacob and Long, 2018).

\subsubsection{The role of kinship in male sociality}

To investigate if kinship played a role in male sociality we tested whether average dyadic relatedness was significantly higher for friends versus non-friends and primary and their secondary males versus all other dyads. Male-male dyads were characterized as friends if their DSI (calculated over both years, 2014 and 2015) was above average (i.e. above 1) and as primary/secondary if they were counted as primary and associated secondary male at least once during this period. We ran two generalized linear mixed models with a Gaussian error structure (Baayen, 2008) with dyadic relatedness estimates (wang estimator) as the response and relationship type 1 (friends and non-friends) and type 2 (primary/secondary and non-primary/secondary) as the main predictor, respectively. In both models we included party membership as fixed control factors and male identities as random intercepts. Note 
that in this analysis the two study years were considered as a single study period due to the fact that relatedness estimates (our response) are constant over time.

\subsubsection{Male sociality and coalitionary support}

Coalitionary support was scored every time two or more individuals simultaneously directed aggression toward a common target that could be a single male or another male-male coalition. All occurrences of aggression events and coalitionary support were recorded ad libitum. We scored male-male coalitions within each aggression event with the following criteria: if two individuals A and B supported each other in several aggressive interactions within the same aggression event this was scored as one coalitionary support (AB); if $A, B$ and $C$ supported each other during the same aggressive interaction this was scored as three coalitionary supports ( $\mathrm{AB}, \mathrm{BC}, \mathrm{AC})$; if during the same event, $\mathrm{A}$ was supported only by $\mathrm{B}$ during an aggressive interaction and only by $\mathrm{C}$ during a following aggressive interaction, this was scored as two coalitionary supports ( $\mathrm{AB}$ and $\mathrm{AC})$.

To investigate whether male-male dyads with stronger bonds were more likely to support each other in coalitions we ran a generalized linear mixed model with Poisson error structure and log link function (McCullagh and Nelder, 1989) where dyadic coalitionary support frequency per year was the count response and yearly dyadic DSI was the main predictor of interest. Due to the very low rate of aggression (Patzelt et al., 2014; Kalbitzer et al., 2015), all occurrences of male-male coalitionary support recorded both from focal and ad libitum data were included in this analysis. To control for different observation durations per party and dyad we included the log-transformed contact time in hours as an offset (McCullagh and Nelder, 1989). Note that this was calculated using the total time spent working with the studied parties during each daily working session and for each male-male dyad to account for demographic changes. We included year and party membership as fixed control factors, and male identities and dyad identity as random intercepts. The following random slope components were also included: year and dyadic DSI within male identities.

\subsubsection{Male sociality and reproductive success}

To investigate if male sociality enhanced male reproductive success in the form of associated females and offspring we calculated the number of associated females and sired offspring per male per year. To control for female transfers and demographic changes we calculated the number of associated females per unit as a yearly average weighted by the duration of the association in days. Our main predictors of male sociality were yearly male bond strength, number of friends, and weighted average number of associated secondary males. As by definition only primary males can have secondary males, we ran two separate models with different datasets and predictors of interest for each measure of reproductive success. A first model included all large subadult and adult males $(n=20)$ and both bond strength and number of friends as predictors. A second model only included males that were 
primary at least once during the study period $(n=17)$ and the predictor of interest weighted average number of associated secondary males. We ran two generalized linear mixed models with Gaussian error structure (Baayen, 2008) where weighted average number of associated females was the response and two generalized linear mixed models with Poisson error structure and log link function (McCullagh and Nelder, 1989) where number of sired offspring per year was the count response. In the first two models the gaussian response was log transformed (see details in supplementary table S4.14a and S4.14b). In all four models we included year $(2014,2015)$ as fixed control factor, while male identity was included as random intercept. To control for the effect of group size and available partners on the number of social bonds (Silk et al., 2018), we included male partner availability (i.e. total number of males present within each party per year) as a fixed control variable in all four models. We did not include party membership due to multicollinearity issues with male partner availability.

\subsubsection{Post-hoc analysis: time males spent socializing with other males by number of associated females}

In light of the results of our analysis, we performed a post-hoc investigation of the effect number of associated females had on the proportion of time males spent socializing (i.e. grooming plus contactsit) with other males. We ran a generalized linear mixed model with a Gaussian error structure (Bayen, 2008) with the proportion of time males spent socializing with other males as response and number of associated females as predictor of interest. The response was square-root transformed (see details in supplementary table S4.16). Party membership was included as fixed control factor and male identity as random intercept.

\subsection{Results}

\subsubsection{Male-male sociality}

Male-male affiliative relationships were differentiated and stable over time. The distribution of the dyadic composite sociality index (DSI) was highly skewed, indicating that relationships were differentiated within the party (see figure 4.1 and supplementary figure S4.3). The DSI for the entire study period (see supplementary tables S4.7a and S4.7b for yearly values) ranged from 0.0 to 13.4 with a mean of 1 and a median of 0.1 . Only $22.4 \%$ of dyads (30/134 dyads) had a DSI above group average, and the top $10 \%$ of relationships had a DSI above 3.54. To illustrate the variation in behaviors in relation to DSI, average approach rates per hour were 0.32 for a DSI of $0.5,0.73$ for a DSI of 1 , and 1.62 for a DSI of 5 . Average male bond strength (calculated as the sum of a male's top three DSI values) was 10.04 for party $6(\mathrm{SD}=4.25$; range $=0.97-16.89 ; \mathrm{n}=14)$ and 8.26 for party $9(\mathrm{SD}=5.01$; range $=1.71-15.13 ; n=10$ ). The average number of friends per male (calculated as the number of higher than average DSI values) was 3.29 for party $6(\mathrm{SD}=2.13$; range $=0-6 ; \mathrm{n}=14)$ and 1.40 for party $9(\mathrm{SD}=0.84$; range $=0-3 ; \mathrm{n}=10)$. 


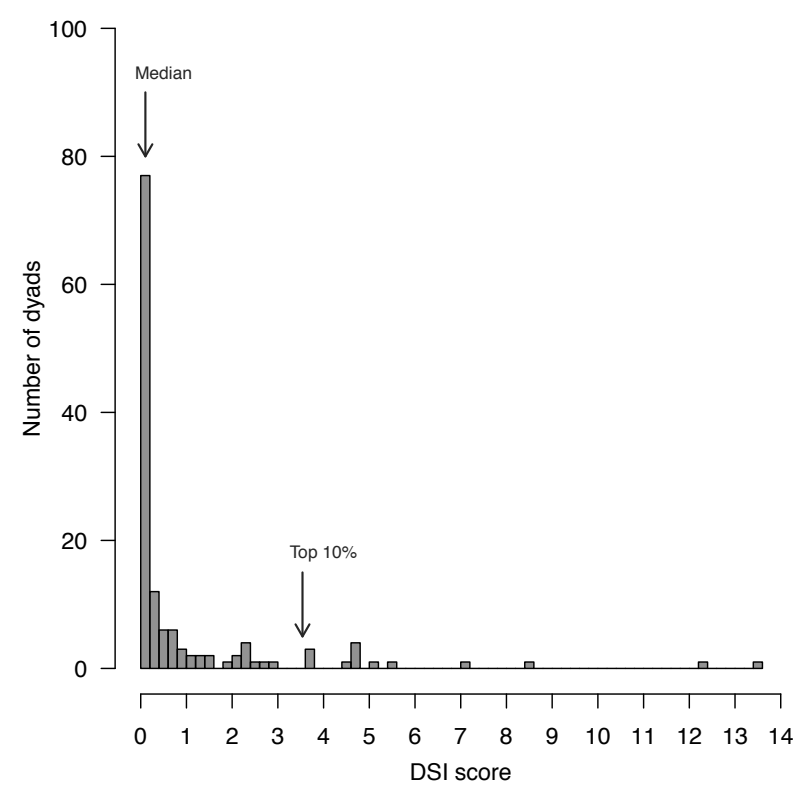

Figure 4.1 Distribution of the dyadic composite sociality index (DSI) calculated for the entire study period (2014 and 2015) for all male-male within-party dyads. This index measures the strength of dyadic affiliative social relationships compared to the average of the group. DSI values from both parties are pooled (mean $=1$, median $=0.102$, range $=$ 0.000-13.437, also see supplementary table $\mathrm{S} 4.7 \mathrm{a}$ and supplementary figure S4.3).

$(\mathrm{SD}=1.47$; range $=0.00-4.08)$. The weighted average number of associated secondary males per primary male was 2.56 (range $=0.00-4.08 ; n=14)$ for party 6 and $0.62($ range $=0.00-1.43 ; n=10)$ for party 9 (see supplementary table S4.7b for yearly values). Conversely, $66.7 \%$ of secondary males interacted with females of and were associated with multiple units (average number of units $2.60 \pm 1.45 \mathrm{SD}$; range=1-5). With only one exception, all secondary males were either adolescents or late prime/old males (also see Goffe et al., 2016). Most primary males maintained their status from the acquisition of their first female to late prime/old age (unpublished data, also see Goffe et al., 2016). Strong affiliative relationships between males were not restricted to the unit level, with $39.9 \%$ of friends consisting of non-primary/secondary dyads (i.e. primary/primary and non-primary/non-primary dyads). During the study period, all 24 males were either primary or secondary and no unaffiliated male was present in either party. Past observations, however, indicate rare occurrences of unaffiliated males (Goffe et al., 2016).

\subsubsection{Male dominance assessment}

Out of 1026 within-party male-male aggressive interactions, only 19.1\% (196 interactions) could be used to assess dominance because the others were non-decided (42.7\% of 1026$)$, non-dyadic $(59.7 \%$ of 1026) or comprised repeated interactions within the same bout of aggression. These 196 interactions were used in combination with 209 displace/supplant, avoidance, and unprovoked submission interactions, for a total of 405 interactions, to determine the rank hierarchy. Both parties showed a 

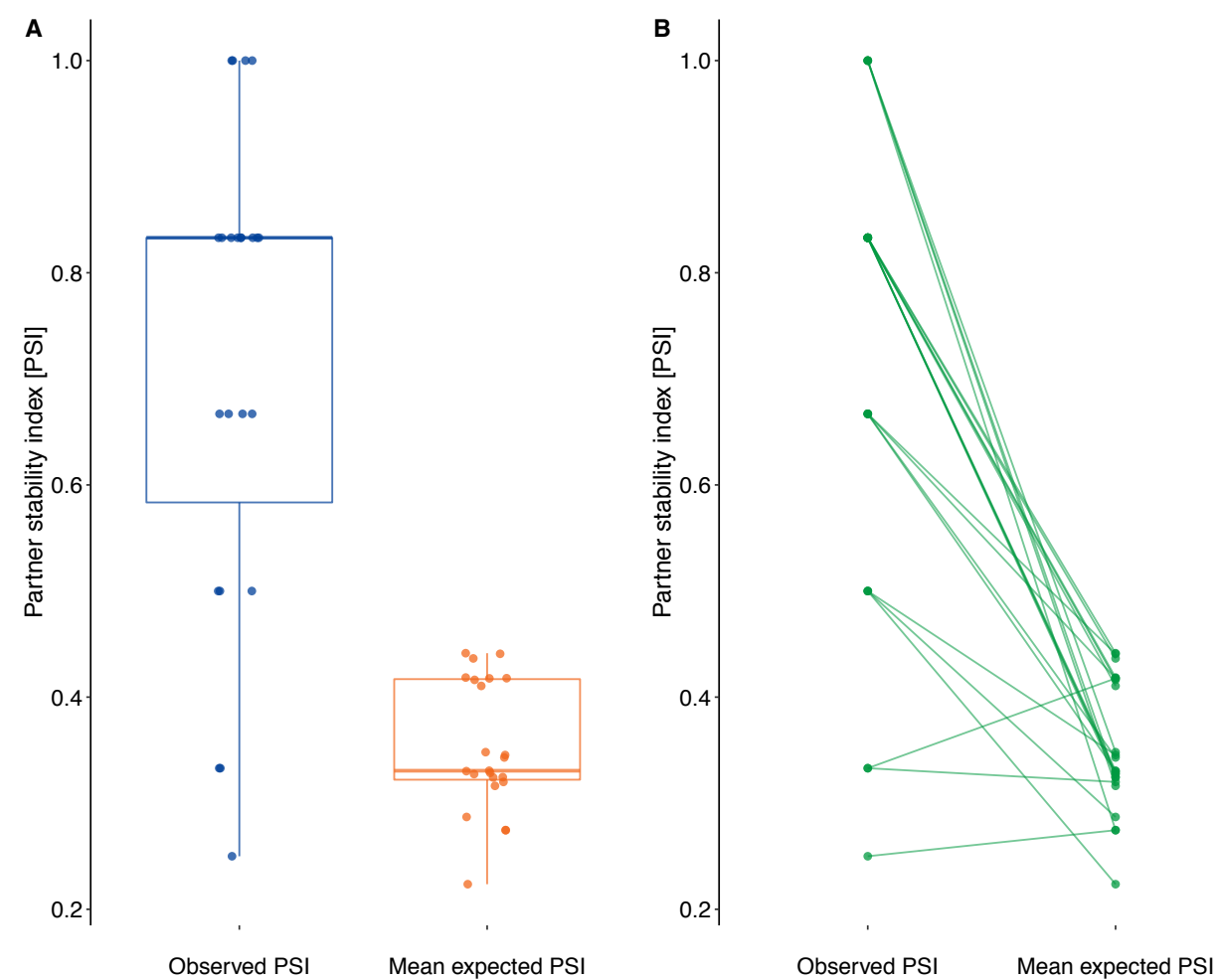

Figure 4.2 Male partner stability indices (PSI) over a 4-year period ( $\mathrm{n}=23$ ). Observed PSI values are compared against mean expected PSI for random partner choice calculated from 10.000 permutations. Observed PSI values were significantly higher than mean permuted PSI values. A) the median, lower and upper quartiles $(25 \%$ and $75 \%)$ and the range excluding outliers (vertical line). B) changes between observed and expected PSI values for each individual male.

hierarchy of intermediate/low steepness with high variation in randomized Elo-rating scores and great overlap between the $95 \%$ score range for most males. Hierarchies showed high transitivity, indicating high levels of orderliness. Yet, due to the very low rate of aggression (Patzelt et al., 2014; Kalbitzer et al., 2015) and usable proportion of interactions, our attempts to assess dominance yielded highly uncertain estimates. Therefore, dominance could not be included as a predictor in our analyses (see supplementary appendix 4.2b and 4.2c supplementary tables S4.8a and S4.8b, and supplementary figures S4.4 and S4.5).

\subsubsection{Analyses of reproductive skew}

At the party level, reproductive skew (measured using the Binomial Skew Index) was low and the share of reproductive benefit (i.e. sired offspring, $n=19$ ) was not significantly different from chance in both party 6 ( $B$ index obs. $=0.028$; $C I=-0.112-0.246 ; \mathrm{p}=0.273$ ) and party 9 (B index obs. $=-0.043$; $\mathrm{CI}=-0.087-0.072 ; \mathrm{p}=0.856$ ). Moreover, in both parties equal distributions of reproduction could not be excluded (see supplementary table S4.9). 89.5\% of offspring $(n=17)$ were sired by the primary male, suggesting a high mate fidelity in this species. The remaining $10.5 \%(n=2)$ of offspring were sired by a subadult male belonging to the same party and an adult male belonging to a different party of the same gang, respectively, indicating that sneaky copulations can even occur across parties (see supplementary appendix 4.3 and supplementary table S4.10 for paternity analysis details). 


\subsubsection{The role of kinship in male sociality}

Relatedness estimates for male-male withinparty dyads averaged $0.06(\mathrm{SD} \pm 0.24)$ and ranged from -0.56 to 0.69 (median=0.05). As predicted, kinship played a significant role in male sociality. Male friends had significantly higher average relatedness than non-friends (estimate $\pm \mathrm{SE}=-0.210 \pm 0.045, \mathrm{p}<0.001$, figure 4.3, see supplementary table S4.11a). Note that dyadic relatedness ranges overlapped to a great extent (friends: range $=-0.31-0.69$; nonfriends: range $=-0.56-0.57$ ), suggesting that bonds were neither exclusively restricted to kin nor did kinship always guarantee stronger social bonds. The same pattern was found for primary males and their associated secondary males, where primary/secondary dyads had significantly higher average relatedness than non-primary/secondary dyads (estimate $\pm \mathrm{SE}=$ $-0.175 \pm 0.045, \quad p<0.001$, primary/secondary:

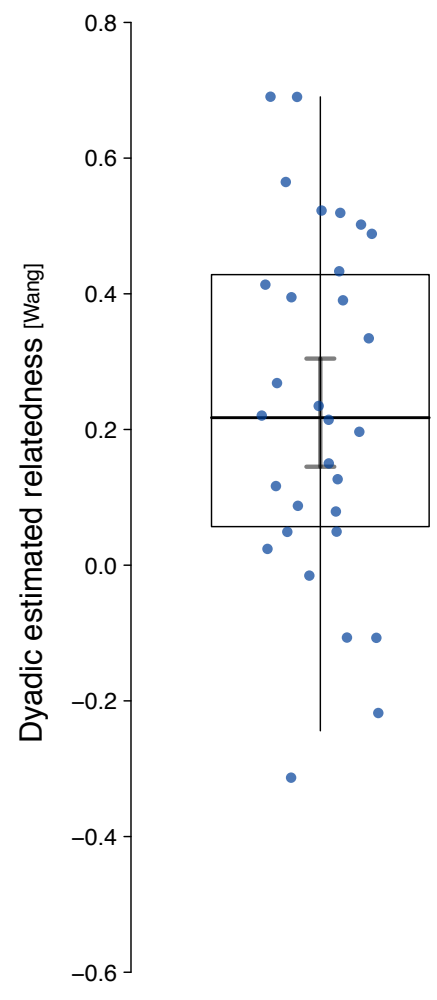

Friends

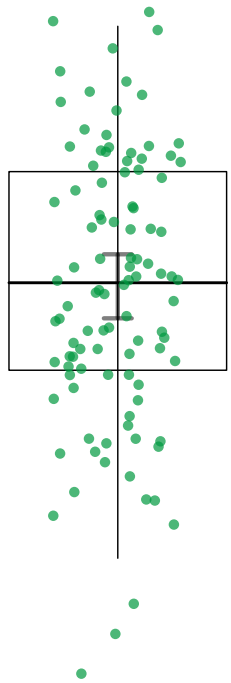

Non-friends

Figure 4.3 Average dyadic male-male relatedness estimates (wang) by relationship type 1 (friend versus non-friend dyads). Friend dyads had significantly higher average relatedness estimates than non-friend dyads. Points depict within-party dyads (134 dyads, 24 males). Boxplots depict the median, the lower and upper quartiles $(25 \%$ and $75 \%)$, and the range excluding outliers (vertical line). Short horizontal whiskers depict the bootstrapped $95 \%$ confidence intervals (party manually dummy coded and centered). range $=-0.31-0.69$; non-primary/secondary: range $=-0.56-0.57$; supplementary figure S4.6, see supplementary table S4.11b).

\subsubsection{Male sociality and coalitionary support}

A total of 362 dyadic coalitions were recorded between adolescent/adult males during the study duration (both from focal and ad libitum data). Coalitions regularly included several males and conflicts often involved coalitions targeting other coalitions. Dyads supported each other on average 2.70 times $(\mathrm{SD}=5.40$; range $=0-36)$ in ca. 1800 contact hours with an average rate per hour and dyad of 0.002 $(\mathrm{SD}=0.003$; range $=0.000-0.020)$. Coalitionary support was recorded both within and between units, with only $42.0 \%$ of coalitions taking place between primary and their secondary males. Dyads with stronger bonds (higher DSI values) were significantly more likely to support each other in coalitions (higher dyadic coalition rates; estimate $\pm \mathrm{SE}=0.765 \pm 0.127, \mathrm{p}<0.001$, figure 4.4 , see supplementary table S4.12). 


\subsubsection{Male sociality and reproductive success}

The weighted average number of associated females per large subadult/adult male was 0.88 for party $6(\mathrm{SD}=0.89$; range $=0.00-2.68 ; \mathrm{n}=14)$ and 1.69 for party $9(\mathrm{SD}=1.32$; range $=0.00-3.15$; $\mathrm{n}=10)$. The full model including the two predictors of interest (bond strength and number of friends) accounted for significantly more variance compared to the null model (full null model comparison: $\chi 2=7.163, \mathrm{df}=2, \mathrm{p}=0.028)$. While number of friends was not associated with the number of associated females (estimate $\pm \mathrm{SE}=$ $-0.008 \pm 0.070, \mathrm{p}=0.911)$, males with stronger bonds had significantly fewer females (estimate \pm $\mathrm{SE}=-0.193 \pm 0.071, \mathrm{p}=0.010$; see figure 4.5). Results remained unchanged if the strength of all bonds (instead of male's top three DSI values) was used to calculate male bond strength (data not shown). We additionally examined whether the weighted average number of associated sec-

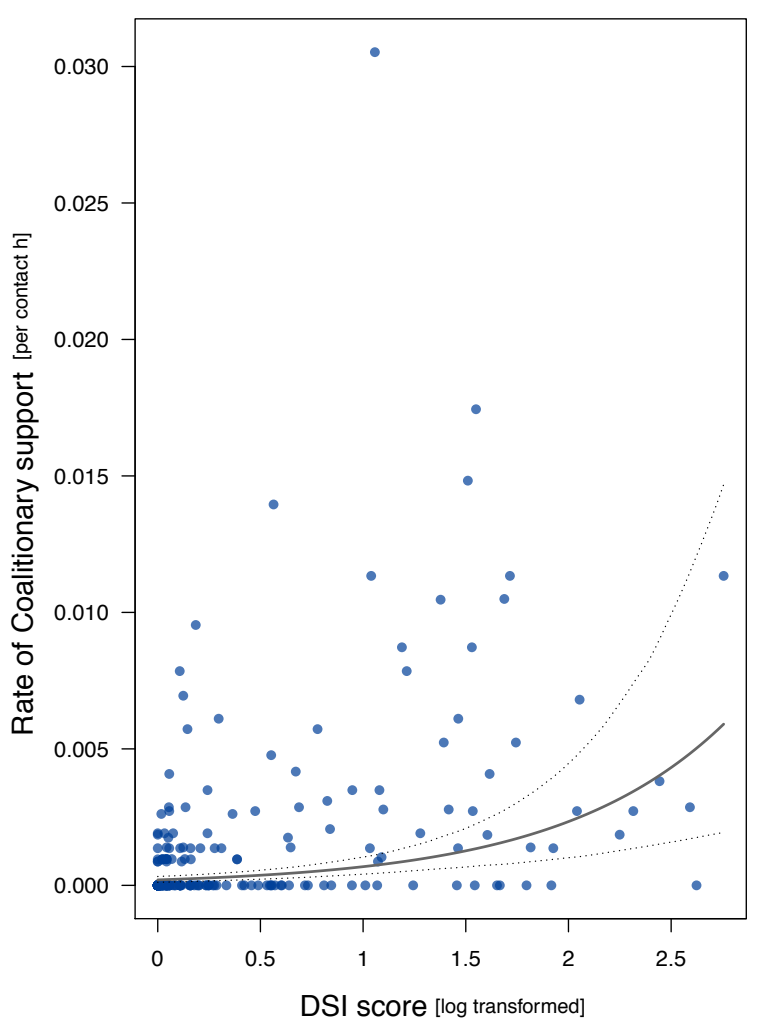

Figure 4.4 Effect of male-male dyadic bond strength (DSI values or scores) on rate of coalitionary support (calculated from ad libitum and focal data and corrected by contact time in hours). Dyads with stronger bonds were significantly more likely to support each other in coalitions. Points depict each dyad twice (2014 and 2015). DSI was log transformed and subsequently standardized to a mean of zero and a standard deviation of one (original means and standard deviations in supplementary table S4.12). The solid line depicts the fitted model and dashed lines depict the bootstrapped $95 \%$ confidence intervals (party and year manually dummy coded and centered). ondary males predicted the number of females. There was no association between the weighted average number of secondary males and the number of females (estimate $\pm \mathrm{SE}=0.002 \pm 0.140, \mathrm{p}=0.989$; see supplementary tables $\mathrm{S} 4.13 \mathrm{a}$ and $\mathrm{S} 4.13 \mathrm{~b})$.

The average number of sired offspring per large subadult/adult male was 0.73 for party $6(\mathrm{SD}=1.01$; range $=0-3 ; n=14)$ and 1.11 for party $9(S D=0.93$; range $=0-2 ; n=10)$. The full model with the two predictors of interest (bond strength and number of friends) did not account for significantly more variance compared to the null model (full null model comparison: $\chi^{2}=1.658, \mathrm{df}=2, \mathrm{p}=0.437$ ). Considering the very low effect size (Pseudo- $\mathrm{R}^{2}$ (total) $=0.138$ ), this is unlikely to be a type II error. Thus, neither the strength of male social bonds nor the number of male friends was associated with the number of sired offspring (bond strength: estimate $\pm \mathrm{SE}=-0.283 \pm 0.320$; friends: estimate $\pm \mathrm{SE}=$ $0.427 \pm 0.341)$. The additional model examining the weighted average number of associated secondary males found that this measure of male sociality was also not associated with the number of sired offspring (estimate $\pm \mathrm{SE}=-0.019 \pm 0.338, \mathrm{p}=0.955$; see supplementary tables $\mathrm{S} 4.14 \mathrm{a}$ and $\mathrm{S} 4.14 \mathrm{~b}$ ). 


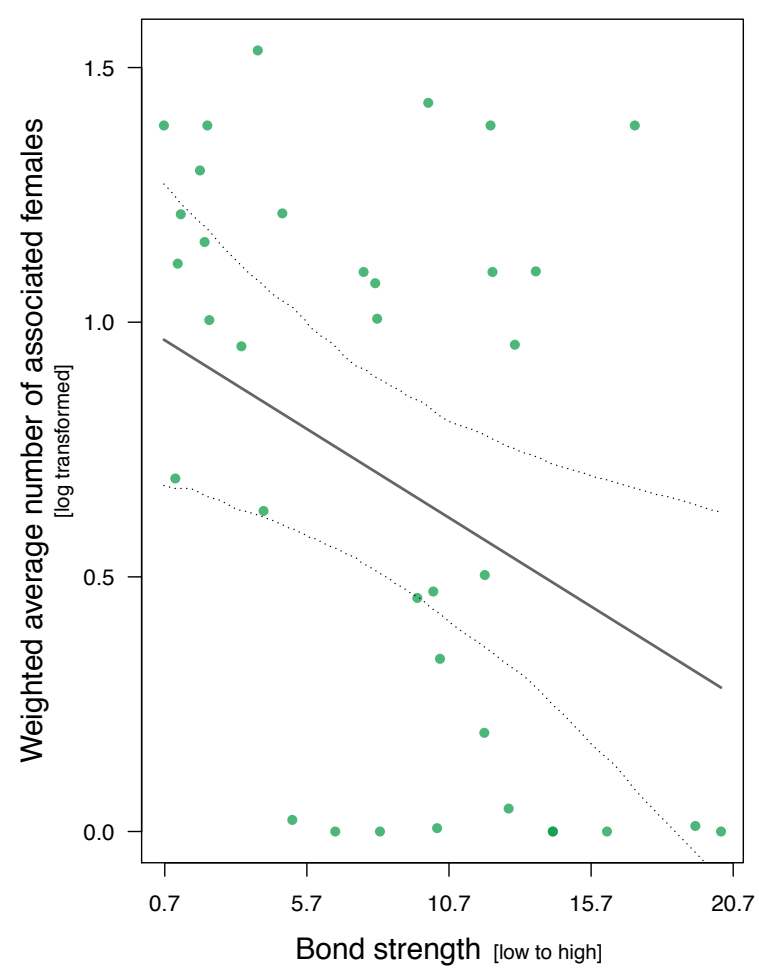

Figure 4.5 Effect of male bond strength (calculated as the sum of a male's top three DSI values) on average number of associated females (calculated as average weighted by the duration of the association in days). Males with stronger bonds were found to have significantly fewer females. Points depict each dyad twice (2014 and 2015). The response was $\log$ transformed and the covariates standardized to a mean of zero and a standard deviation of one (original means and standard deviations in supplementary table S4.13a). The solid line depicts the fitted model and dashed lines depict the bootstrapped 95\% confidence intervals (year manually dummy coded and centered and male partner availability standardized to a mean of zero and a standard deviation of one).

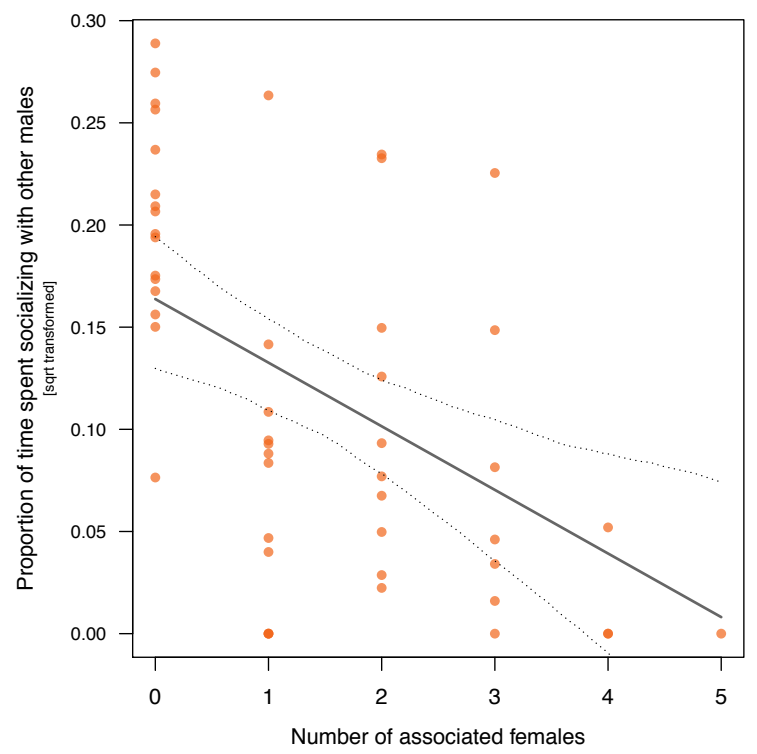

Figure 4.6 Effect of number of associated females on proportion of time spent socializing with other males (i.e. grooming plus contact-sit). Males with higher numbers of associated females spent significantly lower proportions of time socializing with other males. The response was square-root transformed (original means and standard deviations in supplementary table S4.15). The solid line depicts the fitted model and dashed lines depict the bootstrapped $95 \%$ confidence intervals (party manually dummy coded and centered).

\subsubsection{Post-hoc analysis: effect of number of associated females on time spent socializing with other males}

As male bond strength was linked with lower numbers of associated females, we performed a post-hoc analysis to explore the effect the number of associated females has on time males spent socializing (i.e. grooming plus contact-sit) with other males. Males with a higher numbers of associated females spent a significantly lower proportion of time socializing with other males (estimate $\pm \mathrm{SE}=-0.042 \pm 0.011, \mathrm{p}=0.001$; see figure 4.6 and supplementary table S4.15). The average proportion of time spent socializing with other males decreased from $0.04 \quad(\mathrm{SD}=0.02$; range $=0.01-0.08)$ for males having no associated females to 0.01 ( $\mathrm{SD}=0.02$; range $=0.00-0.07)$ for males with one associated female, while the average proportion of time spent socializing for males with four to five females was only 0.001 $(\mathrm{SD}=0.001 ;$ range $=0.000-0.003)$.

\subsection{Discussion}

Guinea baboon males maintain highly differentiated relationships with other males. The differentiated and stable nature of male-male affiliative relationships indicates that male Guinea baboons form strong social bonds. Primary males may develop strong bonds with other primary males as well as with non-primary males associated to their unit called "secondary males". Strong bonds were also observed between non-primary males. Affiliative relationships and coalitionary support between males were not restricted to the unit level, with $39.9 \%$ of strong bonds ("friendships") and $58 \%$ of coalitions not recorded between 
primary males and their associated secondary males. Notably, bachelor groups are not characteristic of this species, unaffiliated males (termed "solitary" in hamadryas baboons) occur rarely, and secondary males can be associated with multiple units at the same time. These results set Guinea baboon males apart from hamadryas baboons and geladas. Hamadryas baboon leader males occasionally groom with follower/solitary males and do not affiliate with other leaders (Schreier and Swedell, 2009), while male-male affiliation in geladas is restricted to bachelor group males (Pappano, 2013) or to rare interactions between leaders and their followers (Dunbar and Dunbar, 1975). Moreover, most primary Guinea baboon males had associated secondary males $(76.5 \%$ versus $55.4 \%$ in hamadryas and $33.3 \%$ in gelada) and most of these units had more than one secondary male $(61.5 \%$ versus $38.9 \%$ in hamadryas; mean $\mathrm{n}$. of secondaries per unit: 1.65 versus 0.80 in hamadryas) (see SnyderMackler et al., 2012; Chowdhury et al., 2015). Contrary to hamadryas baboons, where follower males are mainly associated with a particular unit and further associations are short-term and may not extend to females (Chowdhury et al., 2015), Guinea baboon secondary males have multiple associations that can last several years and involve interactions with primary males as well as associated females. These results, in combination with the rarity of takeover attempts, female spatial freedom, and an active female role in intersexual relationships (Goffe et al., 2016), highlight the fluidity and uniqueness of the Guinea baboon social system.

Within-party male sociality was linked to kinship, which was likely a major mechanism in the evolution of male-male social bonds in this species. Guinea baboon males biased their behavior toward relatives, with significantly higher average relatedness between friends, as well as between primary and their secondary males. However, kinship is not the only driving force as it was associated with some bonds but not others. Similar patterns were reported for chimpanzees (Langergraber et al., 2007; Mitani, 2009) and bottlenose dolphins (Tursiops of. australis: Diaz-Aguirre et al., 2018), where the indirect benefit of male affiliation and cooperation co-occurred with direct benefits obtained through mutualistic and reciprocal relationships. The basis of kin discrimination in this species is, however, still unknown, so that we cannot exclude that mechanisms such as familiarity might play a more central role in shaping patterns of male social preferences. While age proximity and association with a common parent may act as a mechanism for kin discrimination (Smith, 2014) at the unit level due to the high likelihood of being sired by the same father, it is unlikely to be a very reliable indicator of relatedness overall due to the low reproductive skew at the party level. This ambiguity is compounded by the reported female transfers between all social levels, females transferring with their dependent offspring, and lack of evidence for infanticide (Goffe et al., 2016), leading to a scenario where relatedness and familiarity do not exactly mirror each other. Therefore, if familiarity is the major mechanism of kin discrimination in Guinea baboons, unit composition changes during early development might result in less overlap between male relatedness and sociality. Indeed, reports from a captive population of Guinea baboons suggested that male-male associations may emerge early in life (Boese, 1975). 
Multi-male associations can confer important benefits in form of communal defense (Feh, 1999) and lower likelihood of takeovers (Fedigan et al., 2004; Ostner and Kappeler, 2004). In geladas and hamadryas baboons, associated males benefit leader males through increased tenure length, number of females or number of offspring (Theropithecus gelada: Snyder-Mackler et al., 2012; Papio hamadryas: Chowdhury et al., 2015). Male cooperative unit defense plays a key role due to the high level of intergroup competition characterized by unit takeovers followed by female acquisition and often infanticide (Swedell and Tesfaye, 2003; Beehner and Bergman, 2008; Pappano et al., 2012; Snyder-Mackler et al., 2012; Swedell et al., 2014; Pines et al., 2015). Associated males can also benefit from a small proportion of reproduction, which, as seen in geladas, can be restricted to the unit (Snyder-Mackler et al., 2012). In contrast, our results showed evidence for occasional extra-unit paternities and, particularly, that number of associated secondary males in Guinea baboons was not linked to higher number of females or sired offspring. This is likely the case because $76.5 \%$ of males have secondary males, $61.5 \%$ of these have more than one secondary male and, most importantly, secondary males have stable associations with multiple units, all of which leaves little room for variation in reproductive success. Secondary males may not be as crucial to unit defense as in other species, as takeovers are rare (Goffe et al., 2016; Fischer et al., 2017) and male competition is low (Patzelt et al., 2014; Kalbitzer et al., 2015; Fischer et al., 2017). This would also mean that Guinea baboon females, unlike geladas or hamadryas, do not gain much benefit from preferring males with alliances, and may instead prefer males that provide higher levels of affiliation and spatial freedom (Goffe, 2016) or, as seen in mountain gorilla (Rosenbaum et al., 2018), that care more for offspring.

At the party level, Guinea baboon males cooperated via coalitionary support both within and between units. Males with stronger bonds supported each other more often during agonistic events, which is consistent with findings in several macaque species (Silk, 1994; Schülke et al., 2010; Berghänel et al., 2011a; Young et al., 2014b), where sociality predicted levels of male cooperation. In red howler monkeys (Alouatta seniculus) male coalitions were key in establishing and maintaining tenure within troops (Pope, 1990), while coalitionary support in Assamese macaques was linked to future dominance success and, in turn, to enhanced reproductive success (Schülke et al., 2010). In Guinea baboons, however, competition and rank play only a marginal role (Patzelt et al., 2014; Kalbitzer et al., 2015; Fischer et al., 2017; Dal Pesco and Fischer, 2018; see supplementary appendix 4.2c) and number of friends and bond strength were not positively linked to either number of females or number of sired offspring. Compared to macaques (Schülke et al., 2010; Berghänel et al., 2011a), rates of coalitionary support in Guinea baboons are surprisingly low (Guinea baboons: 0.002/hr; Assamese macaques: $0.11 / \mathrm{hr}$; Barbary macaques, Macaca sylvanus: $0.01-0.21 / \mathrm{hr}$ ), mirroring the low aggression rate (Kalbitzer et al., 2015). Our results indicate that sociality is not, as reported in other species, linked to increased short-term reproductive success. What is still unknown is why Guinea baboon males engage in coalitions and whether they confer benefits in the long-term. 
Contrary to our predictions, males with stronger bonds had significantly lower numbers of associated females. Partner preference was stable over time, but male sociality appeared to be influenced by time budget, requiring numbers of associated females to be balanced out by less time invested in malemale relationships. As reproduction is non-seasonal (Boese, 1973) and primary males sired $89.5 \%$ of offspring within their unit, stable and long-term associations with females are likely of high value, and, as male interactions with females are independent of reproductive state (Goffe et al., 2016), this investment needs to be continuous and year-round. In another species presenting male-male bonds, the Barbary macaque, time investment in male-male relationships is affected by female reproductive state, with grooming interaction rates between males dropping in the mating season (Berghänel et al., 2011a, 2011b; Young et al., 2014b). Our results show that this effect is not only restricted to seasonally breeding species. In experimental studies on hamadryas baboons, strong female preferences for the leader male have been associated with lower likelihood of takeover attempts (Bachmann and Kummer, 1980), indicating the crucial role of pair bond relationship quality for some species. In Guinea baboons, rates of aggression towards females are less than half of those in geladas and hamadryas baboons (Goffe et al., 2016) and repeated herding behavior (i.e. "conditioning") has not been reported (Goffe, 2016), suggesting that inter-sexual dynamics are not simply established by coercion. The fluidity of Guinea baboon society, where females interact with secondary males, have high levels of spatial freedom, and respond to male aggression with occasional counter-aggression and female-female coalitions (Goffe, 2016; Goffe et al., 2016), may in fact require higher levels of male investment in the form of affiliation and vigilance.

Our study show that male-male sociality did not enhance short-term male reproductive success in Guinea baboons opening the door to new research regarding both the influence of female-male bonds in male social dynamics and the possible long-term benefits of male sociality. While female unit size affected male-male relationship investment in the form of reduced time spent socializing, male sociality may vary more generally in relation to male reproductive status, with non-primary males investing more in same-sex relationships. This is supported by observations in our population where males that lost primary status engaged in frequent affiliation with males that were previously close to their unit (unpublished data). What is still unclear is if male-male relationships are indeed maintained over the life time and how this is achieved. One possibility is the use of the most intense forms of male-male ritualized greetings. A previous study found that these intense ritualized interactions occurred most often between dyads with higher affiliation rates (Dal Pesco and Fischer, 2018), while studies on macaques suggest that ritualized interactions might be most efficient in maintaining bonds due to time budget constraints (Kalbitz et al., 2017). While bonds with females are crucial, due to their direct fitness benefits, male-male bonds may benefit males in the form of early unit establishment, delayed loss of primary status, and increased tolerance and support. Overall, social relationships may also combat the detrimental effects of social isolation (Hawkley et al., 2012; Holt-Lunstad 
et al., 2015) regardless of the sex of the social partner. Long-term data on this population will be fundamental in investigating whether male sociality has long-term effects on male fitness in the form of tenure length and number of females/offspring over the entire reproductive lifespan.

\subsection{Acknowledgments}

We would like to thank the Diréction des Parcs Nationaux (DPN) and Ministère de l'Environnement et de la Protéction de la Nature (MEPN) de la République du Sénégal for permission to work in the Niokolo Koba National Park. We particularly thank the former conservateur of the park Colonel Ousmane Kane for his cooperation and logistical support; all the staff and field assistants of the CRP Simenti, in particular Mustapha Faye, Armél Louis Nyafouna, Elhadji Dansokho, Lamine Diedhiou, Moustapha Dieng and Touradou Sonko for their support in the field. We thank Matthis Drolet, Lauriane Faraut, Mathieu Cantat, Fransziska Wegdell and Eréna Dupont for their assistance in the field. We also thank Roger Mundry for his precious statistical support and Matthis Drolet for invaluable comments on the manuscript. This research was supported by the Deutsche Forschungsgemeinschaft (DFG, German Research Foundation) - Project number 254142454 / GRK 2070.

\subsection{Compliance with ethical standard}

Approval and research permission was granted by the DPN and the MEPN de la République du Sénégal (research permit numbers: 0383/24/03/2009; 0373/10/3/2012). Research was conducted within the regulations set by Senegalese agencies as well as by the Animal Care Committee at the German Primate Center.

\subsection{Ethical approval}

All applicable international, national, and/or institutional guidelines for the care and use of animals were followed. All procedures performed in studies involving animals were in accordance with the ethical standards of the institution or practice at which the studies were conducted.

\subsection{Data availability}

The data that support the findings of this study are available from the corresponding author upon reasonable request. 


\subsection{Supplements}

\section{Appendix 4.1 Methodological assessment of associated secondary males}

To assess the number of secondary males associated with a primary male's unit we considered both male-male and male-female perspective. For the male-male perspective, we used spatial association to determine associated and non-associated males for each primary male. For the male-female perspective, we tested if associated males interacted/associated at significantly higher rates with the females of the primary male in contrast to non-associated males. This analysis was based on the methodologies and definitions developed for hamadryas baboons (Chowdhury et al., 2015; p. 503).

\section{The male perspective: male-male association}

To classify male-male dyadic association strength we conducted a change point analysis to detect discontinuities in the distribution of the rate of male-male approaches (within 1m). All approaches recorded between males of the same party during the study period were included in this analysis. We ran this analysis using the R package "changepoint" (version 2.2.2, Killick et al., 2016; R version 3.4.4 and RStudio version 1.1.456) and the function "cpt.meanvar" that investigates both mean and variance changes (method was set to "PELT", penalty to "MBIC", and test statistic to "Exponential").

Three different change point locations were identified in the distribution (see supplementary figure S4.1). We considered the largest change point (change point at dyad number 34; approach rate $=0.402$ ) as our association threshold and divided male-male dyads dependent on if their approach rate was above or below this threshold to label them as associated or non-associated, respectively. Associated males exchanged an average of 27.03 affiliative interactions (i.e. grooming and contact-sit) during the study period $(\mathrm{SD}=26.55$; median $=22.50$; range $=3-139)$. This indicate that all associated males exchanged affiliative interactions. Non-associated males exchanged an average of 0.82 affiliative interactions $(\mathrm{SD}=1.69$; median $=0.00$; range $=0-9)$.

The female perspective: behavioral patterns of associated versus non-associated males and the primary male's associated females

To take into account unit composition changes due to female transfers, we focused this analysis on all periods during which unit composition was stable and split our main dataset accordingly. Furthermore, we restricted our analysis exclusively to stable periods with a duration of at least 6 weeks (i.e. no female transfers from or to the unit recorded for at least 42 days). We identified a total of 34 stable periods with an average duration of 180.32 days $(S D=119.06$; range $=47-579)$. These periods include 15 different primary males with an average of 2.27 ( $\mathrm{SD}=1.1$; range=1-4) stable periods per male.

For every stable period of each unit we counted all occurrences of copulations, grooming bouts, contact-sit bouts, greetings, and aggression events between the females associated with that unit and 
every other male of the party (excluding the primary male of the unit). These behavioral counts were calculated from male focal protocol data and ad libitum data separately. Applying the same method on our proximity scan data, we also calculated the number of times every other male of the party and all females associated with the unit spent in $1 \mathrm{~m}$ proximity for every stable period. To correct these counts for sampling effort we calculated the focal observation duration, the contact time with the party, and the total number of proximity scans for every stable period. Note that these were calculated taking into account all male demographic changes that occurred during every stable period.

To test whether associated males interacted with the primary male's females at a significantly higher rate we ran a generalized linear mixed model with Poisson error structure and log link function (McCullagh and Nelder, 1989) where male-male association type (associated vs. non-associated male) was the main predictor of interest. We ran three different models with different count responses: the count of ad libitum interactions with the primary male's females (model 1a); the count of focal interactions with the primary male's females (model 1b); the number of times recorded in $1 \mathrm{~m}$ proximity to the primary male's females (model 1c). To control for sampling effort, we included the log-transformed focal duration (in h), the contact time with the party (in h) and the number of proximity scans as offsets (McCullagh and Nelder, 1989) in models 1a, 1b and 1c, respectively. We included party membership and number of females associated with the unit (in each stable period) as fixed control variables, while male identity, primary male identity, and dyad identity were included as a random intercept in the model. The following random slope components were included: male-male association type and number of females associated with the unit within male identity and male-male association type within primary male identity.

Males associated with the primary male exchanged higher rates of ad libitum and focal interactions and spent higher proportions of time within $1 \mathrm{~m}$ of the primary male's females compared to males not associated with the primary male (see supplementary figure S4.2 and supplementary tables S4.16a, S4.16b, S4.16c).

\section{Definition of associated secondary males:}

Based on these results and following methodologies established for hamadryads baboons (Chowdhury et al., 2015; p. 503), we defined secondary males as non-primary adolescent/adult males who were most frequently within $1 \mathrm{~m}$ proximity of a primary male and interacted at significantly higher rates and spent a significantly higher proportion of time within $1 \mathrm{~m}$ of the primary male's females compared to other co-resident males. Note that secondary males could be associated with multiple units. 


\section{Appendix 4.2a Male dominance assessment - methodological details}

Based on Sánchez-Tójar et al. (2018), sampling effort was calculated as the ratio of interactions to individuals (i.e. number of interactions divided by number of individuals). Dataset sparseness was assessed comparing the proportion of known dyads (i.e. number of dyads that interacted / total number of possible dyads) and the expected proportion for a simulated population of equal group size where the probability of interacting followed a Poisson process. We used the "elo_scores" function to calculate the randomized Elo-scores (hereafter rElo) and obtain the mean ranks and respective $95 \%$ range of the rElo score values per individual (i.e. within $2.5 \%$ and $97.5 \%$ quantiles). We used the functions "plot_ranks" and "plot_hierarchy_shape" to plot the individual rElo scores and the hierarchy shapes, respectively. As described by Sánchez-Tójar et al. (2018), we quantified the steepness and uncertainty of the hierarchy from the rElo-rating repeatability using the function "estimate_uncertainty_by_repeatability", where higher repeatability scores correspond to the steepest hierarchies, and the "estimate_uncertainty_by_splitting" function, which splits the dataset in two and compares hierarchy estimates between the two subsets.

To assess the degree of hierarchy orderliness, we used the "ttri_test" function of the "compete" package (Curley, 2016; following the algorithm developed by Shizuka and Mcdonald, 2012;) to calculate the triangular transitivity (i.e. proportion of transitive closed triads out of the total number of closed triads; note that this calculation is not based on all possible dyads but only on all complete triads) and the respective $\mathrm{p}$ value for each party.

\section{Appendix 4.2b Male dominance assessment - detailed results}

Sampling effort was within the recommended range of values of 10 to 20 interactions per individual for both parties (but see below), with higher values for party 6 (ratio=18.9; $n=14 ; 264$ total interactions) than for party 9 (ratio $=14.1 ; n=10 ; 141$ total interactions). The number of dyads that never interacted during the study period was $8 \%$ for party 6 (89 total dyads) and 38\% for party 9 (45 total dyads) for a total of 134 dyads. For both parties the observed proportion of known dyads was within the expected range predicted by a Poisson process, specifically 0.73 for party 6 and 0.69 for party 9 with the respective expected average proportion of $0.57(2.5 \%-97.5 \%$ quantiles $=0.38-0.80)$ and 0.62 $(2.5 \%-97.5 \%$ quantiles $=0.42-0.89)$. This indicates that the data collection was not biased toward specific dyads for either party.

Mean randomized Elo-scores for each male of the two parties can be found in supplementary tables $\mathrm{S} 4.8 \mathrm{a}$ and $\mathrm{S} 4.8 \mathrm{~b}$, while the plot of the mean rElo scores and the respective $95 \%$ range of rElo scores can be found in supplementary figure S4.4. Differences between males of subsequent ranks were small and the $95 \%$ ranges of individual scores overlap to a large extent. Moreover, the large range of the $2.5 \%$ and $97.5 \%$ rElo score's quantiles indicates that rank varies greatly and/or is highly uncertain. 
The hierarchy shape plots (supplementary figure S4.5) indicate that neither party had a very steep hierarchy, with party 6 showing intermediate and party 9 low steepness. In party 6 rank predicted the winning probability for small rank differences only weakly (e.g. for differences in rank of 1-2 the average probability was only $\sim 0.55-0.65)$ while large rank differences were associated with a greater probability of winning (e.g. $\sim 0.80-1.00$, but see rank distance=8). In party 9 rank seemed to better predict winning probabilities for small rank differences (e.g. for differences in rank of 1 and 2 the average probability was $\sim 0.70$ and $\sim 0.90$ respectively) while larger rank differences seemed to be associated with a decrease in probability of winning (e.g. for differences in rank of 8 the average probability was $\sim 0.65$ ). Some of these patterns can be explained by the large size of the $2.5 \%$ and $97.5 \%$ quantile error bars in the plots, that indicate that both datasets could benefit from an increase in sampling effort. This is in accordance with the results of Sánchez-Tójar et al.'s simulation study (2018), which indicated that the general recommendations for a ratio of 10 to 20 interactions per individual generates meaningful hierarchy estimations in steep hierarchy scenarios, while more interactions might be required for intermediate and flat hierarchies. Specifically, for flat hierarchy scenarios the sampling effort should greatly exceed 100 to produce meaningful estimates.

The steepness/uncertainty assessment based on rElo-rating repeatability corroborates these findings with values of 0.817 for party 6 and 0.797 for party 9 . With higher repeatability scores $(\sim 1)$ corresponding to the steepest hierarchies, these values confirm that the hierarchies for parties 6 and 9 are of intermediate/low steepness. The hierarchy uncertainty assessment calculated by splitting the dataset in two subsets and comparing the respective rank estimates were 0.70 (2.5\%-97.5\% quantiles $=0.42-0.89)$ for party 6 and $0.66(2.5 \%-97.5 \%$ quantiles $=0.26-0.93)$ for party 9 . These values and the very large ranges of the quantiles show that our estimates are uncertain. In summary, the outcomes from these two methods show that both hierarchies are not steep and that our estimates are not very informative and not certain.

The triangular transitivity indices for both parties indicated that both hierarchies presented high transitivity (range from 0 to 1 where 1 indicates that all complete triads were transitive), with party 6 having an index of 0.82 and party 9 of 1.00 . For both parties this value was significantly higher than expected by chance with p-values of $<0.001$ and 0.001 for party 6 and 9 , respectively.

\section{Appendix 4.2c Male dominance assessment - discussion}

Overall, our results are in accordance with previous studies on this population (Patzelt et al., 2014; Kalbitzer et al., 2015; Fischer et al., 2017), which found that adult males present less consistent and clear dominance patterns compared to other Papio species (Kalbitzer et al., 2015). In our study we furthermore showed that male hierarchies were of intermediate/low steepness, individual rank scores were highly variable with great overlap between the 95\% score ranges, and, most importantly, estimates were uncertain. This uncertainty was mainly driven by the very low rate of aggression (Patzelt 
et al., 2014; Kalbitzer et al., 2015) and the high proportions of polyadic events and undecided interactions, that in turn resulted in a limited number of available interactions for the dominance assessment. Data from many years combined would need to be used in order to attain the adequate sampling power to obtain certain estimates in this species. However, clustering data collected over long time spans would not be meaningful as it would fail to take into account the important effect of demographical and social changes on dominance. These results and other past unsuccessful attempts to establish a significant linear hierarchy (Patzelt et al., 2014; Kalbitzer et al., 2015) indicate that steepness and linearity are not a key element of male dominance in this species. Moreover, the low levels of aggression seems to indicate that male competition and rank do not play a central role (Patzelt et al., 2014; Kalbitzer et al., 2015). This is unsurprising considering the social system of this species and the presence of stable male-female associations where the primary male monopolizes $98.6 \%$ of copulations (Goffe et al., 2016) and sires 89.5\% of offspring within their unit.

\section{Appendix 4.3 Genetic relatedness and paternity analyses}

\section{Samples, extraction and genotyping}

We collected fecal samples of all adolescent/adult males $(\mathrm{n}=24)$ and subadult/adult females $(\mathrm{n}=27)$ belonging to our two study parties for a total of 51 individuals. All offspring born during 2014 and 2015 were identified for a total of 24 infants. Of these, we were able to sample 19 offspring for paternity analyses, while 5 offspring deceased before we could sample them. Fecal samples were stored following a 2-step preservation protocol, with an initial phase of conservation in $90 \%$ ethanol followed by desiccation and storage with silica beads (Roeder et al., 2004). Samples were shipped to the German Primate Center in Germany every year and subsequently stored at $-20{ }^{\circ} \mathrm{C}$.

We extracted DNA from fecal samples using the First-DNA all tissue kit (Genial $\left.{ }^{\circledR}\right)$. Based on the methodologies used in Patzelt et al. (2014), we characterized genetic variation in Guinea baboons by assessing the individual allele variation on 24 polymorphic autosomal microsatellite markers. The 24 markers were amplified in 5 different multiplex systems (mean number of loci per multiplex, $4.80 \pm$ 1.10 SD) using the Multiplex PCR Kit (QIAGEN) and fluorescent-labelled primers in concentrations ranging from 0.05 to $0.5 \mu \mathrm{M}$. PCR cycling conditions included a hot start polymerase activation step at $95^{\circ} \mathrm{C}$ for $15 \mathrm{~min}$, followed by 40 cycles with denaturation at $94^{\circ} \mathrm{C}$ for 30 s, primer annealing at $57^{\circ} \mathrm{C}$ for 40 s, elongation at $72^{\circ} \mathrm{C}$ for 40 s, and a single final elongation step at $72^{\circ} \mathrm{C}$ for $30 \mathrm{~min}$. Detailed information regarding loci identities, primer sequences and concentrations, and fluorescent dyes are reported in supplementary table S4.5. PCR products were separated and detected through capillary gel electrophoresis on an ABI 3130xL Genetic Analyzer (16 capillary sequencer, Applied Biosystems ${ }^{\circledR}$, USA). Microsatellite allele sizes were evaluated in comparison with the GeneScanTM -400HD size standard using Gene Mapper 5 (Applied Biosystems ${ }^{\circledR}$ ). Allele calling for each locus was repeated until 5 completely consistent calls were achieved. To avoid contamination, every extraction, poly- 
merase chain reaction (PCR) and sequencing was performed in separate rooms and monitored with negative controls.

When tissue samples were available from previous studies (see Patzelt et al., 2014), at least one fecal sample was genotyped in order to cross-check individual identity. For all other individuals (except offspring) analyses were conducted on at least two independent fecal samples to rule out identification errors during sample collection (mean number of fecal samples: 3 per adolescent/adult male; 2.8 per subadult/adult female). As only one sample was collected for some offspring (mean number of fecal samples: 2.53), these samples were validated using a PCR-based sexing assay to confirm the reported sex (see supplementary appendix 4.4). All sexing assessments were in agreement with the known sex of the offspring.

We calculated descriptive statistics for all 24 markers in both genotype datasets for male-male relatedness and paternity analyses. We estimated FIS, expected and observed heterozygosity, and tested for Hardy-Weinberg equilibrium (HWE) for all loci using the R package PopGenReport (version 3.0.0; Gruber and Adamack, 2015; R version 3.4.4 and RStudio version 1.1.456). We tested for the presence of null alleles using MICRO-CHECKER (version 2.2.3; Van Oosterhout et al., 2004). All loci were polymorphic with allele numbers averaging 3.5 (range=2-6) and 3.8 (range=2-7) for the male-male relatedness and paternity dataset, respectively. One locus (D1s548) showed signs of null alleles in both datasets and significant deviations from HWE in the relatedness dataset (see supplementary tables S4.6a and S4.6b). Therefore, this locus was excluded and a total of 23 loci were included in the following relatedness and paternity analyses. All details regarding descriptive statistics of genetic markers can be found in the supplementary tables S4.6a and S4.6b.

\section{Relatedness estimation}

We estimated dyadic relatedness for all 24 adolescent and adult males belonging to parties 6 and 9 for a total of 134 within-party dyads. As relatedness estimators are influenced by the allele-frequency distributions and the true relationships of the individuals included in the dataset (Blouin, 2003; Pew et al., 2015), we used the R package "related" (Pew et al., 2015; also see Wang, 2011) to choose the best estimator. This package provides calculations and comparisons of five non-likelihood (Queller and Goodnight, 1989; Li et al., 1993; Ritland, 1996; Lynch and Ritland, 1999; Wang, 2002) and two likelihood estimators (Milligan, 2003; Wang, 2007). Based on the characteristics of the molecular markers of our dataset, we simulated 100 pairs of individuals each of known relatedness categories (parentoffspring, full-sibling, half-sibling, and unrelated). We then compared the observed and expected relatedness values for each of the seven relatedness estimators using Spearman's correlation. The Wang estimator (Wang, 2002) showed the best performance with the highest correlation coefficient, and was therefore selected as the best relatedness estimator in our male-male relatedness analysis. This estimator may range from -1 to 1 and should be interpreted as a correlation as originally conceived by 
Wright $(1921,1922)$, where negative estimates indicate pairs that are less related than average (Wang, 2017). Relatedness estimates for our male-male within party dyads averaged 0.063 (SD \pm 0.237 ) and ranged from -0.558 to 0.691 (median=0.048). Note that pedigree reconstruction analysis was not performed, as our genetic database is too recent to provide information on mother-offspring pairs for our adolescent and adult individuals.

\section{Paternity analysis}

We estimated paternity for all 19 sampled offspring born during 2014 and 2015 in our two parties (see table supplementary table S4.10) and assigned paternity using a likelihood approach with Cervus (version 3.0.7; Kalinowski et al., 2007). For all analyses we first ran a simulated parentage analysis with 10000 offspring followed by the actual parentage analysis. Confidence levels of parent assignments were set to $95 \%$ ("strict" criterion; while the "relaxed criterion" was $80 \%$ ). The following parameters were used in all calculations: proportion of potential sires sampled 0.95 , proportion of typed loci 1.00, and proportion of mistyped loci 0.01. All 27 females belonging to our two parties were included in this analysis. Transfer of mothers to another primary male's unit between time of conception and birth did not occur during the study period (see supplementary table S4.10). Identities of mothers were known from field observations, but we further checked all mother/offspring pairs with a maternity likelihood analysis. All known mothers were identified as candidates with 0 mismatches. Therefore, we ran a paternity analysis with a trio likelihood approach where the identity of the mother was known in order to determine the most likely father. All adolescent and adult males of our two parties were included as potential sires in this analysis. We included additional males from another known party of the same gang (party 4) because all potential sires were excluded for one offspring from party 9. One of these sires was identified as the most likely father with 0 mismatches. A male was considered to have sired an offspring when he was assigned as the most likely father, had 0 to maximum 1 mismatched alleles, and the confidence level for the assignments was more than $95 \%$, according to the "strict" criterion (see supplementary table S4.10).

\section{Appendix 4.4 Sex-determination protocol}

For sexing, two primers were used to amplify a region of Dead Box gene (F: GGA CGR ACT CTA GAT CGG, R: GTN CAG ATC TAR GAG GAA). The primers amplify one fragment in the female and two fragments in the male. Sexing-PCR was carried out in a $20 \mu \mathrm{l}$ volume using the QIAGEN

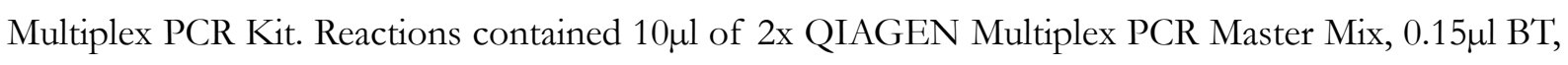
$1 \mu \mathrm{l}$ of each primer $(10 \mathrm{pM})$ and $0,5-5 \mu \mathrm{l}$ DNA. Water was added as needed to reach the final volume. PCR conditions comprised a pre-denaturing and polymerase activation step at $94^{\circ} \mathrm{C}$ for $15 \mathrm{~min}$, followed by $40-50$ cycles at $94^{\circ} \mathrm{C}$ for $30 \mathrm{sec}$, annealing at $58^{\circ} \mathrm{C}$ for $30 \mathrm{sec}$ and $72^{\circ} \mathrm{C}$ for $30 \mathrm{sec}$. A final extension step was carried out at $72^{\circ} \mathrm{C}$ for 5 minutes. Negative controls (only water added) and positive controls (high quality DNA of known male and female sex) were carried along for all amplifications. 
Sex determination was done by visual inspection of the PCR products on a 2.5\% Agarose gel stained with ethidium bromide.

\section{Appendix 4.5 Data analysis and modeling supplementary information}

All statistical analyses and visualizations were conducted using the $\mathrm{R}$ environment (version 3.4.4, $\mathrm{R}$ Development Core Team, 2018) in the RStudio interface (version 1.1.456, RStudio Team, 2018). We ran generalized linear mixed models using the R package "lme4" (Bates et al., 2015).

All covariates were z-transformed to a mean of zero and a standard deviation of one prior to fitting the models, to make the estimate comparison easier to interpret (Schielzeth, 2010). Information about original means and standard deviations can be found in the tables below. In the Gaussian models 4a, $4 \mathrm{~b}$ and 6 the response was transformed; details about the transformation type and the means and standard deviations of both original and transformed value can be found in the tables below. The use of non-default lmer/glmer optimizers is mentioned for each model when relevant (see details below).

In all relevant models the maximal random-effect structure including all possible random slope components was used to reduce type I error rates (Barr et al., 2013; see details for each model below). While we initially included correlations between random intercept and random slopes in all relevant models, these were either "unidentifiable" (i.e. absolute correlation parameter $\sim 1$ ) or the model did not converge. As model performance are similar between models with "fully-maximal" structure and models without random correlations and the latter were found to not severely compromise type I error rates (Barr et al., 2013), all our final models did not include random correlations.

Before performing inference all models were validated with several diagnostic checks. We assessed the assumption of normality for random effects components by visually inspecting each random intercept and slope histogram. The stability of each model was determined by comparing the estimates obtained from running models with the levels of the random effects excluded one at a time with the ones of the full model based on the full dataset (Quinn and Keough, 2002). Variance inflation factors for each predictor were calculated using the "vif" function of the package car (Fox and Weisberg, 2011; Field et al., 2012) on reduced general linear models with all random effect structures and optimizers excluded. The overall average VIF value for all our models was 1.363 with a standard deviation of 0.290 (range=1.001-1.726). As VIF values below 5 indicate no concern for multicollinearity (Bowerman and O'connell, 1990; Wooldridge, 2013), we ruled out collinearity for all our models. For all gaussian models the assumption of residual normality and homogeneity was assessed by visually inspecting histograms and qq-plots of residual distribution and scatterplots of residuals plotted against fitted values (Quinn and Keough, 2002). No obvious deviation from these assumptions was recorded. To check for potential type I errors due to overdispersion, we checked the dispersion parameter for all count models (Young et al., 1999; Crawley, 2002). All final full models were not overdispersed (see tables below for details). 
For all models with multiple predictors of interest (see details for each model below), we first determined the significance of the full model (also including all predictors of interest) against a null model comprising only the control predictors, the random factors (random intercept and random slope), and the intercept.

P-values were obtained from the likelihood ratio test performed with the R function "drop1" with argument "test" set to "chisq" (Barr et al., 2013). Effect sizes were calculated with the "summ" function of the "jtools" R package (version 1.1.1; Jacob and Long, 2018).

\section{Figure S4.1}

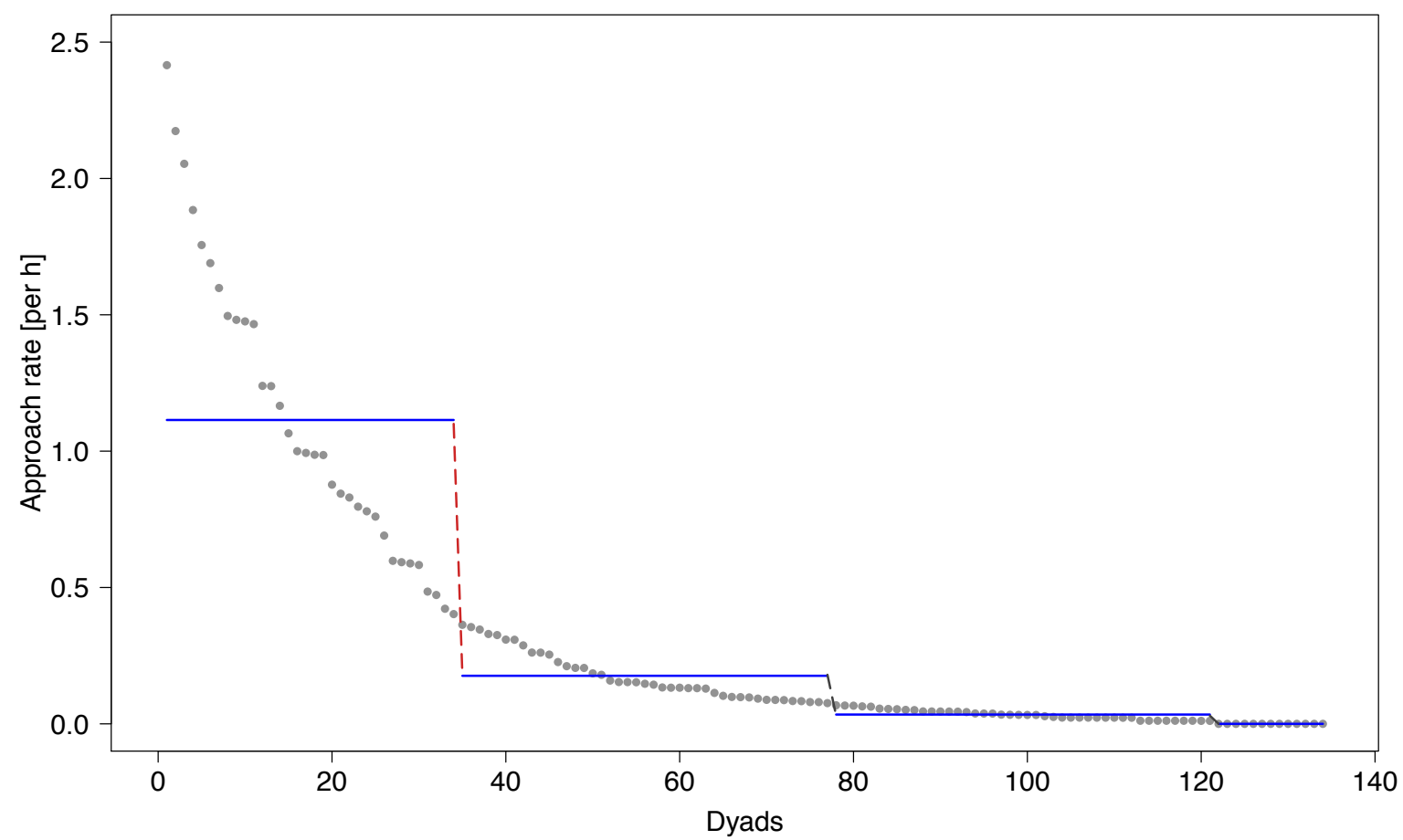

Plot illustrating the results of the changepoint analysis performed on the male-male approach rate per hour. Three main changepoint locations were identified (at dyad numbers 34, 77, and 121). We used the largest changepoint (first point at dyad number 34) as a threshold to identify associated and non-associated male-male dyads (see supplementary appendix 4.1). 


\section{Figure S4.2}
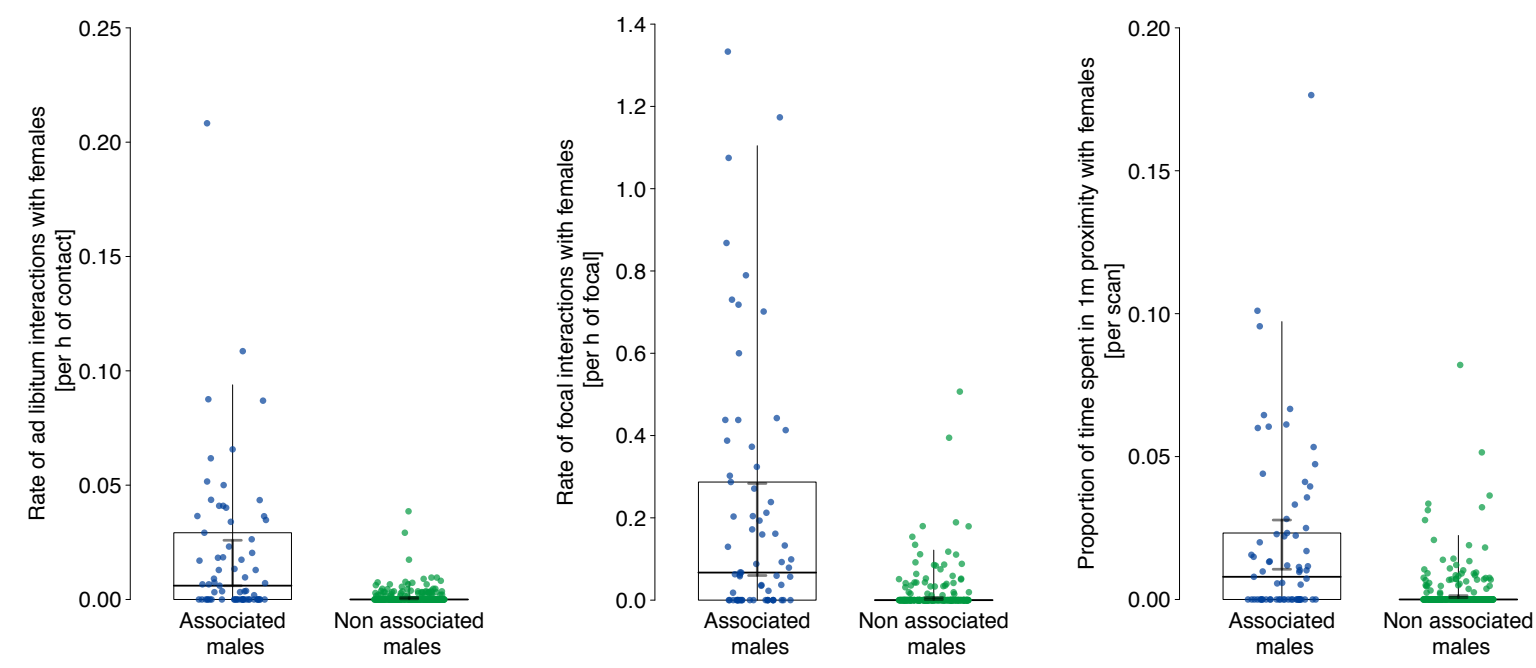

Per stable period: male rates of ad libitum interactions (a), focal interactions (b), and proportion of time spent in $1 \mathrm{~m}$ proximity (c) with the primary male's females by male-male association type (males associated vs. not associated with primary males). Associated males interacted at significantly higher rates and spent significantly higher proportions of time in close spatial association with the primary male's associated females (see supplementary appendix 4.1). Points depict each male for every stable period (total $n=24 ; 34$ stable periods). Boxplots depict the median, the lower and upper quartiles (25\% and 75\%), and the range excluding outliers (vertical line). Short horizontal whiskers within each box depict the bootstrapped $95 \%$ confidence intervals (number of females associated with the unit and party manually dummy coded and centered).

\section{Figure S4.3}

Year 2014

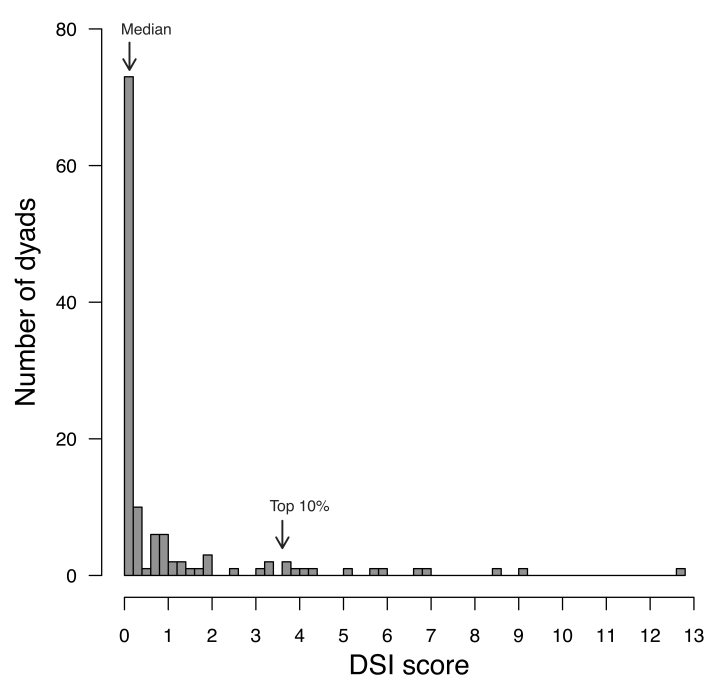

Year 2015

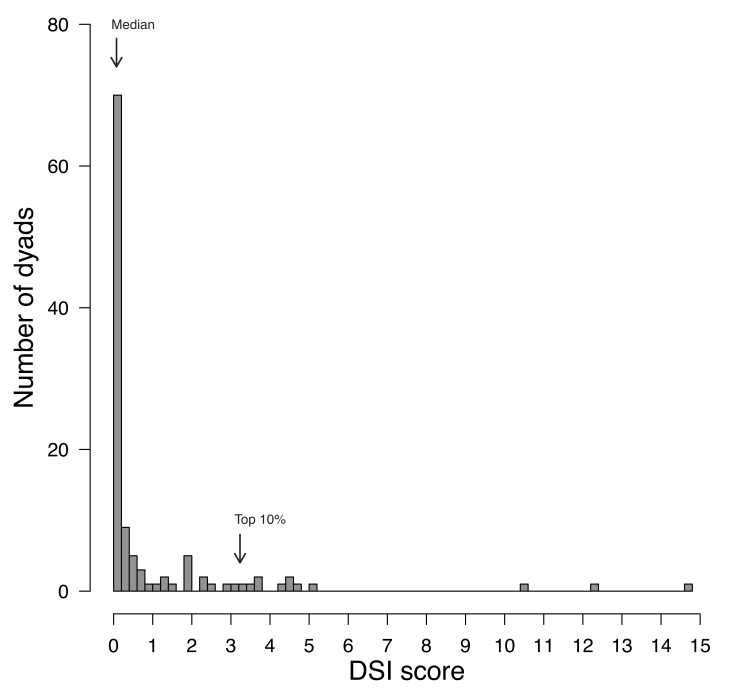

Distribution of the dyadic composite sociality index (DSI) calculated for year 2014 (left) and 2015 (right) for all male-male within-party dyads. This index measures the strength of dyadic male-male affiliative social relationships compared to the average of the group. Here DSI values from both study parties are pooled together (2014: mean $=1$, median $=0.115$, range $=0.000-12.785$; 2015: mean $=1$, median $=0.074$, range $=0.000-14.732$; see supplementary table $\mathrm{S} 4.7 \mathrm{a}$ for more details). 


\section{Figure S4.4}
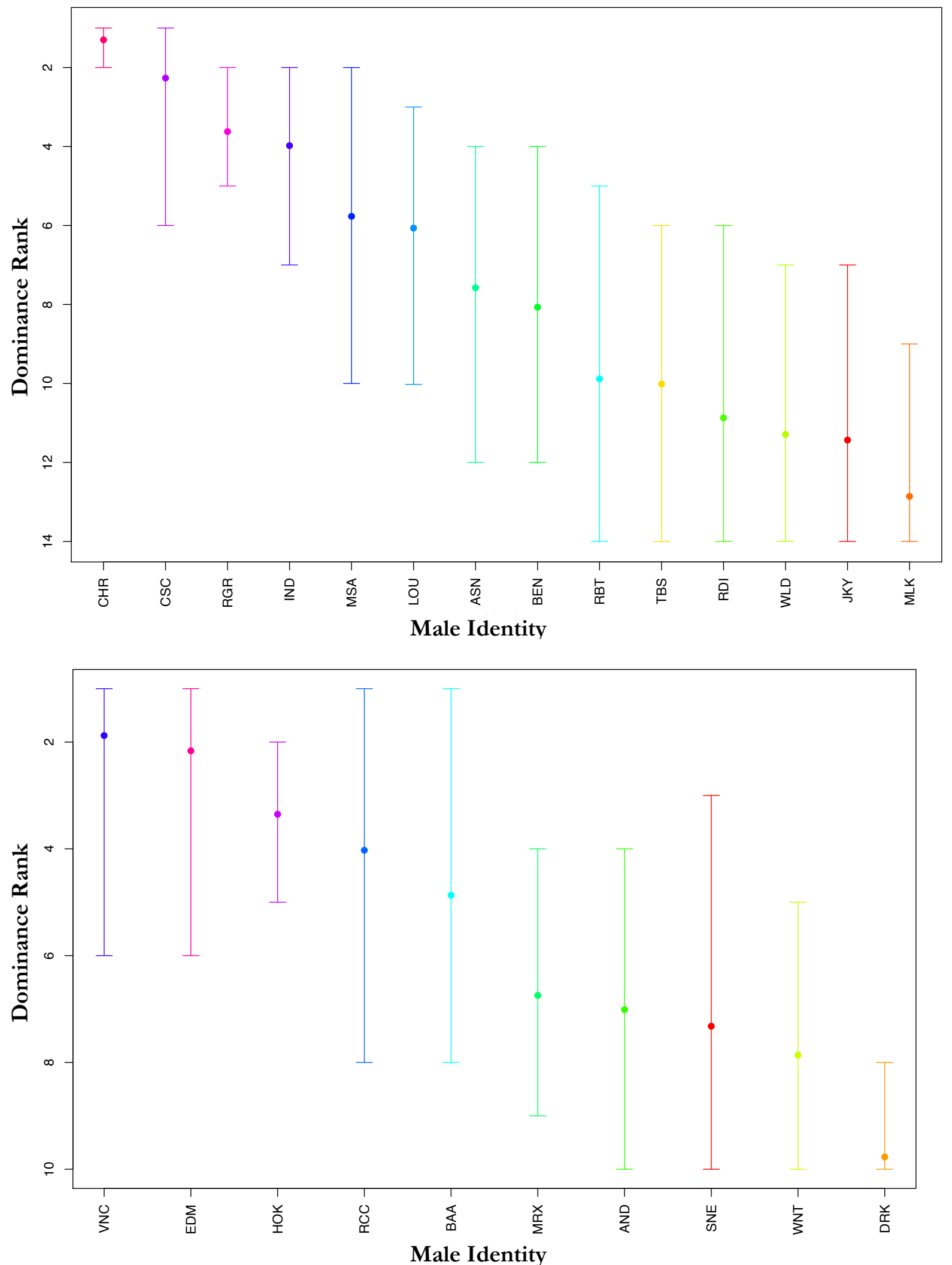

Randomized Elo-rating scores for male Guinea baboons of our two study parties (party 6, n=14, and party9, n=10, in the upper and lower plot respectively). Each point represents the average rElo-rating score per male with the respective $95 \%$ score range (i.e. within $2.5 \%$ and $97.5 \%$ quantiles). Note that the y axis representing dominance rank is reversed, with lower ranking individuals (i.e. rank=1) occurring at the top and higher ranking individuals (i.e. rank $=14$ and 10 respectively) at the bottom. Also see supplementary appendix $4.2 \mathrm{a}$ and $4.2 \mathrm{~b}$. 


\section{Figure S4.5}

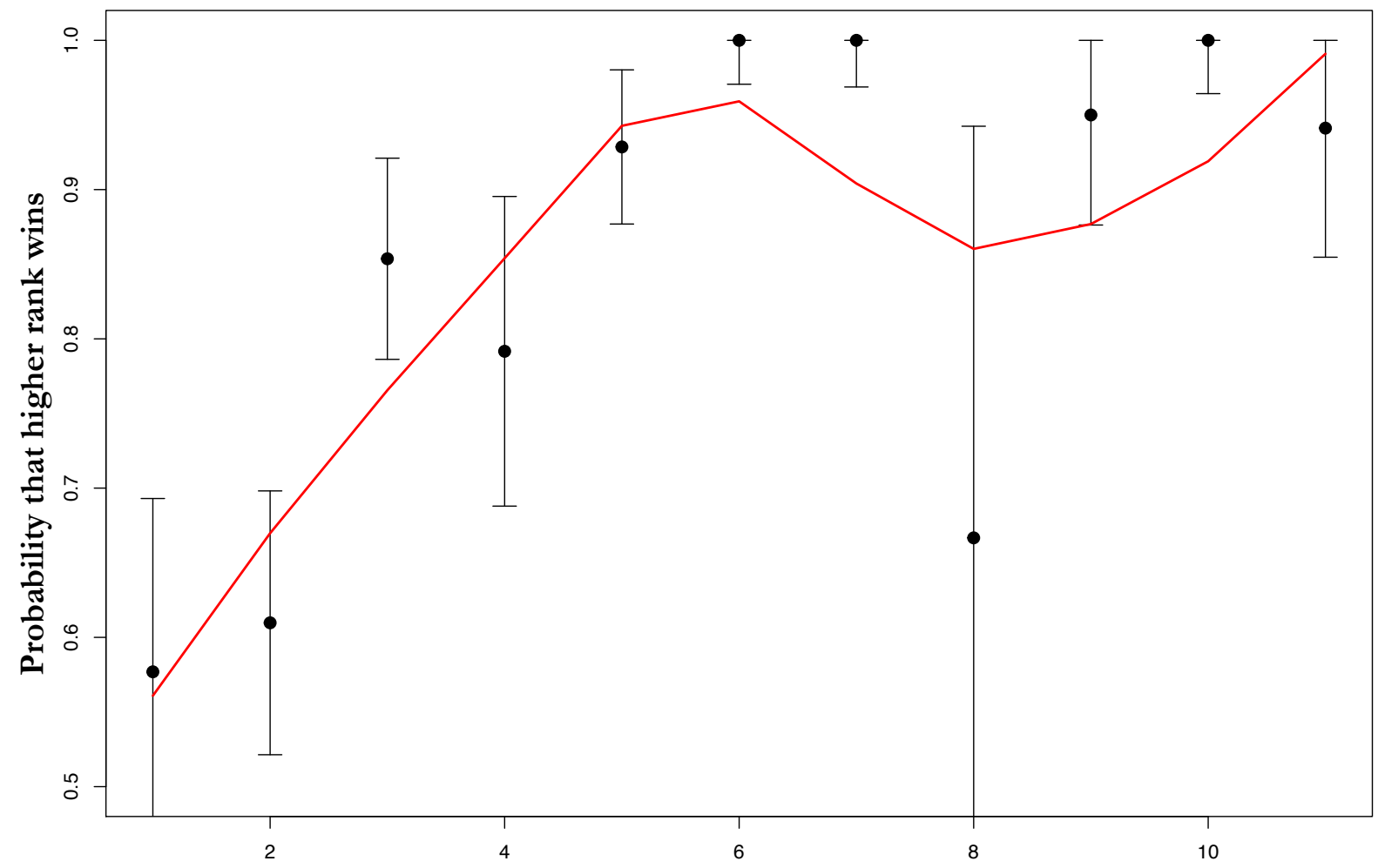

Difference in rank

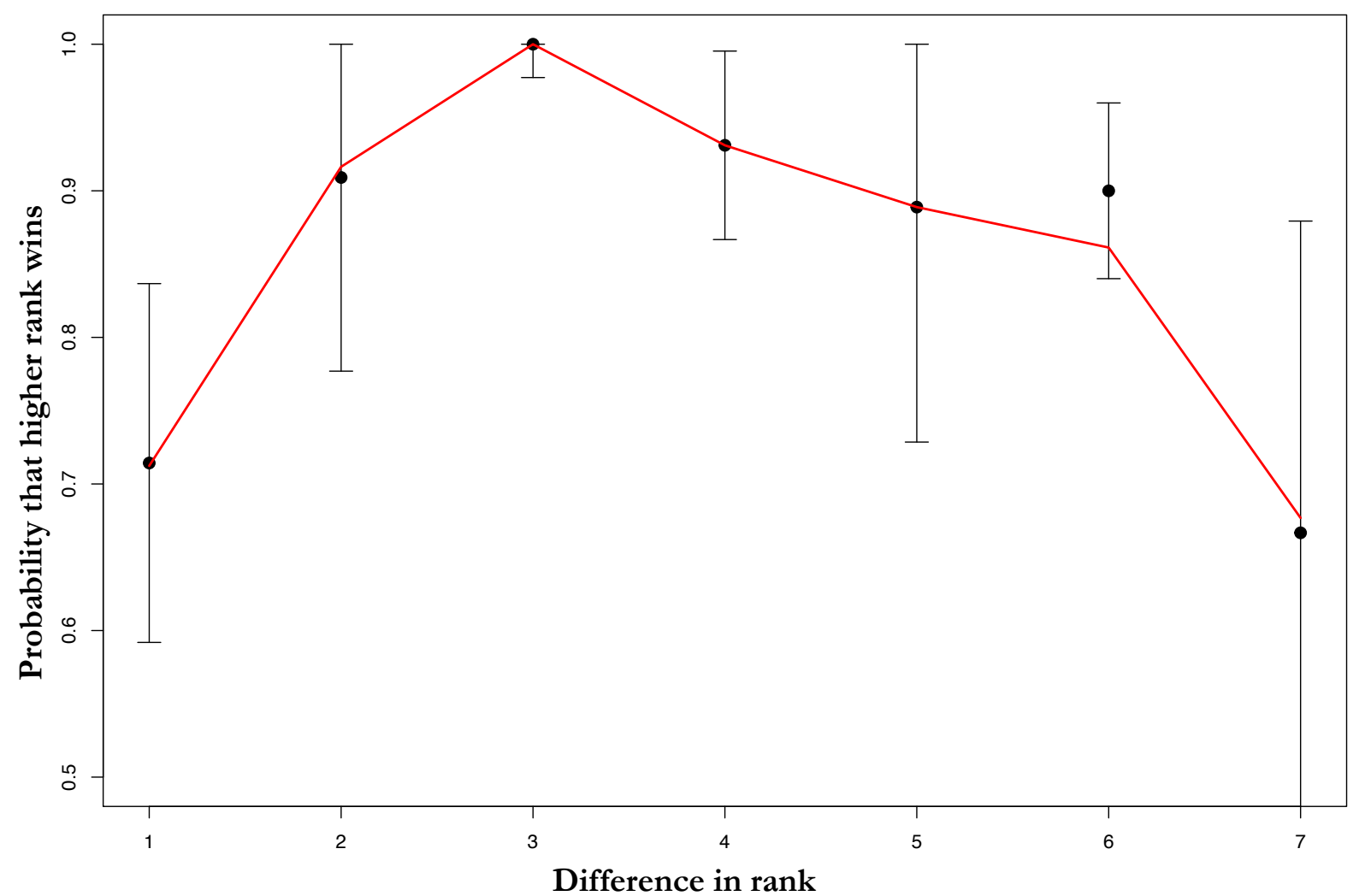

Hierarchy shape plots for male Guinea baboons of our two study parties (party $6, n=14$, and party9, $n=10$, in the upper and lower plot respectively). The plots illustrate the probability of higher ranking individuals winning dependent on the difference in rank between individuals. Also see supplementary appendix $4.2 \mathrm{a}$ and $4.2 \mathrm{~b}$. 


\section{Figure S4.6}

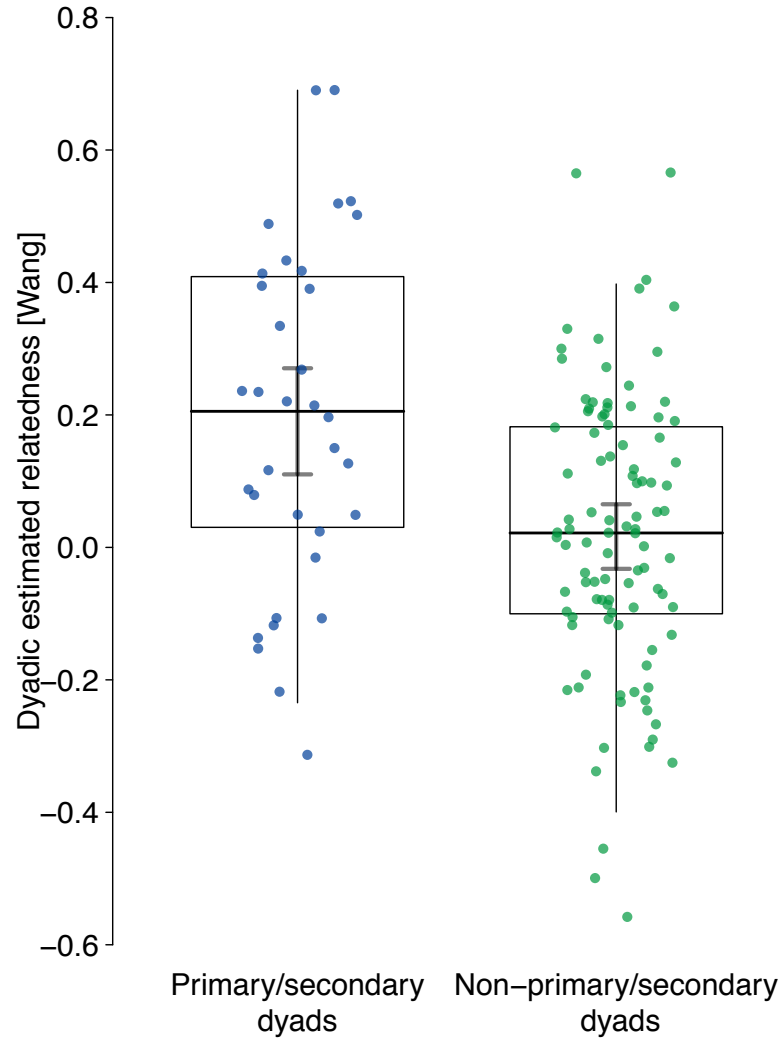

Average dyadic male-male relatedness estimates (wang) by relationship type 2 (Primary and their secondary males versus other male-male dyads, i.e. primary/secondary males versus Non-primary/secondary males). Dyads composed of primary males and their associated secondary males had significantly higher average relatedness estimates than all other male-male dyads. Points depict within-party male-male dyads (134 dyads, 24 males). Boxplots depict the median, the lower and upper quartiles $(25 \%$ and $75 \%)$, and the range excluding outliers (vertical line). Short horizontal whiskers within each box depict the bootstrapped 95\% confidence intervals (party manually dummy coded and centered).

Supplementary table S4.1a Average group composition of parties 6 and 9 during 2014 and 2015

\begin{tabular}{lll}
\hline Age/Sex Category & Party 6 & Party 9 \\
\hline Adult males & 8 (range: $7-9)$ & 5.5 (range: $5-6)$ \\
Adult females & 9 (range: $9-9)$ & 11.5 (range: $8-15)$ \\
Adolescent males & 4.5 (range: $4-5)$ & 3.5 (range: $3-4)$ \\
Subadult females & 0.5 (range: $0-1)$ & 2.5 (range: $1-4)$ \\
Middle/small juveniles & 16 (range: $14-18)$ & 15 (range: $13-17)$ \\
Yearlings/infants & 6.5 (range: 5-8) & 6.5 (range: $4-9)$ \\
\hline
\end{tabular}


Supplementary table S4.1b Average group composition of parties 6 and 9 during 2016 and 2017 (this period was only used for the relationship stability analysis)

\begin{tabular}{lll}
\hline Age/Sex Category & Party 6 & Party 9 \\
\hline Adult males & 10 (range: $10-10)$ & 7 (range: $7-7)$ \\
Adult females & 8.5 (range: $8-9)$ & 14.5 (range: $14-15)$ \\
Adolescent males & 2 (range: $2-2)$ & 3.5 (range: $3-4)$ \\
Subadult females & 1.5 (range: $1-2)$ & 1 (range: $1-1)$ \\
Middle/small juveniles & 13.5 (range: $7-20)$ & 11.5 (range: $11-12)$ \\
Yearlings/infants & 5.5 (range: $5-6)$ & 10 (range: $9-11)$ \\
\hline
\end{tabular}

\section{Supplementary table S4.2a Table of sex/age category definitions}

\begin{tabular}{|c|c|c|}
\hline \multirow{3}{*}{ 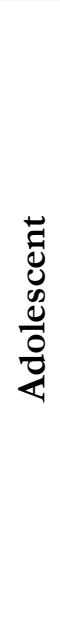 } & "Large juvenile male" & $\begin{array}{l}\text { When juvenile males reach a bigger size than mature adult } \\
\text { females but smaller than adult males (closer in size to adult } \\
\text { females). They may experience testicular enlargement. Canines } \\
\text { extending beyond tooth row and mantle starting to develop } \\
\text { marks the end of this period. }\end{array}$ \\
\hline & "Small subadult male" & $\begin{array}{l}\text { Not yet attained full body size. Secondary sexual characteristics } \\
\text { are partially but not fully developed (mantle, canine ridges, long } \\
\text { canine teeth). The scrotum is enlarged. They are visibly bigger } \\
\text { than adult females and large juvenile males, but still have a lanky } \\
\text { appearance and are smaller than adult males. }\end{array}$ \\
\hline & "Large subadult male" & $\begin{array}{l}\text { Begins when individual no longer has a lanky appearance but } \\
\text { has not yet attained full body size or muscle mass. Body shape is } \\
\text { more similar to the one of adult than large juvenile or small } \\
\text { subadult males. }\end{array}$ \\
\hline \multirow{4}{*}{$\frac{ \pm}{\frac{7}{2}}$} & "Early-prime adult male" & $\begin{array}{l}\text { Secondary sexual characteristics and body size fully developed. } \\
\text { The coat is long and shiny. The ischial callosities become square } \\
\text { and wide and the butt may take on reddish color. Teeth in } \\
\text { category } 5 \text {. }\end{array}$ \\
\hline & "Mid-prime adult male" & $\begin{array}{l}\text { The mantle may show some breaks. The teeth start decaying } \\
\text { (categories } 3 \text { or } 4 \text { ). }\end{array}$ \\
\hline & "Late-prime adult male" & $\begin{array}{l}\text { The mantle starts to thin out. The male has less muscle mass. } \\
\text { Teeth in categories } 2 \text { or } 3 \text {. }\end{array}$ \\
\hline & "Old adult male" & $\begin{array}{l}\text { The mantle thins out visibly. The male has lost most of his } \\
\text { muscle mass. Teeth in categories } 1 \text { or } 2 \text {. }\end{array}$ \\
\hline
\end{tabular}

Supplementary table S4.2b Table of tooth status category definitions (from Kitchen et al., 2003)

\begin{tabular}{cl}
\hline Tooth Category & Definition \\
\hline 5 & $\begin{array}{l}\text { White teeth with sharp unchipped points. } \\
\text { White teeth or slight yellowing on one or two teeth, some chipping or } \\
\text { wear on one tooth. }\end{array}$ \\
3 & $\begin{array}{l}\text { Some discoloration on several teeth, breaks chipping or tooth wear very } \\
\text { evident. }\end{array}$ \\
2 & $\begin{array}{l}\text { Extensive discoloration, one or both canines missing or broken. } \\
\text { Extensive discoloration, one or both canines missing or worn to the level } \\
\text { of premolars and substantial damage to other teeth. }\end{array}$ \\
\hline
\end{tabular}


Supplementary table S4.3 Yearly data collection details from 2014 to 2017

\begin{tabular}{lcccc}
\hline Category & $\mathbf{2 0 1 4}$ & $\mathbf{2 0 1 5}$ & $\mathbf{2 0 1 6}$ & $\mathbf{2 0 1 7}$ \\
\hline Number of months & 9 & 10 & 6 & 9 \\
$\begin{array}{l}\text { Focal protocols } \\
\text { duration in hours) }\end{array}$ & 318 & 638 & 175 & 243 \\
Focal protocols (number) & 994 & 1967 & 539 & 744 \\
Proximity scans (number) & 1970 & 3941 & 1181 & 1401 \\
\hline
\end{tabular}

Supplementary table S4.4a Descriptive statistics for all behavioral components included in the DSI calculation for each time period. Average, standard deviation, minimum and maximum are reported for each behavioral component (mean $\pm \mathrm{SD}$ (min-max)). Dyadic behavioral rates per hour were calculated by dividing the count of dyadic interactions by the dyadic focal time in hours, while dyadic proportions of time spent performing a certain behavior were calculated by dividing the dyadic durations of the behavior by the dyadic focal time.

\begin{tabular}{lccc}
\hline Behavioral component & 2014 & 2015 & 2014 and 2015 \\
\hline Grooming rate (per h) & $0.047 \pm 0.123$ & $0.043 \pm 0.145$ & $0.045 \pm 0.130$ \\
& $(0.000-0.787)$ & $(0.000-1.338)$ & $(0.000-0.993)$ \\
Grooming proportion of time & $0.002 \pm 0.005$ & $0.001 \pm 0.004$ & $0.002 \pm 0.005$ \\
& $(0.000-0.042)$ & $(0.000-0.036)$ & $(0.000-0.042)$ \\
Contact-sit rate (per h) & $0.035 \pm 0.075$ & $0.061 \pm 0.128$ & $0.055 \pm 0.116$ \\
& $(0.000-0.409)$ & $(0.000-0.847)$ & $(0.000-0.594)$ \\
Contact-sit proportion of time & $0.001 \pm 0.002$ & $0.001 \pm 0.003$ & $0.001 \pm 0.002$ \\
& $(0.000-0.015)$ & $(0.000-0.020)$ & $(0.000-0.014)$ \\
Approach (within 1m) rate (per h) & $0.160 \pm 0.275$ & $0.383 \pm 0.571$ & $0.318 \pm 0.482$ \\
& $(0.000-1.465)$ & $(0.000-2.619)$ & $(0.000-2.263)$ \\
\hline
\end{tabular}

Supplementary table S4.4b Results from the Kendall's tau correlation test between all behavioral components included in the DSI calculation. Average, standard deviation, minimum, maximum, and relative p-value of the Tau coefficients calculated from correlations of all possible component combinations per period.

\begin{tabular}{lllll}
\hline Kendall's tau correlation test & $\mathbf{2 0 1 4}$ & $\mathbf{2 0 1 5}$ & 2014 and 2015 \\
\hline \multirow{4}{*}{ Tau coefficient } & Average & 0.62 & 0.69 & 0.69 \\
& Standard deviation & 0.18 & 0.15 & 0.14 \\
& Minimum & 0.51 & 0.50 & 0.56 \\
& Maximum & 0.97 & 0.98 & 0.97 \\
& P-value & All $<0.001$ & All $<0.001$ & All $<0.001$
\end{tabular}


Supplementary table S4.5 List of 24 microsatellite loci used in this study: multiplex-PCR, loci identities, primer sequences, fluorescence dye, and primer concentration

\begin{tabular}{|c|c|c|c|c|}
\hline $\begin{array}{l}\text { Multiple } \\
\text { x PCR }\end{array}$ & Locus ID & Primer 5'-3' & Fluoresc. & $\begin{array}{l}\text { Primer } \\
\text { Conc. (uM) }\end{array}$ \\
\hline M1 & D6S264F & AGC TGA CTT TAC GCT GTT C & Fam & 0.05 \\
\hline \multirow[t]{7}{*}{$57^{\circ} \mathrm{C}$} & D6S264R & TTT TCC ATG CCC TTC TAT CA & Fam & 0.05 \\
\hline & $\mathrm{D} 7 \mathrm{~S} 503 \mathrm{~F}$ & ATG ACT TGG AGT AAT GGG AG & Tamra & 0.15 \\
\hline & D7S503R & GTC CCT GAA AAC CTT TAA TCA & Tamra & 0.15 \\
\hline & $\mathrm{D} 12 \mathrm{~S} 375 \mathrm{~F}$ & TTG TTG AGG GTC TTT CTC CA & Fam & 0.09 \\
\hline & $\mathrm{D} 12 \mathrm{~S} 375 \mathrm{R}$ & ТСТ ТСТ TAT TTG GAA AAG TAA C & Fam & 0.09 \\
\hline & D3S1766F & ACC ACA TGA GCC AAT TCT GT & Tamra & 0.05 \\
\hline & D3S1766R & ACC CAA TTA TGG TGT TGT TAC & Tamra & 0.05 \\
\hline M2 & D14S306F & TCA GCT ACA TCC AAA TTA GGT & Tamra & 0.05 \\
\hline \multirow[t]{7}{*}{$57^{\circ} \mathrm{C}$} & D14S306R & TGA CAA AGA AAC TAA AAT GTC C & Tamra & 0.05 \\
\hline & D1S533F & TAT CСС ССС САA АAA ТАТ АТА & Fam & 0.05 \\
\hline & D1S533R & TTG CTA ACC AAA ATA ACA ATG GG & Fam & 0.05 \\
\hline & D2S1329F & TTG TAG AAC CСТ CTC AAA TAT & Tamra & 0.5 \\
\hline & D2S1329R & GAA ACT TCC ACC CTG GGT T & Tamra & 0.5 \\
\hline & D2S1326F & AGA CAG TCA AGA ATA ACT GC & Hex & 0.05 \\
\hline & D2S1326R & CTG TGA CCC AAA AGC CGA & Hex & 0.05 \\
\hline M3 & D10S611F & TAT ACA GGA AAC TGT GTA GTG & Tamra & 0.2 \\
\hline \multirow[t]{11}{*}{$57^{\circ} \mathrm{C}$} & D10S611R & CTA TAT TTA TGT GTG TGG ATG & Tamra & 0.2 \\
\hline & D8S1106F & TTG TTT ACC CСT GCA CCA C & Hex & 0.2 \\
\hline & D8S1106R & TTC TCA GAA TTG CTC ATA GTG & Hex & 0.2 \\
\hline & D17S791F & ATG TTC TCC AGT TAT TCC CC & Tamra & 0.5 \\
\hline & D17S791R & GCT GGT CCT TTG GAA GAG T & Tamra & 0.5 \\
\hline & D6S501F & GCT GGA AAC TGA TAA GGG C & Hex & 0.2 \\
\hline & D6S501R & GCC ACC CTG GCT AAG TTA & Hex & 0.2 \\
\hline & D17S1290F & GAC AAC AGA GCA AGA CTG T & Fam & 0.25 \\
\hline & D17S1290R & AGA AGC AGT TAA ATG GCC AAA & Fam & 0.25 \\
\hline & D6S311F & ATG TCC TCA TTT GTG TTG TG & Tamra & 0.3 \\
\hline & D6S311R & GAT TCA GAG CCC AGG AAG A & Tamra & 0.3 \\
\hline M4 & D5S1457F & TAG GTT CTT GGC ATG TCT GT & Tamra & 0.2 \\
\hline \multirow[t]{3}{*}{$57^{\circ} \mathrm{C}$} & D5S1457R & TGC TTG GCA TAC TTC AGG G & Tamra & 0.2 \\
\hline & D8S505F & CTA AAG TGA ACC CAA ACC TAA & Fam & 0.15 \\
\hline & D8S505R & AGT GCT AAG TCC CAG ACC A & Fam & 0.15 \\
\hline
\end{tabular}




\begin{tabular}{|c|c|c|c|c|}
\hline & D10S1432F & CAG TGG ACA CCA AAC ACA AT & Tamra & 0.4 \\
\hline & D10S1432R & TAG GTT ATC TAA ATA GTG GAT TT & Tamra & 0.4 \\
\hline & D5S820F & ATT GCA TGG CAA CTC TTC TC & Fam & 0.3 \\
\hline & D5S820R & GTT CTT CAG AGA AAC AGA AC & Fam & 0.3 \\
\hline & D3S1768F & GGT TGC TGC CAA AGA TTA GA & Hex & 0.15 \\
\hline & D3S1768R & CAC TGT AAT TTG CTG TTG GAT & Hex & 0.15 \\
\hline & D7S2204F & TCA TGA CAA AAC AGA AAA TAA GT & Fam & 0.4 \\
\hline & D7S2204R & AGT AAA TGG AAT TGC TTG TTA C & Fam & 0.4 \\
\hline M5 & D1S207F & CAC TTC TCC TTG AAT CGC T'T & Hex & 0.1 \\
\hline \multirow[t]{7}{*}{$57^{\circ} \mathrm{C}$} & D1S207R & GCA AGT CCT GTT CCA AGT C & Hex & 0.1 \\
\hline & D4S243F & TCA GTC TCT CTT TCT CCT TG & Fam & 0.15 \\
\hline & D4S243R & TAG GAG CCT GAG GTC CTG & Fam & 0.15 \\
\hline & D1S548F & GAA CTC AT'T GGC AAA AGG AA & Hex & 0.15 \\
\hline & D1S548R & GCC TCT TTG TTG CAG TGA TT & Hex & 0.15 \\
\hline & D21S1442F & СТС СТС ССС АСТ GCA GAT & Fam & 0.5 \\
\hline & D21S1442R & TCT CCA GAA TCA CAT GAG C & Fam & 0.5 \\
\hline
\end{tabular}

Supplementary table S4.6a Characteristics of the 24 microsatellite loci used to estimate male-male dyadic relatedness (calculated using the genotypes of all males of parties 6 and 9; $\mathrm{n}=24)$

\begin{tabular}{cccccccccccc}
\hline \multicolumn{2}{c}{ Loci } & \multicolumn{2}{c}{ Alleles } & $\begin{array}{c}\text { Inbree- } \\
\text { ding }\end{array}$ & \multicolumn{2}{c}{ Heterozygosity } & NAFE & $\begin{array}{c}\text { Null } \\
\text { alleles }\end{array}$ \\
\hline $\begin{array}{c}\text { Locus } \\
\text { ID }\end{array}$ & $\begin{array}{c}\text { Locus } \\
\text { No. }\end{array}$ & $\begin{array}{c}\text { Allele } \\
\text { range }\end{array}$ & $\begin{array}{c}\text { No. } \\
\text { All- } \\
\text { eles }\end{array}$ & FIs & He & Ho & $\begin{array}{c}\text { HWE } \\
*\end{array}$ & $\begin{array}{c}\text { Brook } \\
\text {-field }\end{array}$ & $\begin{array}{c}\text { Chakra- } \\
\text { borty }\end{array}$ & $\begin{array}{c}\text { Pres- } \\
\text { ence }\end{array}$ \\
\hline D6s264 & Locus1 & $94-98$ & 3 & 0.045 & 0.510 & 0.500 & 0.648 & 0.006 & 0.010 & no \\
\hline D7s503 & Locus2 & $152-166$ & 5 & 0.043 & 0.765 & 0.708 & 0.501 & 0.032 & 0.038 & no \\
\hline D12s375 & Locus3 & $165-181$ & 5 & -0.148 & 0.702 & 0.792 & 0.629 & -0.053 & -0.060 & no \\
\hline D3s1766 & Locus4 & $195-203$ & 2 & -0.092 & 0.187 & 0.208 & 1.000 & -0.018 & -0.055 & no \\
\hline D14s306 & Locus5 & $169-177$ & 3 & 0.060 & 0.520 & 0.500 & 1.000 & 0.013 & 0.020 & no \\
\hline D1s533 & Locus6 & $191-199$ & 3 & 0.071 & 0.602 & 0.583 & 0.432 & 0.011 & 0.015 & no \\
\hline D2s1329 & Locus7 & $212-224$ & 4 & -0.006 & 0.573 & 0.583 & 0.039 & -0.007 & -0.009 & no \\
\hline D2s1326 & Locus8 & $251-255$ & 2 & 0.005 & 0.413 & 0.417 & 1.000 & -0.003 & -0.004 & no \\
\hline D10s611 & Locus9 & $133-141$ & 3 & -0.085 & 0.555 & 0.583 & 0.057 & -0.018 & -0.025 & no \\
\hline D8s1106 & Locus10 & $148-156$ & 3 & -0.178 & 0.344 & 0.417 & 1.000 & -0.054 & -0.096 & no
\end{tabular}




\begin{tabular}{|c|c|c|c|c|c|c|c|c|c|c|}
\hline D17s791 & Locus11 & $166-170$ & 3 & 0.003 & 0.617 & 0.625 & 0.093 & -0.005 & -0.006 & no \\
\hline D6s501 & Locus12 & $171-188$ & 5 & -0.190 & 0.653 & 0.792 & 0.212 & -0.084 & -0.096 & no \\
\hline D17s1290 & Locus13 & $195-203$ & 3 & -0.001 & 0.369 & 0.375 & 1.000 & -0.004 & -0.008 & no \\
\hline D6s311 & Locus14 & $228-230$ & 2 & -0.166 & 0.430 & 0.458 & 1.000 & -0.020 & -0.032 & no \\
\hline D5s1457 & Locus15 & $128-132$ & 2 & -0.269 & 0.305 & 0.375 & 0.557 & -0.054 & -0.103 & no \\
\hline D8s505 & Locus16 & $147-151$ & 2 & -0.095 & 0.153 & 0.167 & 1.000 & -0.012 & -0.044 & no \\
\hline D10s1432 & Locus17 & $158-170$ & 6 & 0.022 & 0.768 & 0.708 & 0.199 & 0.034 & 0.041 & no \\
\hline D5s820 & Locus18 & 179-199 & 6 & 0.108 & 0.771 & 0.708 & 0.903 & 0.035 & 0.042 & no \\
\hline D3s1768 & Locus19 & $197-209$ & 3 & -0.004 & 0.617 & 0.625 & 0.724 & -0.005 & -0.006 & no \\
\hline $\mathrm{D} 7 \mathrm{~s} 2204$ & Locus 20 & $232-244$ & 4 & 0.017 & 0.689 & 0.667 & 0.029 & 0.013 & 0.017 & no \\
\hline D1s207 & Locus 21 & $133-135$ & 2 & -0.441 & 0.457 & 0.625 & 0.184 & -0.115 & -0.155 & no \\
\hline $\mathrm{D} 4 \mathrm{~s} 243$ & Locus22 & $151-163$ & 4 & -0.201 & 0.593 & 0.708 & 0.084 & -0.073 & -0.089 & no \\
\hline D1s548 & Locus 23 & 192-208 & 5 & 0.167 & 0.667 & 0.458 & 0.001 & 0.125 & 0.185 & yes \\
\hline D21s1142 & Locus24 & $230-242$ & 4 & -0.199 & 0.655 & 0.667 & 0.281 & -0.007 & -0.009 & no \\
\hline \multicolumn{2}{|c|}{ Mean } & - & 3.5 & -0.064 & 0.538 & 0.552 & - & -0.011 & -0.018 & - \\
\hline \multicolumn{2}{|c|}{$\mathrm{SD}$} & - & 1.3 & 0.139 & 0.175 & 0.168 & - & 0.048 & 0.067 & - \\
\hline \multicolumn{2}{|c|}{ Min } & - & 2.0 & -0.441 & 0.153 & 0.167 & - & -0.115 & -0.155 & - \\
\hline \multicolumn{2}{|c|}{ Max } & - & 6.0 & 0.167 & 0.771 & 0.792 & - & 0.125 & 0.185 & - \\
\hline
\end{tabular}

$\mathrm{F}_{\mathrm{IS}}=$ inbreeding coefficient according to Nei (1987); He= expected heterozygosity; Ho= observed heterozygosity; HWE $=$ Hardy-Weinberg equilibrium ( $*$ note that p-value was corrected for multiple testing with the Bonferroni adjustment, $\alpha=(0.05 / 24)=0.00208)$; NAFE $=$ null alleles frequencies estimators calculated based on Brookfield (1996) and Chakraborty et al. (1992) and presence of null alleles. (see supplementary appendix 4.3 for details about these calculations).

Supplementary table S4.6b Characteristics of the 24 microsatellite loci used to estimate paternity for 19 offspring (calculated using the genotypes of all males, females and offspring included in the analysis; $n=74$ )

\begin{tabular}{ccccccccccccc}
\hline \multicolumn{2}{c}{ Loci } & \multicolumn{2}{c}{ Alleles } & $\begin{array}{c}\text { Inbree- } \\
\text { ding }\end{array}$ & \multicolumn{2}{c}{ Heterozygosity } & \multicolumn{2}{c}{ NAFE } & $\begin{array}{c}\text { Null } \\
\text { alleles }\end{array}$ \\
\hline $\begin{array}{c}\text { Locus } \\
\text { ID }\end{array}$ & $\begin{array}{c}\text { Locus } \\
\text { No. }\end{array}$ & $\begin{array}{c}\text { Allele } \\
\text { range }\end{array}$ & $\begin{array}{c}\text { No. } \\
\text { All- } \\
\text { eles }\end{array}$ & FIs & He & Ho & $\begin{array}{c}\text { HWE } \\
*\end{array}$ & $\begin{array}{c}\text { Brook- } \\
\text { field }\end{array}$ & $\begin{array}{c}\text { Chakra- } \\
\text { borty }\end{array}$ & $\begin{array}{c}\text { Pres- } \\
\text { ence }\end{array}$ \\
\hline D6s264 & Locus1 & $94-98$ & 3 & 0.012 & 0.524 & 0.521 & 0.799 & 0.005 & 0.008 & no \\
D7s503 & Locus2 & $152-166$ & 5 & -0.033 & 0.755 & 0.732 & 0.458 & 0.009 & 0.011 & no \\
D12s375 & Locus3 & $165-181$ & 5 & -0.058 & 0.715 & 0.761 & 0.902 & -0.031 & -0.035 & no \\
D3s1766 & Locus4 & $195-203$ & 2 & -0.076 & 0.262 & 0.282 & 1.000 & -0.017 & -0.039 & no \\
\hline
\end{tabular}




\begin{tabular}{|c|c|c|c|c|c|c|c|c|c|c|}
\hline $\mathrm{D} 14 \mathrm{~s} 306$ & Locus5 & 169-177 & 3 & 0.005 & 0.533 & 0.535 & 0.896 & -0.006 & -0.009 & no \\
\hline D1s533 & Locus6 & 191-199 & 3 & 0.063 & 0.563 & 0.535 & 0.744 & 0.013 & 0.019 & no \\
\hline $\mathrm{D} 2 \mathrm{~s} 1329$ & Locus7 & $212-224$ & 4 & 0.030 & 0.560 & 0.549 & 0.125 & -0.003 & -0.004 & no \\
\hline D2s1326 & Locus8 & $251-255$ & 3 & -0.036 & 0.396 & 0.408 & 0.348 & -0.010 & -0.017 & no \\
\hline D10s611 & Locus9 & 133-141 & 3 & -0.189 & 0.561 & 0.620 & 0.008 & -0.038 & -0.050 & no \\
\hline D8s1106 & Locus10 & $148-156$ & 3 & -0.093 & 0.411 & 0.437 & 1.000 & -0.023 & -0.038 & no \\
\hline D17s791 & Locus11 & $166-170$ & 3 & -0.049 & 0.586 & 0.606 & 0.061 & -0.007 & -0.009 & no \\
\hline D6s501 & Locus12 & $171-188$ & 5 & -0.182 & 0.655 & 0.775 & 0.025 & -0.067 & -0.078 & no \\
\hline D17s1290 & Locus13 & $195-203$ & 4 & -0.086 & 0.428 & 0.465 & 0.939 & -0.013 & -0.020 & no \\
\hline D6s311 & Locus14 & $228-230$ & 2 & -0.185 & 0.371 & 0.408 & 0.554 & -0.017 & -0.030 & no \\
\hline D5s1457 & Locus15 & $128-132$ & 3 & -0.231 & 0.395 & 0.479 & 0.107 & -0.063 & -0.100 & no \\
\hline D8s505 & Locus16 & $147-151$ & 2 & -0.110 & 0.290 & 0.324 & 0.654 & -0.031 & -0.062 & no \\
\hline D10s1432 & Locus17 & $158-170$ & 7 & -0.041 & 0.764 & 0.746 & 0.585 & 0.004 & 0.004 & no \\
\hline D5s820 & Locus18 & 179-199 & 6 & 0.023 & 0.770 & 0.746 & 0.779 & 0.007 & 0.008 & no \\
\hline D3s1768 & Locus19 & $197-209$ & 4 & 0.098 & 0.550 & 0.493 & 0.547 & 0.047 & 0.072 & no \\
\hline $\mathrm{D} 7 \mathrm{~s} 2204$ & Locus 20 & $232-244$ & 5 & -0.016 & 0.686 & 0.676 & 0.165 & 0.007 & 0.009 & no \\
\hline D1s207 & Locus21 & $133-135$ & 2 & -0.087 & 0.464 & 0.479 & 1.000 & -0.016 & -0.024 & no \\
\hline $\mathrm{D} 4 \mathrm{~s} 243$ & Locus22 & $151-163$ & 5 & -0.193 & 0.585 & 0.676 & 0.012 & -0.044 & -0.056 & no \\
\hline D1s548 & Locus 23 & $192-208$ & 5 & 0.052 & 0.710 & 0.592 & 0.015 & 0.064 & 0.083 & yes \\
\hline D21s1142 & Locus24 & $230-242$ & 5 & -0.179 & 0.659 & 0.690 & 0.277 & -0.011 & -0.013 & no \\
\hline \multicolumn{2}{|c|}{ Mean } & - & 3.8 & -0.065 & 0.550 & 0.564 & - & -0.010 & -0.016 & - \\
\hline \multicolumn{2}{|c|}{$\mathrm{SD}$} & - & 1.4 & 0.092 & 0.148 & 0.141 & - & 0.029 & 0.041 & - \\
\hline \multicolumn{2}{|c|}{ Min } & - & 2.0 & -0.231 & 0.262 & 0.282 & - & -0.067 & -0.100 & - \\
\hline \multicolumn{2}{|c|}{ Max } & - & 7.0 & 0.098 & 0.770 & 0.775 & - & 0.064 & 0.083 & - \\
\hline
\end{tabular}

$\mathrm{F}_{\mathrm{IS}}=$ inbreeding coefficient according to Nei (1987); He= expected heterozygosity; Ho= observed heterozygosity; HWE= Hardy-Weinberg equilibrium (* note that $\mathrm{p}$-value was corrected for multiple testing with the Bonferroni adjustment, $\alpha=(0.05 / 24)=0.00208)$; NAFE $=$ null alleles frequencies estimators calculated based on Brookfield (1996) and Chakraborty et al. (1992) and presence of null alleles. (see supplementary appendix 4.3 for details about these calculations). 
Supplementary table S4.7a Descriptive statistics for DSI indices for each time period.

\begin{tabular}{lccc}
\hline & 2014 & 2015 & 2014 and 2015 \\
\hline DSI range & $0.000-12.785$ & $0.000-14.732$ & $0.000-13.437$ \\
DSI median & 0.115 & 0.074 & 0.102 \\
DSI value of top 10\% & 3.605 & 3.232 & 3.539 \\
Total number of dyads & 122 & 114 & 134 \\
Number of dyads above average (DSI > 1) & 26 & 26 & 30 \\
Percentage of dyads above average (DSI > 1) & $21.3 \%$ & $22.8 \%$ & $22.4 \%$ \\
\hline
\end{tabular}

Supplementary table S4.7b Descriptive statistics for bond strength, number of friends and associated secondary males for all males $(n=24)$. Average, standard deviation, minimum and maximum are reported for each behavioral component (mean $\pm \mathrm{SD}$ (min-max)). Note that number of associated secondary males was calculated considering only primary males (see definition in main manuscript) and using averages weighted by the duration of the association in days.

\begin{tabular}{lccc}
\hline & $\mathbf{2 0 1 4}$ & $\mathbf{2 0 1 5}$ & 2014 and 2015 \\
\hline Bond strength (sum of top 3 DSI) & $9.40 \pm 5.08$ & $8.79 \pm 5.26$ & $9.30 \pm 4.56$ \\
& $(1.26-19.37)$ & $(0.67-20.27)$ & $(0.97-16.89)$ \\
Number of friends & $2.26 \pm 1.29$ & $2.36 \pm 2.04$ & $2.50 \pm 1.93$ \\
& $(0-4)$ & $(0-6)$ & $(0-6)$ \\
Number of associated secondary males & $1.67 \pm 1.38$ & $1.70 \pm 1.56$ & $1.65 \pm 1.47$ \\
& $(0.00-4.00)$ & $(0.00-4.25)$ & $(0.00-4.08)$ \\
\hline
\end{tabular}

Supplementary table S4.8a Mean randomized Elo-rating scores (rElo) of 14 male Guinea baboons of party 6 during the study period.

\begin{tabular}{lcccccccc}
\hline Male ID & MLK & JKY & WLD & RDI & TBS & RBT & BEN & ASN \\
rElo & 2.141 & 3.567 & 3.711 & 4.128 & 4.986 & 5.116 & 6.932 & 7.423 \\
\hline Male ID & ASN & LOU & MSA & IND & RGR & CSC & CHR & \\
rElo & 7.423 & 8.933 & 9.232 & 11.022 & 11.377 & 12.732 & 13.700 & \\
\hline
\end{tabular}

Supplementary table S4.8b Mean randomized Elo-rating scores (rElo) of 10 male Guinea baboons of party 9 during the study period.

\begin{tabular}{lllllllllll}
\hline Male ID & DRK & WNT & SNE & AND & MRX & BAA & RCC & HOK & EDM & VNC \\
rElo & 1.230 & 3.136 & 3.679 & 3.988 & 4.256 & 6.131 & 6.974 & 7.648 & 8.836 & 9.122 \\
\hline
\end{tabular}


Supplementary table S4.9 Results of the reproductive skew analysis for parties 6 and 9. Results from the reproductive skew analysis conducted with SKEW CALCULATOR 2013 (10000 permutations). We investigated reproductive skew at the party level using all offspring born in 2014 and 2015 with assigned paternity within-party $(n=18)$ and calculated the Binomial Skew Index $(B$ Obs.), its confidence intervals $(0.25 \% \mathrm{CI}$ and $0.95 \% \mathrm{CI}$ ), the maximum possible $\mathrm{B}$ value (Bmonopoly: reproduction monopolized by one individual), the minimum possible $\mathrm{B}$ value (B-equal: equal sharing among all individuals), and the respective $\mathrm{p}$-values $(\mathrm{P}) . \mathrm{N}$ indicates group size and $\mathrm{Nb}$ the number of individuals that gained at least one reproductive benefit. Values for each party and mean values are reported.

\begin{tabular}{ccccccccc}
\hline Party & $\mathbf{N}$ & $\mathbf{N b}$ & B-equal & $\mathbf{0 . 2 5 \%}$ CI & B Obs. & $\mathbf{0 . 9 5 \%} \mathbf{C I}$ & B-monopoly & P \\
\hline 9 & 9 & 6 & -0.087 & -0.087 & -0.043 & 0.072 & 0.777 & 0.856 \\
6 & 11 & 5 & -0.112 & -0.112 & 0.028 & 0.246 & 0.778 & 0.273 \\
Means & 10 & 5.5 & $/$ & $/$ & -0.010 & $/$ & $/$ & 0.545 \\
\hline
\end{tabular}

Supplementary table S4.10 Results of the paternity analysis for all offspring born during 2014 and 2015 in party 6 and 9. Results from the paternity analysis conducted with Cervus 3.0. The offspring, mother and most likely father identity are reported in the table per each study party. Date (month and year) of conception, birth and identity of the primary male at the time of conception are also reported. Transfer of mother to another primary male's unit between time of conception and birth never occurred (see "Unit transfer during conception/pregnancy"). Nmis indicates number of mismatches; Trio LOD indicates the scores of the logarithm of the likelihood ratio; trio Delta is defined as the difference in LOD scores between the most likely and the second most likely candidate father. The confidence level of the Cervus paternity assignments was set to $95 \%$ ("strict" criterion). An asterisk in the confidence level column indicates a statistical confidence on paternity assignment higher than 95\%. An asterisk in the most likely father column indicates fathers that were not the primary male at the time of conception. In particular, one asterisk indicate a father belonging to the same party and two asterisks a father belonging to a different party of the same gang.

\begin{tabular}{|c|c|c|c|c|c|c|c|c|c|c|c|c|}
\hline Party & $\begin{array}{c}\text { Off- } \\
\text { spring }\end{array}$ & $\begin{array}{l}\text { Time } \\
\text { of } \\
\text { birth }\end{array}$ & $\begin{array}{l}\text { Time } \\
\text { of } \\
\text { Conce- } \\
\text { ption }\end{array}$ & Mother & $\begin{array}{l}\text { Off- } \\
\text { spring } \\
\text { sam- } \\
\text { pling }\end{array}$ & $\begin{array}{l}\text { Most- } \\
\text { likely } \\
\text { father }\end{array}$ & $\begin{array}{c}\text { Primary } \\
\text { male at } \\
\text { time of } \\
\text { conception }\end{array}$ & $\begin{array}{c}\text { Unit } \\
\text { transfer } \\
\text { during } \\
\text { conception } \\
\text { /pregnancy }\end{array}$ & Nmis & $\begin{array}{c}\text { Trio } \\
\text { LOD }\end{array}$ & $\begin{array}{c}\text { Trio } \\
\text { Delta }\end{array}$ & $\begin{array}{l}\text { Conf- } \\
\text { level }\end{array}$ \\
\hline 6 & CRS & Jan-14 & Aug-13 & EKA & Yes & $\mathrm{RDI}^{*}$ & JKY & No & 0 & $1.29 \mathrm{E}+15$ & $1.29 \mathrm{E}+15$ & $95 \% *$ \\
\hline 9 & $\mathrm{NOA}$ & Mar-14 & Oct-13 & IGR & Yes & SNE & SNE & No & 0 & $9.10 \mathrm{E}+14$ & $8.50 \mathrm{E}+14$ & $95 \% *$ \\
\hline 6 & QNN & Apr-14 & Nov-13 & $\mathrm{XNA}$ & Yes & MLK & MLK & No & 1 & 8.69E +14 & $8.69 \mathrm{E}+14$ & $95 \% *$ \\
\hline 6 & $\mathrm{LNO}$ & Jun-14 & Jan-14 & MCY & Yes & JKY & JKY & No & 0 & $9.17 \mathrm{E}+14$ & $9.17 \mathrm{E}+14$ & $95 \% *$ \\
\hline 6 & PTC & Jul-14 & Feb-14 & LCY & Yes & MLK & MLK & No & 0 & $8.52 \mathrm{E}+14$ & $8.52 \mathrm{E}+14$ & $95 \% *$ \\
\hline 9 & $\mathrm{PCO}$ & Jul-14 & Mar-14 & AMT & Yes & BAA & BAA & No & 0 & $1.19 \mathrm{E}+15$ & $1.19 \mathrm{E}+15$ & $95 \% *$ \\
\hline 9 & SPT & Aug-14 & Apr-14 & SND & Yes & DRK & DRK & No & 0 & $9.36 \mathrm{E}+14$ & $9.36 \mathrm{E}+14$ & $95 \% *$ \\
\hline 9 & $\mathrm{HIK}$ & Sep-14 & Mar-14 & GNR & Yes & AND & AND & No & 0 & 7.64E+14 & $7.64 \mathrm{E}+14$ & $95 \% *$ \\
\hline 9 & KTE & Oct-14 & Apr-14 & TBY & Yes & SNE & SNE & No & 1 & $4.80 \mathrm{E}+14$ & $4.80 \mathrm{E}+14$ & $95 \% *$ \\
\hline 6 & SRY & Jan-15 & Sep-14 & DSL & Yes & RBT & RBT & No & 0 & $1.12 \mathrm{E}+15$ & $9.91 \mathrm{E}+14$ & $95 \% *$ \\
\hline 9 & KKI & Feb-15 & Sep-14 & TAR & Yes & DRK & DRK & No & 0 & $6.53 \mathrm{E}+14$ & $6.53 \mathrm{E}+14$ & $95 \% *$ \\
\hline 9 & LLU & Mar-15 & Sep-14 & VTR & Yes & WNT & WNT & No & 0 & $1.30 \mathrm{E}+15$ & $1.30 \mathrm{E}+15$ & $95 \% *$ \\
\hline 9 & SBY & Apr-15 & Nov-14 & KIR & Yes & WNT & WNT & No & 0 & $1.20 \mathrm{E}+15$ & $1.20 \mathrm{E}+15$ & $95 \% *$ \\
\hline 9 & DJO & May-15 & Nov-15 & RXN & Yes & $\mathrm{NDR}^{* *}$ & DRK & No & 0 & $7.27 \mathrm{E}+14$ & $7.27 \mathrm{E}+14$ & $95 \% *$ \\
\hline
\end{tabular}




\begin{tabular}{ccccccccccccc}
6 & BIC & May-15 & Jan-15 & EML & Yes & RBT & RBT & No & 0 & $9.78 \mathrm{E}+14$ & $4.06 \mathrm{E}+14$ & $95 \% \%^{*}$ \\
6 & FIN & May-15 & Nov-14 & SLY & Yes & MLK & MLK & No & 0 & $1.04 \mathrm{E}+15$ & $9.93 \mathrm{E}+14$ & $95 \% \%^{*}$ \\
\hline 6 & MWL & Jun-15 & Feb-15 & LEA & Yes & WLD & WLD & No & 0 & $1.04 \mathrm{E}+15$ & $1.04 \mathrm{E}+15$ & $95 \% \%^{*}$ \\
9 & THL & Aug-15 & Mar-15 & MMI & Yes & MRX & MRX & No & 0 & $9.58 \mathrm{E}+14$ & $3.64 \mathrm{E}+14$ & $95 \% \%^{*}$ \\
9 & LEO & Dec-15 & Jul-15 & DPH & Yes & AND & AND & No & 0 & $6.90 \mathrm{E}+14$ & $6.90 \mathrm{E}+14$ & $95 \% 0^{*}$ \\
6 & EMLi & Sep-14 & Mar-14 & EML & No & $/$ & RBT & No & $/$ & $/$ & $/$ & $/$ \\
9 & OLV & Oct-14 & May-14 & YKO & No & $/$ & MRX & No & $/$ & $/$ & $/$ & $/$ \\
9 & GRM & May-15 & Jan-15 & ELI & No & $/$ & MRX & No & $/$ & $/$ & $/$ & $/$ \\
9 & BMB & Jul-15 & Jan-15 & IGR & No & $/$ & AND & No & $/$ & $/$ & $/$ & $/$ \\
6 & ATAi & Jul-15 & Feb-15 & ATA & No & $/$ & TBS & No & $/$ & $/$ & $/$ \\
\hline
\end{tabular}

Supplementary table S4.11a Model 2a - Kinship and male sociality - Relationship type 1: friend versus non-friend dyads. Model table of male-male relationship type 1's (friend vs. nonfriend dyads) effect on male-male dyadic relatedness estimates

\begin{tabular}{|c|c|c|c|c|c|c|c|}
\hline \multicolumn{8}{|c|}{$\begin{array}{l}\text { Model formula: lmer(Relatedness _wang } \sim \text { RelationshipType1 + Party }+ \\
(1 \mid \text { Male1_ID })+(1 \mid \text { Male2_ID }), \text { data=d, REML=F })\end{array}$} \\
\hline & Estimate & Std. Error & $2.5 \% \mathrm{CI}$ & $97.5 \%$ CI & $x^{2}$ & df & $\operatorname{Pr}$ (Chi) \\
\hline Intercept & 0.216 & 0.044 & 0.127 & 0.303 & (2) & (2) & (2) \\
\hline $\begin{array}{l}\text { Relationship'Type1_Non- } \\
\text { Friends(1) }\end{array}$ & -0.210 & 0.045 & -0.300 & -0.127 & 19.193 & 1 & $<0.001$ \\
\hline Party 9(1) & 0.025 & 0.048 & -0.073 & 0.115 & 0.268 & 1 & 0.604 \\
\hline \multicolumn{8}{|c|}{$\begin{array}{l}\text { Estimates calculated from the generalized linear mixed model with standard errors. Models examine the effects of predictors indicated on the } \\
\text { left on male-male dyadic relatedness estimates (main predictors above the dotted line; control predictors below). CI = confidence interval. } \\
\text { The sample for this model consisted of: number of obs: 134; groups: Male1_ID, 22; Male2_ID, } 22 \\
\text { (1) RelationshipType1 was dummy coded with "NonFriends" being the reference category. Party was dummy coded with Party } 6 \text { being the } \\
\text { reference category. } \\
\text { (2) Not shown due to very limited interpretability. }\end{array}$} \\
\hline
\end{tabular}

Supplementary table S4.11b Model 2b - Kinship and male sociality - Relationship type 2: Primary and their secondary males versus other male-male dyads (i.e. NonPrimarySecondary) Model table of male-male relationship type 2's (Primary and their secondary males vs. other male-male dyads) effects on male-male dyadic relatedness estimates.

\begin{tabular}{|c|c|c|c|c|c|c|c|}
\hline \multicolumn{8}{|c|}{$\begin{array}{l}\text { Model formula: } \\
\text { Lmer(Relatedness_wang } \sim \text { Relationship'Type } 2+\text { Party }+ \\
\qquad(1 \mid \text { Male1_ID })+(1 \mid \text { Male2_ID }), \text { data=d, REML=F })\end{array}$} \\
\hline & Estimate & Std. Error & $2.5 \% \mathrm{CI}$ & $97.5 \% \mathrm{CI}$ & $x^{2}$ & df & $\operatorname{Pr}$ (Chi) \\
\hline Intercept & 0.183 & 0.042 & 0.099 & 0.268 & (2) & (2) & (2) \\
\hline $\begin{array}{l}\text { Relationship'Type2_Non- } \\
\text { PrimarySecondary }{ }^{(1)}\end{array}$ & -0.175 & 0.045 & -0.266 & -0.088 & 12.732 & 1 & $<0.001$ \\
\hline Party 9(1) & 0.024 & 0.047 & -0.066 & 0.112 & 0.247 & 1 & 0.619 \\
\hline \multicolumn{8}{|c|}{$\begin{array}{l}\text { Estimates calculated from the generalized linear mixed model with standard errors. Models examine the effects of predictors indicated on the } \\
\text { left on male-male dyadic relatedness estimates (main predictors above the dotted line; control predictors below). CI = confidence interval. } \\
\text { The sample for this model consisted of: number of obs: } 134 \text {; groups: Male1_ID, 22; Male2_ID, 22 } \\
\text { (1) RelationshipType2 was dummy coded with "NonPrimarySecondary" being the reference category. Party was dummy coded with Party } 6 \\
\text { being the reference category. } \\
\text { (2) Not shown due to very limited interpretability. }\end{array}$} \\
\hline
\end{tabular}


Supplementary table S4.12 Model 3 - Male-male dyadic composite sociality index and coalitionary support. Model table of male-male DSI's effect on coalitionary rate per contact hour.

\begin{tabular}{|c|c|c|c|c|c|c|c|}
\hline \multicolumn{8}{|c|}{$\begin{array}{l}\text { Model formula: } \\
\text { Glmer(Coalition_Count } \sim \text { z.log.DSI + Party }+ \text { Year }+ \\
\quad(1+\text { z.log.DSI + Year_code || Male1_ID })+(1+\text { z.log.DSI + Year_code || Male2_ID }) \\
\quad+(1 \mid \text { Dyad_ID })+\text { offset }(\text { offsetlog_Contact_Hours }), \text { family= "poisson", } \\
\text { data=d, control=contr)contr= glmerControl } \\
\quad(\text { optimizer="bobyqa", optCtrl=list(maxfun=100000) })\end{array}$} \\
\hline & Estimate & Std. Error & $2.5 \% \mathrm{CI}$ & $97.5 \%$ CI & $x^{2}$ & $\mathrm{df}$ & $\operatorname{Pr}(\mathrm{Chi})$ \\
\hline Intercept & -8.645 & 0.301 & -9.270 & -8.105 & (3) & (3) & (3) \\
\hline z.log.DSI(1) & 0.765 & 0.127 & 0.466 & 0.978 & 19.458 & 1 & $<0.001$ \\
\hline Year_2015(2) & 0.293 & 0.332 & -0.371 & 0.951 & 0.585 & 1 & 0.444 \\
\hline Party 9(2) & 1.285 & 0.292 & 0.740 & 1.918 & 12.413 & 1 & $<0.001$ \\
\hline \multicolumn{8}{|c|}{ 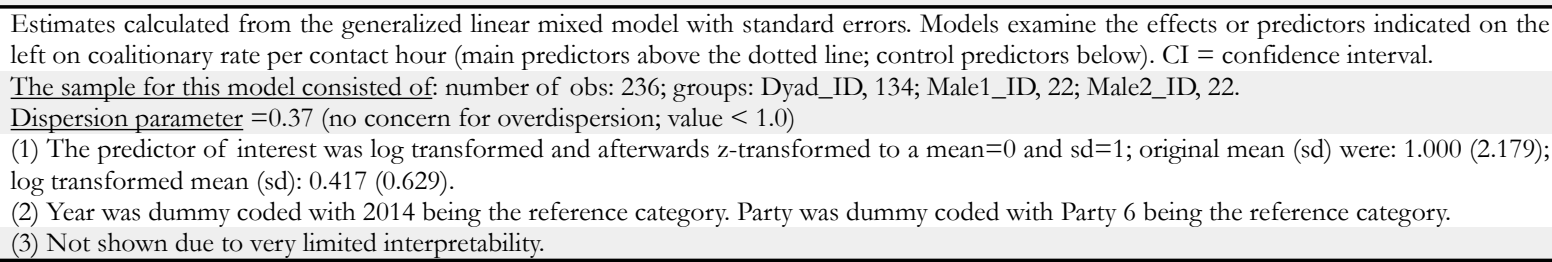 } \\
\hline
\end{tabular}

Supplementary table S4.13a Weighted average of associated females and male sociality Model 4a - Number of friends and bond strength - Model table of male bond strength and number of friends' effect on number of associated females (calculated as average weighted by the duration of the association in days to control for female transfer and demographic changes)

\begin{tabular}{|c|c|c|c|c|c|c|c|}
\hline \multicolumn{8}{|c|}{$\begin{array}{l}\text { Model formula: } \\
\text { Lmer(log.WeightedAverage_Nfemales } \sim \text { z.Bond_Strenght_top3DSI + z.N_Friends + Year + } \\
\text { z.Male_Partner_Availability }+(1 \mid \text { Male_ID }), \text { data }=\text { d, REML=F })\end{array}$} \\
\hline & Estimate & Std. Error & $2.5 \% \mathrm{CI}$ & $97.5 \% \mathrm{CI}$ & $x^{2}$ & $\mathrm{df}$ & $\operatorname{Pr}(\mathrm{Chi})$ \\
\hline Intercept & 0.733 & 0.109 & 0.525 & 0.955 & (3) & (3) & (3) \\
\hline $\begin{array}{l}\text { z.Bond_Strenght_top3- } \\
\text { DSI(1) }\end{array}$ & -0.193 & 0.071 & -0.345 & -0.045 & 6.662 & 1 & 0.010 \\
\hline z.N_Friends ${ }^{(1)}$ & -0.008 & 0.070 & -0.148 & 0.131 & 0.012 & 1 & 0.911 \\
\hline $\begin{array}{l}\text { z.Male_Partner_Availa- } \\
\text { bility(2) }\end{array}$ & -0.137 & 0.103 & -0.326 & 0.074 & 1.738 & 1 & 0.187 \\
\hline Year_2015(2) & -0.090 & 0.081 & -0.254 & 0.063 & 1.190 & 1 & 0.275 \\
\hline \multicolumn{8}{|c|}{ 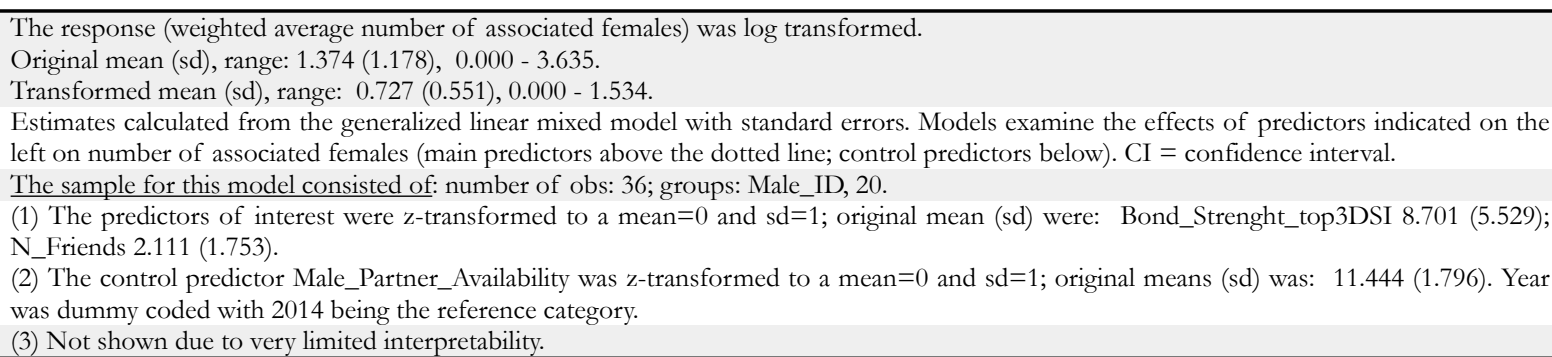 } \\
\hline
\end{tabular}


Supplementary table S4.13b Weighted average of associated females and male sociality Model 4b - Weighted average of secondary males - Model table of number of associated secondary males' effect on number of associated females (calculated as average weighted by the duration of the association in days to control for female transfer and demographic changes)

\begin{tabular}{|c|c|c|c|c|c|c|c|}
\hline $\begin{array}{l}\text { Model formula: } \\
\text { Lmer(log.WeightedAverage } \\
\text { z.Male_Partner_A }\end{array}$ & $\begin{array}{l}\text { emales } ~ \\
\text { sility }+(1\end{array}$ & $\begin{array}{l}\text { Weighted } \\
\text { Male_ID) }\end{array}$ & $\begin{array}{l}\text { erage_N } \\
\mathrm{ta}=\mathrm{d}, \mathrm{R}\end{array}$ & $\begin{array}{l}\text { Males }+ \\
\mathrm{L}=\mathrm{F})\end{array}$ & & & \\
\hline & Estimate & Std. Error & $2.5 \% \mathrm{CI}$ & $97.5 \% \mathrm{CI}$ & $x^{2}$ & df & $\operatorname{Pr}(\mathrm{Chi})$ \\
\hline Intercept & 0.846 & 0.123 & 0.597 & 1.097 & (3) & (3) & (3) \\
\hline $\begin{array}{l}\text { z.WeightedAverage_Nsec- } \\
\text { Males(1) }^{(1)}\end{array}$ & 0.002 & 0.140 & -0.286 & 0.288 & 0.000 & 1 & 0.989 \\
\hline $\begin{array}{l}\text { z.Male_Partner_Availa- } \\
\text { bility(2) }\end{array}$ & -0.139 & 0.142 & -0.414 & 0.147 & 0.927 & 1 & 0.336 \\
\hline Year_2015(2) & -0.062 & 0.106 & -0.252 & 0.153 & 0.343 & 1 & 0.558 \\
\hline 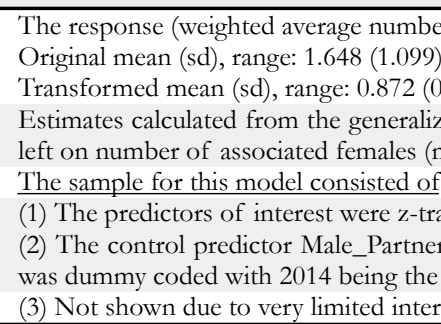 & $\begin{array}{l}\text { issociated fen } \\
7-3.635 . \\
0.007-1.534 . \\
\text { lear mixed } \mathrm{m} \\
\text { redictors abc } \\
\text { ber of obs: } 3 \\
\text { med to a me } \\
\text { ilability was } \\
\text { ence category }\end{array}$ & $\begin{array}{l}\text { el with standa } \\
\text { the dotted lin } \\
\text { groups: Male } \\
=0 \text { and sd }=1 \text {; } \\
\text { ansformed to }\end{array}$ & $\begin{array}{l}\text { ors. Model } \\
\text { trol predic } \\
\text { al mean (sc } \\
\text { an }=0 \text { and }\end{array}$ & $\begin{array}{l}\text { mine the effee } \\
\text { below). } \mathrm{CI}= \\
\text { re: N_Friend } \\
\text {; original mea }\end{array}$ & $\begin{array}{l}\text { ff prec } \\
\text { idence } \\
\text { ing }\end{array}$ & & $\begin{array}{l}\text { ed on the } \\
\text { 845). Year }\end{array}$ \\
\hline
\end{tabular}

Supplementary table S4.14a Number of sired offspring and male sociality - Model 5a Number of friends and bond strength - Model table of male bond strength and number of friends' effect on number of sired offspring

\begin{tabular}{|c|c|c|c|c|c|c|c|}
\hline $\begin{array}{l}\text { Model formula: } \\
\text { Glmer(NsiredOffspring z.B } \\
\text { z.Male_Partner_Availa }\end{array}$ & $\begin{array}{l}\text { d_Strenght } \\
\text { ity }+(1 \mid \mathrm{N}\end{array}$ & $\begin{array}{l}\text { top3DSI + } \\
\text { ale_ID), fam }\end{array}$ & $\begin{array}{l}\text { z.N_Frien } \\
\text { ily= "poiss }\end{array}$ & $\begin{array}{l}\text { ls }+ \text { Year }+ \\
\text { on", data }=\mathrm{d}\end{array}$ & & & \\
\hline & Estimate & Std. Error & $2.5 \% \mathrm{CI}$ & $97.5 \% \mathrm{CI}$ & $x^{2}$ & $\mathrm{df}$ & $\operatorname{Pr}($ Chi $)$ \\
\hline Intercept & -0.612 & 0.336 & -1.717 & -0.153 & (3) & (3) & (3) \\
\hline z.Bond_Strenght_top3DSI(1) & -0.283 & 0.320 & -1.166 & 0.347 & (4) & (4) & (4) \\
\hline z.N_Friends $(1)$ & 0.427 & 0.341 & -0.364 & 1.289 & (4) & (4) & (4) \\
\hline z.Male_Partner_Availability(2) & -0.385 & 0.299 & -1.243 & 0.185 & (4) & (4) & (4) \\
\hline Year_2015(2) & -0.303 & 0.495 & 1.552 & 0.670 & (4) & (4) & (4) \\
\hline $\begin{array}{l}\text { Estimates calculated from the generalized } \\
\text { left on number of associated females (mair }\end{array}$ & nixed model & ith standard err & s. Models ex & ine the effects of & & & ated on the \\
\hline $\begin{array}{l}\text { The sample for this model consisted of: nut } \\
\text { Dispersion parameter }=1.08 \text { (no substanti }\end{array}$ & $\begin{array}{l}r \text { of obs: } 36 ; \mathrm{g} \\
\text { acern for over }\end{array}$ & $\begin{array}{l}\text { ups: Male_ID, } 20 \\
\text { spersion; value }\end{array}$ & & & & & \\
\hline $\begin{array}{l}\text { (1) The predictors of interest were } z \text {-trans } \\
\text { N_Friends } 2.111 \text { (1.753). } \\
\text { (2) The control predictor Male_Partner_Av } \\
\text { was dummy coded with } 2014 \text { being the refe }\end{array}$ & $\begin{array}{l}\text { ned to a mean } \\
\text { bility was z-trat } \\
\text { ce category. }\end{array}$ & $\begin{array}{l}0 \text { and } \mathrm{sd}=1 \text {; origi } \\
\text { formed to a mear }\end{array}$ & $=0$ and $\mathrm{sd}=1$; & $\begin{array}{l}\text { ere: Bond_Stren } \\
\text { griginal means (sc }\end{array}$ & & & $\begin{array}{l}701(5.529) \text {; } \\
1.796) \text {. Year }\end{array}$ \\
\hline $\begin{array}{l}\text { (3) Not shown due to very limited interpret } \\
\text { (4) Not shown as full null model compariso }\end{array}$ & . & $\mathrm{t}(=1.397, \mathrm{df}=$ & $p=0.497)$ & & & & \\
\hline
\end{tabular}


Supplementary table S4.14b Number of sired offspring and male sociality - Model 5b Weighted average of secondary males - Model table of number of associated secondary males' effect on number of associated females (both measures calculated as weighted average of number and association days to control for female transfer and demographic changes)

\begin{tabular}{|c|c|c|c|c|c|c|c|}
\hline \multicolumn{8}{|c|}{$\begin{array}{l}\text { Model formula: } \\
\text { Glmer(NsiredOffspring } \sim \text { z.WeightedAverage_NsecMales + Year + } \\
\quad \text { z.Male_Partner_Availability + }(1 \mid \text { Male_ID }), \text { family= "poisson", data=d })\end{array}$} \\
\hline & Estimate & Std. Error & $2.5 \% \mathrm{CI}$ & $97.5 \% \mathrm{CI}$ & $x^{2}$ & df & $\operatorname{Pr}(\mathrm{Chi})$ \\
\hline Intercept & -0.418 & 0.338 & -1.600 & 0.088 & (3) & (3) & (3) \\
\hline $\begin{array}{l}\text { z.WeightedAverage_Nsec- } \\
\text { Males }^{(1)}\end{array}$ & -0.019 & 0.338 & -0.719 & 0.775 & 0.003 & 1 & 0.955 \\
\hline $\begin{array}{l}\text { z.Male_Partner_Availa- } \\
\text { bility(2) }\end{array}$ & -0.167 & 0.334 & -1.143 & 0.448 & 0.261 & 1 & 0.609 \\
\hline Year_2015(2) & -0.206 & 0.495 & -1.299 & 0.844 & 0.174 & 1 & 0.677 \\
\hline \multicolumn{8}{|c|}{ 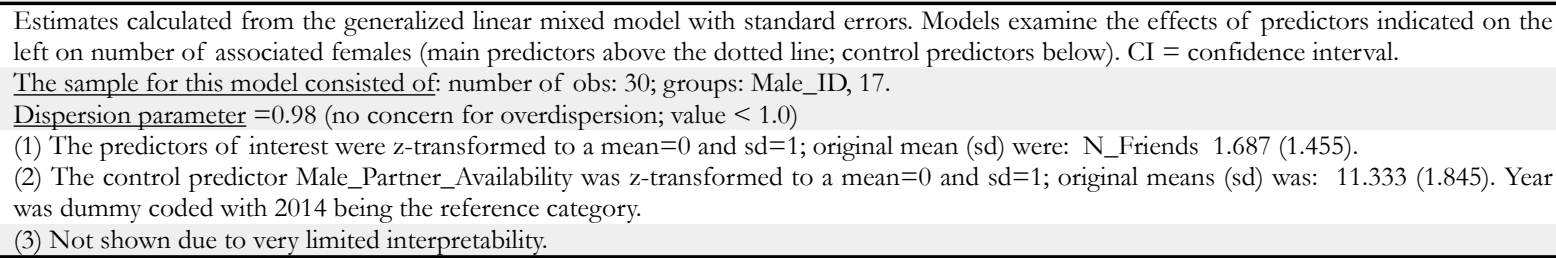 } \\
\hline
\end{tabular}

Supplementary table S4.15 Model 6 - Post-hoc test: time males spent socializing with other males by number of associated females - Model table of focal male's number of associated females' effect on proportion of time spent socializing with other males

\begin{tabular}{|c|c|c|c|c|c|c|c|}
\hline \multicolumn{8}{|c|}{$\begin{array}{l}\text { Model formula: lmer(sqrt.PropTime_Socializing_withOtherMales } \sim \text { z.FocalMaleNfemales + Party } \\
+(1 \mid \text { Male_ID }), \\
\text { data=d, REML=F, control = contr }) \\
\text { contr = lmerControl(optimizer="bobyqa", optCtrl=list }(\operatorname{maxfun}=100000))\end{array}$} \\
\hline & Estimate & Std. Error & $2.5 \% \mathrm{CI}$ & $97.5 \% \mathrm{CI}$ & $x^{2}$ & df & $\operatorname{Pr}(\mathrm{Chi})$ \\
\hline Intercept & 0.140 & 0.016 & 0.107 & 0.171 & (3) & (3) & (3) \\
\hline z.FocalMaleNfemales ${ }^{(1)}$ & -0.042 & 0.011 & -0.064 & -0.019 & 11.932 & 1 & 0.001 \\
\hline Party 9(2) & -0.041 & 0.024 & -0.087 & 0.009 & 2.735 & 1 & 0.098 \\
\hline \multicolumn{8}{|c|}{$\begin{array}{l}\text { The response (proportion of time spent socializing with males) was square-root transformed. } \\
\text { Original mean (sd), range: } 0.022(0.024), 0.000-0.083 \text {. } \\
\text { Transformed mean (sd), range: } 0.120(0.090), 0.000-0.289 \text {. } \\
\text { (1) The predictor of interest was log transformed and afterwards z-transformed to a mean }=0 \text { and sd }=1 \text {; original mean (sd) were: } 1.429 \text { (1.354). } \\
\text { (2) Party was dummy coded with Party } 6 \text { being the reference category. } \\
\text { (3) Not shown due to very limited interpretability. }\end{array}$} \\
\hline
\end{tabular}


Supplementary table S4.16a Male-male association type and interaction/association with primary's associated females - Model 1a - Ad libitum interaction - Model table of male-male association type's (associated vs. non-associated male-male dyads) effect on rate of male ad libitum interactions with primary male's females (rate $=$ count of ad libitum interactions recorded by contact time with the party)

\begin{tabular}{|c|c|c|c|c|c|c|c|}
\hline \multicolumn{8}{|c|}{$\begin{array}{l}\text { Model formula: glmer(Beh_adlib_Count } \sim \text { MMAssociationType }+ \text { Party }+ \\
\text { z.Female_number_perPeriod }+ \\
(1+\text { Male_Type_MMasso_code }+ \text { z.FemaleNumberPerPeriod || Male_ID })+ \\
(1+\text { Male_Type_MMasso_code || Primary_Male_ID })+(1 \mid \text { Dyad_ID })+ \\
\text { offset(offsetlog_Contact_Hours }), \\
\text { data=d, family="poisson", control = contr }) \\
\text { contr=glmerControl(optimizer="bobyqa", optCtrl=list }(\operatorname{maxfun=100000)})\end{array}$} \\
\hline & Estimate & Std. Error & $2.5 \% \mathrm{CI}$ & $97.5 \%$ CI & $x^{2}$ & df & $\operatorname{Pr}($ Chi) \\
\hline Intercept & -4.161 & 0.424 & -5.014 & -3.341 & (3) & (3) & (3) \\
\hline $\begin{array}{l}\text { MMAssociationType_Not- } \\
\text { Associated(1) }\end{array}$ & -3.227 & 0.397 & -4.017 & -2.360 & 22.736 & 1 & $<0.001$ \\
\hline Party 9(1) & -0.443 & 0.577 & -1.624 & 0.675 & 0.561 & 1 & 0.454 \\
\hline $\begin{array}{l}\text { z.FemaleNumberPer- } \\
\text { Period(2) }\end{array}$ & 0.881 & 0.225 & 0.440 & 1.281 & 11.402 & 1 & 0.001 \\
\hline \multicolumn{8}{|c|}{ 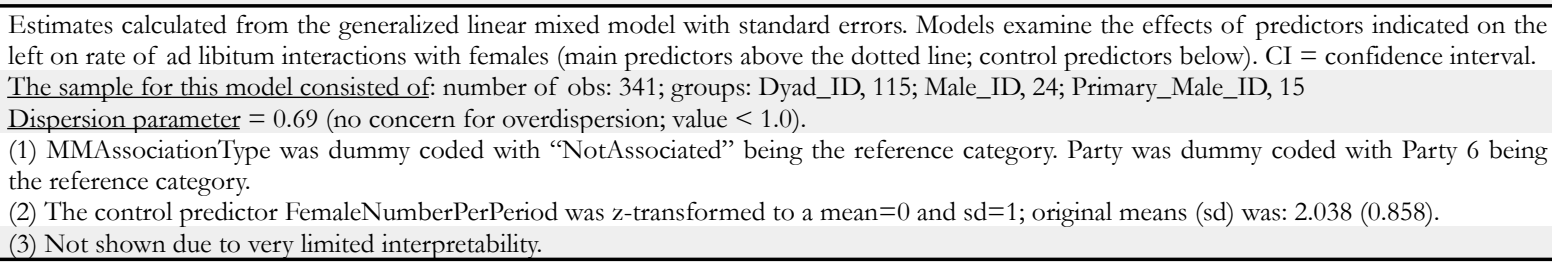 } \\
\hline
\end{tabular}

Supplementary table S4.16b Male-male association type and interaction/association with primary's associated females - Model $1 \mathrm{~b}$ - Focal interactions - Model table of male-male association type's (associated vs. non-associated male-male dyads) effect on rate of male focal interactions with primary male's females (rate $=$ count of focal interactions recorded by focal duration)

\begin{tabular}{|c|c|c|c|c|c|c|c|}
\hline \multicolumn{8}{|c|}{ 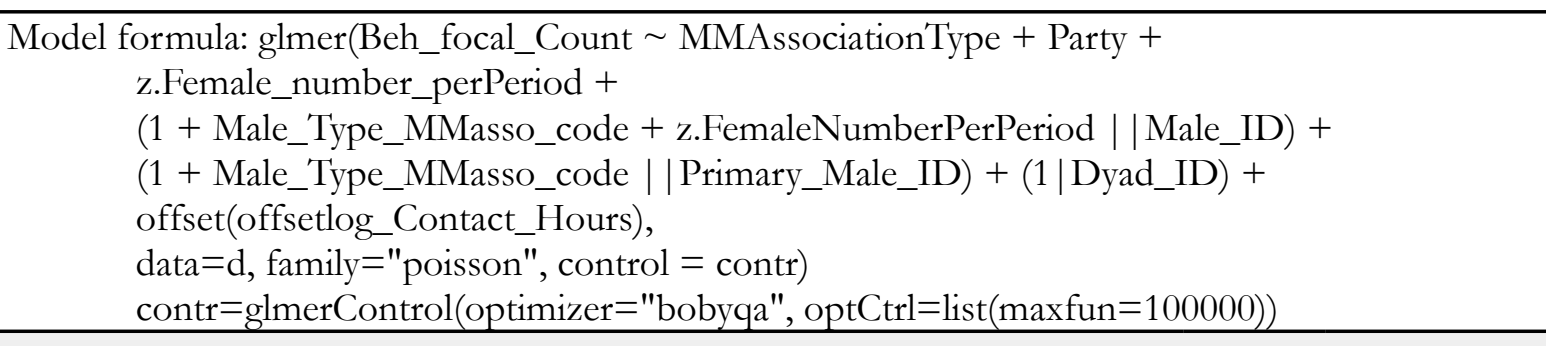 } \\
\hline & Estimate & Std. Error & $2.5 \% \mathrm{CI}$ & $97.5 \%$ CI & $x^{2}$ & $\mathrm{df}$ & $\operatorname{Pr}(\mathrm{Chi})$ \\
\hline Interc & -2.057 & 0.444 & -2.919 & -1.276 & (3) & (3) & (3) \\
\hline $\begin{array}{l}\text { MMAssociationType_Not- } \\
\text { Associated }^{(1)}\end{array}$ & -3.674 & 0.433 & -4.526 & -2.822 & 31.205 & 1 & $<0.001$ \\
\hline Party 9(1) & 0.124 & 0.620 & -1.060 & 1.332 & 0.040 & 1 & 0.842 \\
\hline $\begin{array}{l}\text { z.FemaleNumberPer- } \\
\text { Period(2) }\end{array}$ & 0.858 & 0.194 & 0.497 & 1.190 & 14.212 & 1 & $<0.001$ \\
\hline \multicolumn{8}{|c|}{ 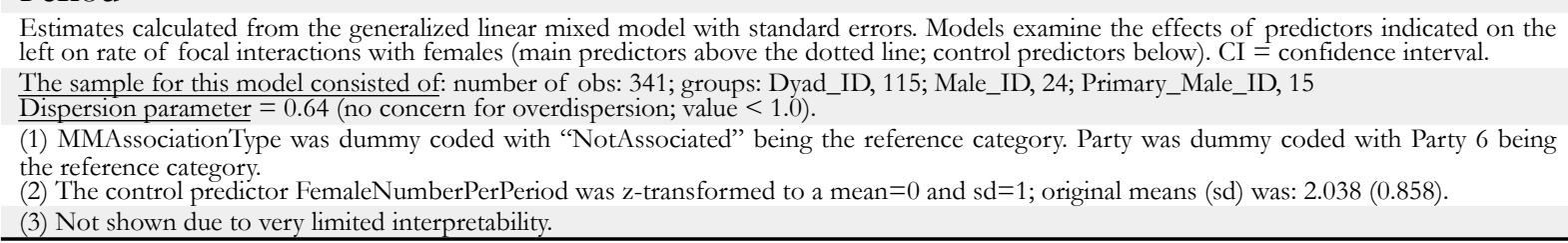 } \\
\hline
\end{tabular}


Supplementary table S4.16c Male-male association type and interaction/association with primary's associated females - Model 1c - Proportion of time spent in 1m proximity - Model table of male-male association type's (associated vs. non-associated male-male dyads) effect on proportion of time males spent within $1 \mathrm{~m}$ proximity to the primary male's females (proportion $=$ count of time recorded within $1 \mathrm{~m}$ by total number of scans)

\begin{tabular}{|c|c|c|c|c|c|c|c|}
\hline \multicolumn{8}{|c|}{ 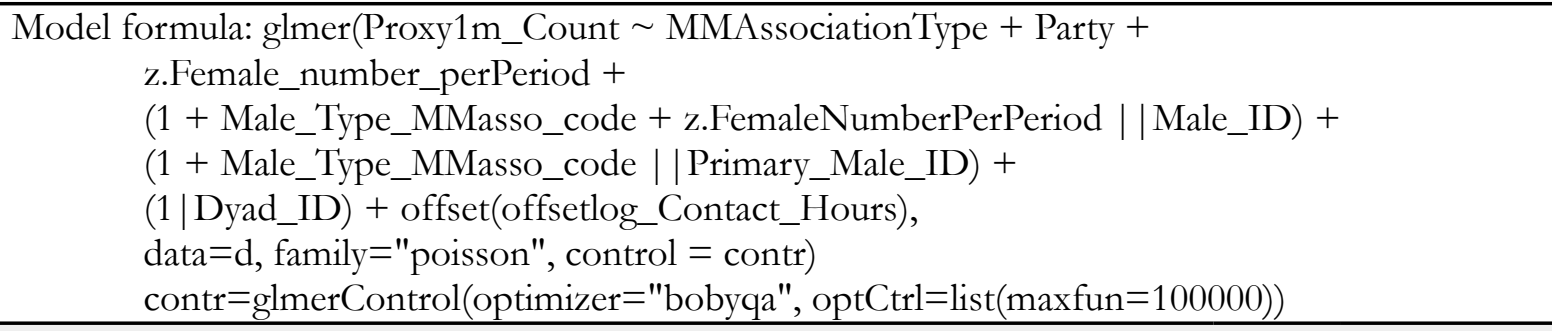 } \\
\hline & Estimate & Std. Error & $2.5 \% \mathrm{CI}$ & $97.5 \%$ CI & $x^{2}$ & df & $\operatorname{Pr}($ Chi $)$ \\
\hline Intercept & -4.233 & 0.248 & -4.770 & -3.763 & (3) & (3) & (3) \\
\hline $\begin{array}{l}\text { MMAssociationType_Not- } \\
\text { Associated(1) }\end{array}$ & -2.919 & 0.316 & -3.497 & -2.281 & 27.901 & 1 & $<0.001$ \\
\hline Party 9(1) & 0.406 & 0.318 & -0.187 & 0.973 & 1.596 & 1 & 0.206 \\
\hline $\begin{array}{l}\text { z.FemaleNumberPer- } \\
\text { Period(2) }^{(2)}\end{array}$ & 0.698 & 0.101 & 0.494 & 0.883 & 30.148 & 1 & $<0.001$ \\
\hline \multicolumn{8}{|c|}{ 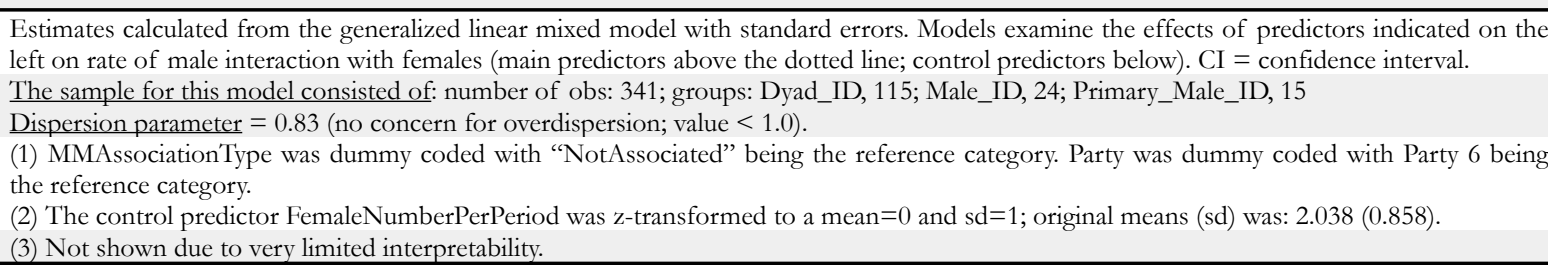 } \\
\hline
\end{tabular}




\section{General Discussion}

This thesis aims to elucidate the dynamics and fitness benefits of male-male sociality in wild Guinea baboons as well as the function of ritualized greeting behavior. Guinea baboon males display affiliative relationships and cooperation, while females have relatively high leverage in intersexual relationships (Fischer et al., 2017). Combined with high levels of male-male spatial tolerance, low aggression and lack of a clear dominance hierarchy, the unique characteristics of Guinea baboons make them an intriguing model to investigate how co-resident males regulate their relationships and how the advantages of male-male sociality play out in a highly tolerant multilevel system.

\subsection{Summary of results}

In chapter 3, I show ritualized greetings are the most common wild Guinea baboon male interaction and describe how they serve an important function in regulating male relationships. Greetings occur at all levels of the society but, controlling for partner availability, males that belong to the same party exchange significantly more greetings than males that belong to different parties. I also describe the function of within-party greetings, the lack of support for the tension-buffering hypothesis, and that dyads do not greet due to simply being in close proximity immediately prior to a greeting. Instead, greetings are used by males to assess the quality of relationships with spatially tolerant partners. Although affiliation does not predict greeting rate, intense greetings (i.e. the most intimate and risky form of greetings) are more likely between males with stronger affiliative relationships. In chapter 4, I describe within-party male-male social relationships and their adaptive value. I demonstrate that wild Guinea baboon males form strong social bonds, and that strongly bonded males are more likely to support each other in coalitions and have a higher average degree of relatedness. I corroborate previous findings that adult males have no clear linear dominance hierarchy (also see chapter 3) and, using recent methodologies, find this is due to intermediate/low steepness, high variation and the uncertainty of individual rank scores. Paternity data shows the share of reproduction at the party level is not significantly different from chance, while at the unit level $89.5 \%$ of offspring are sired by the primary male. Contrary to findings in other species, male-male sociality does not positively affect either number of associated females or sired offspring. Instead, males with stronger bonds have fewer associated females and it may be that time constraints affect relationship investment. 


\subsection{Ritualized greeting behavior in a tolerant multilevel society}

Greeting behavior is crucial when balancing the trade-offs imposed by male co-residence (Whitham and Maestripieri, 2003). These ritualized exchanges are hypothesized to buffer tension during competitive contexts (Colmenares et al., 2000; Aureli and Schaffner, 2007), signal dominance status (Colmenares, 1990, 1991b), and/or function as honest signals to test relationship quality, maintain social bonds, and promote cooperation (Smuts and Watanabe, 1990; Whitham and Maestripieri, 2003; De Marco et al., 2014). The features and functions of these interactions vary considerably between and within species and remain disputed in several cases (see section 2.5.3). The tenor of male-male relationships and levels of tolerance and cooperation may influence this variation (Henzi et al., 2008; De Marco et al., 2014; De Marco, 2017b). I contribute to this debate through my findings on greeting behavior in wild Guinea baboons (chapter 3), which are characterized by an intense and highly ritualized greeting repertoire involving potentially harmful behaviors such as genital fondling.

Greetings occur at all levels of the multilevel society but, controlling for partner availability, their occurrence decreases significantly from the inner-most to outer level of the society. Greetings delineate social levels and party membership, as the vast majority of greetings (94.6\%) occur within the same party, even when other parties and gangs are adjacent or intermingled (chapter 3). Ritualized greetings are also the most common interactions between males, occurring twice as often as affiliation and ten times more than aggression. In accordance with the tolerant style of this species, and in contrast to hamadryas baboons (Colmenares et al., 2000), there is no support for tension buffering. Greeting occurrence is not temporally associated with tense contexts or the presence of receptive females, and males with higher levels of dyadic aggression do not greet at a higher rate (chapter 3). The absence of a dominance hierarchy and lack of strong directionalities in greetings show these ritualized exchanges are unlikely to express social status. This is corroborated by Vasey and Sommer (2006), who state that dominance expression is unlikely to apply to species with a highly symmetrical greeting repertoire.

Wild Guinea baboon ritualized greetings are friendly interactions that allow males to assess relationship quality independent of context, confirming findings in species characterized by male cooperation (Smuts and Watanabe, 1990; Whitham and Maestripieri, 2003). Similar to tolerant Tonkean macaques (De Marco et al., 2014), male Guinea baboons greet specific individuals to assess relationship quality with spatially tolerant partners (chapter 3). Intense and potentially costly greetings between strongly bonded males likely play a role in the assessment and maintenance of relationship strength (chapter 3; also see Whitham and Maestripieri, 2003). My findings regarding context and function nicely mirror the features of greetings in Guinea baboons. Most greetings are reciprocated $(81.9 \%)$, involve physical contact $(93.4 \%)$, and are intense $(59.2 \%)$. Their positive tenor is in sharp contrast to those described for other species of this genus (chapter 3), where greetings are absent, 
incomplete, include little physical contact, and may even be associated with submissive behavior (e.g. chacma baboons: Henzi et al., 2008; yellow baboons: Hausfater and Takacs, 1987; hamadryas baboons: Kummer, 1968; Abegglen, 1984; Colmenares, 1990; olive baboons: Smuts and Watanabe, 1990).

My results show how ritualized greetings allow male Guinea baboons to regulate social dynamics by signaling party membership, testing relationships between spatially tolerant partners, and accentuating bond strength. Individuals of the same party associate frequently and share feeding patches, traveling routes, and sleeping trees (Fischer et al., 2017). Thus, mechanisms to ensure relationship regulation are crucial in reducing the costs of intra-group competition, while increasing group cohesion. This may be especially important in wild Guinea baboons due to their large communities, where individuals encounter and mingle with less familiar individuals of other parties, gangs, or even unfamiliar individuals from neighboring communities. A brief "honest communicative exchange" (Whitham and Maestripieri, 2003) to test the relationship state may be crucial in such a complex environment. Whether ritualized behaviors make a more tolerant social style possible, are an outcome of more cooperative and tolerant social systems, or whether these two features coevolved remains an open question and an interesting direction for future research.

\subsection{The tolerant baboon: male-male sociality, kinship, and competition}

\subsubsection{Male-male sociality and kinship}

My project tests for the presence of male social bonds (Silk, 2002) in Guinea baboon societies for the first time and expands our understanding of the role of kinship (chapter 4). Patzelt et al. (20014) investigated general patterns of male-male sociality in the same population but focused on male-male relationships at different levels of the society and could not consider male status (primary/secondary males) as male-female associations were not known at the time. Here, I focus on the party level, our group unit (Dal Pesco \& Fischer, 2018), and provide data on all males of each party while considering male status. Affiliative relationships between males at the party level are differentiated and preferred partners are stable over time, which shows that Guinea baboon males form strong social bonds (i.e. "friendships"). Primary males form strong bonds with other primaries and with non-primary males that are associated with their unit (i.e. "secondary males"). As strong bonds even occur between nonprimary males, friendships are not restricted to any particular kind of dyad (chapter 4). Strongly bonded dyads support each other more often during within-party aggressive events and average relatedness is higher for friends and for dyads composed of primary males and their secondary males (chapter 4). Corroborating previous findings (Patzelt et al., 2014), males exhibit high levels of withinparty spatial tolerance with $67.9 \%$ and $98.5 \%$ of dyads recorded at least once within $1 \mathrm{~m}$ and $5 \mathrm{~m}$ proximity, respectively (chapter 3). The presence of multi-male units is substantial (chapter 4), with 
most primary males $(76.5 \%)$ associated with at least one secondary male and more than half of these with more than one (61.5\%; average number secondaries per unit=1.65). Remarkably, secondary males have stable associations with multiple units, unaffiliated males (termed "solitary" in hamadryas baboons) are rare, and there are no reports of bachelor groups (chapter 4; also see Goffe et al., 2016). My findings highlight the fluidity of Guinea baboon society through their numerous and dynamic inter-unit social ties and spatial tolerance, while strengthening the view that parties are at the core of these societies (see chapters 3 and 4 ).

Male Guinea baboon sociality sets them apart from multilevel societies in geladas and hamadryas baboons. Gelada unit leaders usually ignore each other except for rare support of females during interunit disputes (Dunbar, 1983). Multi-male units occur only in 33\% of cases (Snyder-Mackler et al., 2012; also discussed in Chowdhury et al., 2015) and affiliation between leaders and followers is rare, with followers often at the periphery of units (Dunbar and Dunbar, 1975b). While leaders are sometimes reported jointly confronting bachelor male groups (Dunbar and Dunbar, 1975a), gelada units seem to constitute distinct social entities (Bergman, 2010) within loosely associated "bands". In hamadryas baboons, several units comprise a "clan" together with solitary males (Abegglen, 1984; Schreier and Swedell, 2009). While occasional alliances occur during inter-clan conflicts (Abegglen, 1984), inter-unit interactions are sporadic and tend to be limited to threats and avoidance between leaders (Kummer, 1968). Leaders never affiliate with other leaders but may groom their unit followers (Schreier and Swedell, 2009; Chowdhury et al., 2015). Solitary males are not associated with a unit and may exchange grooming with other solitary males (Abegglen, 1984). The occurrence of multi-male units $(55.4 \%)$ as well as the proportion of units with more than one secondary male $(38.9 \%$; average number secondaries per unit $=0.80$ ) is lower compared to my findings for Guinea baboons (Chowdhury et al., 2015). This is particularly striking considering that I used a much stricter spatial criterion to identify secondary males $(1 \mathrm{~m})$ than that used in hamadryas baboon studies $(5 \mathrm{~m}$ ) (Chowdhury et al., 2015). Hamadryas follower males are also mainly associated with a particular unit and further associations with other units are rare, short-term, and do not extend to females (Chowdhury et al., 2015). In contrast, secondary males in Guinea baboons have multiple associations that can last several years and involve interactions with primary males and their associated females (chapter 4).

The striking difference between geladas and Guinea baboons is not surprising given that gelada females are philopatric and units are based on strong bonds between related females (Dunbar, 1983; le Roux et al., 2011). Hamadryas and Guinea baboon societies are characterized by female-biased dispersal (Kopp et al., 2015; Städele et al., 2015) with strong bonds between related males hypothesized as the reason for the structure of these societies (Kummer, 1968; Abegglen, 1984; reviewed in Grueter et al., 2012). Indeed recent field studies on hamadryas baboons show evidence for male philopatry at the clan level and maternal kin biases between leader and their follower males (Städele et al., 2015, 2016; also see Colmenares, 1992). A previous analysis in wild Guinea baboons, however, 
revealed no correlation between affiliation and relatedness at the gang level (Patzelt et al., 2014). With a larger dataset, with all adolescent/adult males of each study party included, I show that average relatedness is significantly higher between friends and between primary males and their secondary males within the party (chapter 4). The fact that relatedness is similar within and between parties of the same gang, but affiliation is significantly higher within parties (Patzelt et al., 2014) may explain the differences between these findings. I was able to confirm that kinship likely played a crucial role in the evolution of male-male social bonds.

However, while kinship influences some bonds, others are independent of kinship (chapter 4). Strongly bonded but weakly related males in our population may be due to lack of available kin. The low reproductive skew at the party level (chapter 4) and potentially low average relatedness for the philopatric sex in large groups (Lukas et al., 2005) support this possibility. However, the presence of weakly bonded males with a high likelihood of relatedness (chapter 4) suggest other mechanisms play a role. As both males and females care for young (personal observation), father- and mother-mediated familiarity may play a role in bond formation (Widdig, 2007; Smith, 2014). At the unit level, mate fidelity is high (89.5\% of offspring sired by the primary, chapter 4$)$, indicating that within-unit peers are likely to be paternal kin. These mechanisms may work within the unit, but not at the party level due to low reproductive skew (chapter 4) and the minor role of kinship in female associations (Goffe, 2016). This ambiguity is compounded by several factors, namely female transfers with dependent offspring between and within social levels and the lack of infanticide (Goffe et al., 2016). Thus, relatedness and familiarity do not mirror each other exactly and lead to a more complex scenario. If familiarity is the major mechanism of kin discrimination in Guinea baboons, the partial overlap between male relatedness and sociality can be explained by unit composition changes during early development. Indeed, reports from a captive population suggest that male-male associations emerge early in life (Boese, 1975), and studies on hamadryas multilevel societies suggest familiarity is associated with the formation and reinforcement of groups and subgroups (Kummer et al., 1974). Familiarity may even confer important fitness benefits irrespective of kinship, as seen in female house mice (Koenig, 1994) and vampire bats where non-kin expand the network of potential future food donors and help cope with the loss of a partner (Desmodus rotundus: Carter and Wilkinson, 2015; Carter et al., 2017). Ties with non-kin may be crucial in dealing with changes in the social environment, especially if support from kin is uncertain. Thus, kinship may facilitate the development of cooperation and bonds through inclusive fitness benefits, but is not the only driving force nor a necessary precondition in Guinea baboons (Fischer et al., 2017). This parallels evidence from others mammals where male bonds are not restricted to kin (Krutzen et al., 2003; Langergraber et al., 2007, 2009; Schülke et al., 2010; Hirsch et al., 2013; Best et al., 2014). 


\subsubsection{Competition and dominance hierarchy}

Adult male Guinea baboons have lower aggression rates than chacma baboons and no clear linear dominance hierarchy (Patzelt et al., 2014; Kalbitzer et al., 2015). In this thesis I concentrate on within-party relationships and include data on all adult and adolescent males in those parties. Using this larger dataset, I apply similar (chapter 3) as well as novel methodologies (chapter 4) to assess male dominance hierarchies in our population. My study confirms past findings of low aggression, supplants, and unprovoked submissions and the limits to establishing a clear linear hierarchy for adult males (chapter 3). Observations of a captive population of Guinea baboons supports this as well, as dominance relationships between adult males could not be clearly identified (Whitham and Maestripieri, 2003).

I can present novel evidence for significant linear hierarchies once males of all age classes (i.e. large juveniles and small/large subadults) are included in the analysis. While the larger sample size may play a role, younger individuals consistently ranked below adult males, which indicates this is likely driven by an effect of age (chapter 3). I additionally apply a recently published approach (Sánchez-Tójar et al., 2018) to assess hierarchy steepness and uncertainty and calculate randomized Elo-rating scores (see details in chapter 4). I found that male hierarchies are of intermediate/low steepness with high intra-individual variation in randomized Elo-rating scores and great overlap between the $95 \%$ score ranges for most males (chapter 4). Hierarchy uncertainty tests (Sánchez-Tójar et al., 2018) demonstrate that estimates are uncertain and, consequently, that dominance cannot be included as a predictor in the analyses (chapter 4). The uncertainty is driven by both the low rate of aggression and high proportions of polyadic and undecided interactions, which leads to a limited number of interactions available for dominance assessments (see details in chapter 4).

The absence of a dominance hierarchy enforced through agonistic interactions shows the limited role agonistic competition and rank play in male Guinea baboons (also see Patzelt et al., 2014; Kalbitzer et al., 2015). This is not surprising considering access to females is achieved through stable pair bonds that allow primary males to monopolize $98.6 \%$ of copulations (Goffe et al., 2016) and sire $89.5 \%$ of offspring within their units (chapter 4). Limited roles for contest competition and sperm competition are supported by the reduced sexual dimorphism compared to savannah baboons and relatively small testis size compared to other species in this genus (Fischer et al., 2017). Although the lack of dominance hierarchy is striking, similar patterns are also reported for other multilevel societies. In these cases male status replaces dominance rank and leaders are considered dominant over non-leaders, who tend to be younger males or older ex-leaders (e.g. Colmenares, 1990; Bergman et al., 2009). Signals of male status other than rank may play a role in signaling "male condition and stamina". Male geladas with redder chest patches have higher status and larger units (Bergman et al., 2009), while mane coloration, size and hind-quarter coloration are proposed as honest signals in hamadryas and Guinea baboons (Kummer, 1997; Jolly, 2007; Kalbitzer, 2014; Fischer et al., 2017). Similar to our 
population, Kummer (1997; p. 166-168) explains hamadryas males lack a rank order, mutually back down from confrontations, and rarely display submissive signals. Their greetings often involve hindquarter presentations and function as a negotiation mechanism to signal competitive power and diffuse tension in contexts of rivalry over females (Abegglen, 1984; Kummer et al., 1974; Colmenares, 1991b; Colmenares et al., 2000; note that Colmenares's investigation focused on a captive population of hamadryas baboons, yellow baboons, and their hybrids). With their more tolerant social style Guinea baboons exchange intense greetings that often involve mutual physical contacts and are not related to competition or aggression (chapter 3, section 5.2, also see De Marco et al. 2014; De Marco 2017). The high proportion of undecided fights (chapter 4) may also be linked to the more tolerant social style (Palagi, 2006; Ciani et al., 2012).

\subsection{The adaptive benefits of male-male sociality}

Although multi-male associations and male bonds are puzzling due to competition over un-sharable fertilization (van Hooff, 2000), recent findings show these relationships can emerge as a result of increased between-group competition and reduced within-group contest potential and may confer substantial fitness benefits (Ostner and Schülke, 2014). In several mammal species, multi-male alliances and rank-changing coalitions between strongly bonded males are linked with lower takeover likelihoods and/or higher male reproductive success (Feh, 1999; Fedigan et al., 2004; Ostner and Kappeler, 2004; Schülke et al., 2010; Snyder-Mackler et al., 2012; Wiszniewski et al., 2012; Gilby et al., 2013; Chowdhury et al., 2015). Additionally, male reproductive success can also be affected by female mate choice (Kulik et al., 2012; Massen and Vries, 2012; Ostner et al., 2013; Arseneau et al., 2015; Rosenbaum et al., 2018).

In geladas and hamadryas baboons, leader males benefit from follower males associated with the unit through increased tenure length, number of females or number of offspring (Snyder-Mackler et al., 2012; Chowdhury et al., 2015). In horses, dominant stallions that fail to establish their own group form stable male pair alliances that aid in joint female protection and result in increased access to females and higher offspring survival (Feh, 1999). Similar to the importance of cooperative defense in horses due to year-round challenges (Feh, 1999), followers in geladas and hamadryas baboons reduce the likelihood of unit takeovers, which otherwise lead to male acquisition of females and infanticide (Swedell and Tesfaye, 2003; Beehner and Bergman, 2008; Pappano et al., 2012; Snyder-Mackler et al., 2012; Swedell et al., 2014; Pines et al., 2015). The associated males, in geladas and horses, benefit from a small proportion of reproduction in the unit (Feh, 1999; Snyder-Mackler et al., 2012).

My results, however, indicate that these patterns do not apply to wild Guinea baboon male reproductive success, at least in the short-term. The number of associated secondary males is not linked to a higher number of associated females or sired offspring (chapter 4). While exceptional, these findings may simply reflect the fluidity and tolerance of this social system. Secondary males may not be as 
crucial to unit defense, as takeovers are rare (Goffe et al., 2016; Fischer et al., 2017) and male competition is low (Patzelt et al., 2014; Kalbitzer et al., 2015; Fischer et al., 2017). Most units also have several associated secondary males and secondary males are shared by multiple units (chapter 4), all of which leaves little room for variation in reproductive success. Moreover, the fact that secondaries are associated with multiple units implies that, during challenges between primary males, associated males do not directly translate to unconditional support. I also show primary male paternity certainty is high and that the remaining $10.5 \%$ of offspring during the study are sired by both a subadult male of the same party and an adult male from a different party (chapter 4). The fact that paternity is not restricted to the unit indicates concessions by the primary male are unlikely, and monopolization is likely limited by other factors such as unit cohesion, reproductive synchrony, and visibility (van Schaik and van Hooff, 1983; Rowell, 1988; Ostner et al., 2008b; Carnes et al., 2011). In Guinea baboons these limiting factors are intensified by the high level of female spatial freedom and their active role in intersexual relationships, as well as the extremely poor visibility during the rainy season (Patzelt, 2013; Goffe et al., 2016).

Male reproductive success can also be affected by male-male social bonds and differentiated withingroup coalitionary support. The adaptive value of cooperation between strongly bonded males is often attributed to their role in rank ascension and consolidation that ultimately leads to increased reproduction (e.g. Schülke et al., 2010). This kind of male cooperation is thought to evolve in scenarios of medium to low contest potential and is reported for both philopatric and dispersing males (e.g. Schülke et al., 2010; Gilby et al., 2013; reviewed in Ostner and Schülke, 2014). Nevertheless, the viability of this strategy depends on male time budget and mating strategies, including year-round breeding and pair bonds (Ostner and Schülke, 2014). I show that in wild Guinea baboons, within-party coalitionary support is present both within and between units (chapter 4). Parallel to findings in macaques, where affiliative relationships predict levels of male cooperation (Silk, 1994; Schülke et al., 2010; Berghänel et al., 2011a; Young et al., 2014b), I found that males with stronger bonds support each other more often during aggressive events. However, rates of coalitionary support are surprisingly low compared to macaque species (Guinea baboons: 0.002/hr; Assamese macaques: 0.11/hr; Barbary macaques: 0.01-0.21/hr; Schülke et al., 2010; Berghänel et al., 2011a) and mirror the low aggression rate (Kalbitzer et al., 2015). Stable pair bonds (Goffe et al., 2016), non-seasonal reproduction (Boese, 1973), and the lack of rank (chapter 3 and 4, section 5.3.2), suggest rank-changing coalitions are not as profitable and may also be limited by male time budget constraints (discussed in Ostner and Schülke, 2014). Indeed, while strongly bonded males cooperate more (chapter 4), bond strength and number of friends are not positively linked with number of associated females or sired offspring (chapter 4). Though my study is limited to two years, this study duration is equal to the case of Schülke and colleagues (2010), who show direct fitness benefits in male Assamese macaques. Compared to other species, my results show sociality need not be directly linked to increased short-term 
reproductive success, though why male Guinea baboons engage in coalitions, and whether these confer benefits in the long-term, remains unclear.

Males are thought to exclusively compete over fertilization, while females compete over other resources, but a recent review points out that this dichotomy is overly simplistic ( $\mathrm{Li}$ and Kokko, 2019). Food and other resources may limit females as well as males, and females may also compete over access to mates ( $\mathrm{Li}$ and Kokko, 2019). Strong bonds and cooperation can benefit males beyond reproductive success. Male-male social bonds may promote efficient cooperation and coordination (Melis et al., 2006; Massen et al., 2010), which are key to more fluid societies where males cooperate within parties as well as across social levels (Patzelt, 2013; Patzelt et al., 2014). Strong bonds and sociability are linked to anti-predator behavior and stronger responses to recruitment alarm calls (Micheletta et al., 2012; Josephs et al., 2016; Kern and Radford, 2016) and efficient cooperation is crucial during cooperative hunting (Drea and Carter, 2009; Samuni et al., 2018a). In fact, male Guinea baboons cooperate in predator defense and chase predators away in groups (Patzelt, 2013 personal observation), and cooperation may increase tolerance during feeding (Tiddi et al., 2011; Dubuc et al., 2012) and meat sharing (Wittig et al., 2014; Goffe and Fischer, 2016; Samuni et al., 2018b). Enhanced agonistic support can result in decreased risk of injuries (Ostner and Schülke, 2018 p. 147), which would explain the rarity of injuries in this species (Kalbitzer et al., 2015). Overall, male-male sociability may reduce aggression and its costs, and result in more time available for pair bonds, which are not maintained by coercion (Fischer et al., 2017).

\subsection{The female perspective and the influential role of pair bonds}

In species characterized by some degree of female choice, females may prefer males with strong alliances as this could reduce harassment from other males and infanticide (Smuts and Smuts, 1993; Rubenstein, 1994; Snyder-Mackler et al., 2012; Chowdhury et al., 2015). However, while Guinea baboons present a degree of female leverage in intersexual relationships (Fischer et al., 2017), my results show that males with more friends and higher bond strength and, consequently, higher levels of support (see above) are not associated with more females (chapter 4). Unlike geladas or hamadryas baboons (Snyder-Mackler et al., 2012; Chowdhury et al., 2015), females may not gain much from preferring males with strong bond, which can positively influence takeover outcomes (Altmann, 1990; Smuts and Smuts, 1993), as competition is low, takeover occurrence is rare, and infanticide is nonexistent (Kalbitzer et al., 2015; Goffe et al., 2016). Females may instead prefer males that provide higher levels of affiliation and spatial freedom (Goffe, 2016) or, as recently reported in mountain gorillas (Rosenbaum et al., 2018), males that care more for offspring. Males also frequently intervene in agonistic disputes to support their females (Faraut \& Dal Pesco, personal observation). Males that provide such support may also be favored, as reported for rhesus macaques and vervet monkeys (Kulik et al., 2012; Arseneau et al., 2015). 
One of the most interesting findings from my thesis is that males with stronger bonds have significantly lower numbers of associated females, instead of having a positive effect on short-term male reproductive success (chapter 4). Through a post-hoc analysis I additionally show that males with more associated females actually spend a significantly lower proportion of time socializing (grooming and contact-sitting) with other males (chapter 4). Guinea baboon males appear to face a trade-off between investments in same-sex bonds and heterosexual ones. Evidence of pair bonds influencing same-sex bonds is now reported for female olive baboons (Silk et al., 2017) where pair bonds are crucial in parental care and, during certain female reproductive stages, future male mating success (Städele et al., 2019). Male friends shared between females cause female-female bonds to be less biased toward kin and peers than in other savannah baboons (Silk et al., 2017). Stable pair bonds in chimpanzees are also linked to future paternity, suggesting male mating effort can be crucial to the emergence of pair bonds (Langergraber et al., 2013).

Stable male-female social and breeding bonds in Guinea baboons are of high value for primary males as they confer direct fitness benefits, resulting in 98.6\% of copulations (Goffe et al., 2016) and 89.5\% of paternities (chapter 4). As reproduction is non-seasonal (Boese, 1973) and male interactions with females are independent of reproductive state (Goffe et al., 2016), investment in heterosexual bonds must be continuous and year-round. While the dynamics of female transfers are unclear due to rare direct observations, transfers do occur across all social levels, usually involve individual females, and are unambiguous, with females showing immediate preferences for new male partners (personal observation; Goffe et al., 2016). The period following a transfer is usually characterized by high rates of mutual affiliation and close proximity between newly established partners (Goffe, 2016), likely necessary in establishing the new bond. As male time budget is limited by other activities such as feeding or traveling (Dunbar, 1992), social investment in bonds with females is restricted by the time devoted to other social partners (Dunbar, 2004; Lehmann et al., 2007) and investment in each female may also be affected by the total number of associated females. Several reports indicate that a female transferring to a new unit can be followed shortly by one of the resident females of this new unit transferring away to another unit (personal observation; Goffe, 2016). High social investment in newly acquired females could mean decreased investment in relationships with already bonded females either directly causing them to leave or, indirectly, by allowing them to socialize with other males due to decreased vigilance. In a few cases a female transfer occurred directly after a short period of repeated aggression between a female and her primary male (personal observation), indicative of decreased bond quality. While systematic long-term investigations of the influence of pair bond quality and/or mate choice on female transfers are needed, this is not unprecedented. In experimental studies on hamadryas baboons, female social preference for the leader male lead to fewer takeover attempts by other males (Bachmann and Kummer, 1980). 
The fact that inter-sexual dynamics are not simply established by coercion in Guinea baboons further strengthens the importance of pair bond quality. Rates of aggression towards females are less than half of those in geladas and hamadryas baboons (Goffe et al., 2016) and repeated herding behavior and "conditioning" of newly acquired females (as reported in hamadryas baboons Swedell and Schreier, 2009) does not occur (Goffe, 2016). On the contrary, pair bonds are characterized by high levels of tolerance (Goffe, 2016; Fischer et al., 2017). Males maintain proximity to their females, but females interact with secondary males, have high levels of spatial freedom, and respond to male aggression with occasional counter-aggression and female-female coalitions (Goffe, 2016; Goffe et al., 2016; Fischer et al., 2017). Thus, in this fluid and tolerant society where females play an active role in intersexual relationships, stable and strong pair bonds may require higher levels of male investment in the form of affiliation and vigilance, which impose increased limitations on time budget.

\subsection{Male-male relationship dynamics and potential benefits}

Male investment in bonds with males depend on time devoted to other influential activities such as sexual consortship or stable bonds with females (Weingrill et al., 2003; Ostner et al., 2013; GirardButtoz et al., 2014b; Schülke et al., 2014; Goffe et al., 2016; Haunhorst et al., 2016), offspring care and protection (Buchan et al., 2003; Arseneau et al., 2015; Baniel et al., 2016; Minge et al., 2016; Rosenbaum et al., 2018; Städele et al., 2019), and foraging and resting (Dunbar, 1992; Dunbar et al., 2009; Bettridge et al., 2010; Korstjens et al., 2010; Kulahci et al., 2015). Accordingly, while partner preference is stable over time in Guinea baboons, time invested in socializing with other males is negatively affected by number of associated females (chapter 4). Similar effects are seen in male Barbary macaques that also form strong bonds (Young et al., 2014b) with investment in male-male affiliative relationships dropping during the mating season (Berghänel et al., 2011b, 2011a; Young et al., 2014b). My findings show this effect is not restricted to seasonally breeding species and raises new questions regarding if/how these relationships are maintained over time. Several reports of males engaging in frequent affiliation with males close to their unit following the loss of their primary status indicate these relationships could be maintained over time (unpublished data).

As grooming is time consuming (Dunbar, 2004; Lehmann et al., 2007), different behavioral strategies to maintain group cohesion and bonds may be necessary (Dunbar, 2004; Lehmann et al., 2007; Arlet et al., 2015; Kulahci et al., 2015; Kalbitz et al., 2017). In Japanese macaques and ringtail lemurs, vocal exchanges complement grooming and function as an "at distance" bonding mechanism to overcome time constraints and spatial separation (Arlet et al., 2015; Lemur catta: Kulahci et al., 2015). Ritualized triadic male-infant interactions are proposed to be most efficient in maintaining bonds in Assamese macaques due to their quick and mutual nature (Kalbitz et al., 2017). Guinea baboon males similarly

exchange ritualized greeting interactions (chapter 3) that involve potentially costly exchanges (Whitham and Maestripieri, 2003) such as mutual manipulation of the genitalia (chapter 3). It is ar- 
gued that these ritualized exchanges constitute honest affiliative signals due to their high potential costs and are used to test relationship strength (Whitham and Maestripieri, 2003; p. 856). I similarly find that the most intense forms of male-male ritualized greetings are more likely to occur between dyads with higher affiliation rates (chapter 3). Due to their brief and stylized nature, greetings may be an ideal behavioral strategy for Guinea baboon males to assess and maintain relationships after acquiring their own unit. Examining how the dynamics of male-male greetings and other behavioral exchanges play out over time and in relation to social status will be crucial to reveal whether relationships persist throughout male "reproductive careers".

Reproductive success in Guinea baboons most likely depends on the number of associated females and the length of each of these associations, as well as on how early in life males can acquire a unit and how long they maintain primary male status (also see Alberts, 2012 for a review on primates). In our population, secondary males are typically either adolescents or older males (chapter 4, also see Goffe et al. 2016). Virtually all males in their prime have their own unit and it is very rare to observe prime males without associated females (personal observation, chapter 4). Once primary status is established, males tend to maintain this status until post-prime/old age, when they start losing their females one by one (personal observation, chapter 4). The fact that pair bonds require stable yearround investments independent of reproductive state (Goffe et al., 2016) suggests there may be an upper limit to the number of females a male can successfully associate with (see section 5.5). Thus, establishing units as early as possible and extending the reproductive lifespan as long as possible may be the main factors influencing male reproductive success. While male-male sociality does not increase the number of associated females in the short-term, it may be that strong bonds are beneficial in the form of early unit establishment, delayed loss of primary status, or number of females over the entire reproductive lifespan. Guinea baboon males could also adopt different long-term reproductive strategies, as described in horses (Feh, 1999), that are, in turn, affected by factors such as female choice and sex ratio (i.e. female availability). Successful primary males may opt for a "solo strategy" by not heavily investing in strong bonds with other males and focusing their social effort almost exclusively on females. Alternatively, primary males that are not successful with a solo strategy may be better off maintaining relationships with other males (i.e. "social strategy") while balancing time invested in females. This alternative strategy may make the best of a bad situation or even confer different benefits in the form of tolerance and support from strongly bonded males and result in longer breeding tenure. Male strategies can also vary during life history with investment in strong bonds and cooperation being most crucial at specific ages. In Barbary macaques the frequency of coalitionary support is age-dependent, with males relying more heavily on cooperative strategies during their postprime phase (Rathke et al., 2017). The fact that female unit size affects male bonds (chapter 4) makes this a possibility in Guinea baboons as well. If male strategies depend on age and stage of the repro- 
ductive career, this could explain the lack of a link between male bonds and short-term reproductive success in my results and require separate examinations at each of the different life stages.

\subsection{An evolutionary perspective}

Several theoretical frameworks have been developed to explain the great diversity of social systems in the genus Papio and the emergence of multilevel societies in hamadryas and Guinea baboons (Grueter et al., 2012). According to ecological models, nested societies evolved from ancestral multi-male multi-female groups in response to harsh environmental conditions, such as semi-desert habitat in the case of hamadryas baboons, where sparse and scarce food resulted in small female groups that could be monopolized by a single male (Kummer, 1968; Henzi and Barrett, 2003, 2005; Swedell and Plummer, 2012; reviewed in Grueter et al., 2012). Stable one-male units, characterized by male herding behavior, then emerged to benefit from the advantages of permanent consortships (Bergman, 2006), which include high paternity certainty and reduced infanticide (Henzi and Barrett, 2003, 2005; reviewed in Grueter et al., 2012). While fission would be key during foraging, several units aggregating regularly near sleeping sites and water resources, or due to predation pressure, led to the formation of higher, nested social levels (Grueter et al., 2012). While intriguing, models based only on ecological factors fail to explain the emergence of Guinea baboon multilevel societies in a wide range of diverse habitats (Jolly, unpublished manuscript; Galat-Luong et al., 2006). Moreover, multilevel social systems did not emerge in chacma or olive baboons even though several chacma baboon populations live in semi-desert habitats and olive baboon populations occur in habitats similar to Guinea baboons (Jolly, unpublished manuscript; Grueter et al., 2012).

An alternative was proposed by Jolly (2007, 2009; unpublished manuscript) in his "frontier hypothesis", which argues that hamadryas and Guinea baboon societies emerged primarily as a result of demographic factors. A rapid northward expansion into uncontested baboon-free territory resulted in high population growth and favored male philopatry in frontier areas where few and distant neighboring groups resulted in higher migration costs (Jolly, 2009; Jolly, unpublished manuscript). Male philopatry would be additionally enforced via joint defense with related males against potential immigrants (Jolly, 2009). The occupation of baboon-free territories enabled the emergence of larger groups, which resulted in lower inbreeding risks (Jolly, 2009). Groups would split to forage in periods of poor ecological conditions, but subgroups would interact regularly at sleeping sites and water places to maintain critical group breeding size (Jolly, 2009). This led to the multilevel social systems of hamadryas and Guinea baboons (Swedell and Plummer, 2012; Patzelt et al., 2014). In these large groups male philopatry facilitates the emergence of cooperation and tolerance between related/familiar males (chapter 4; Städele et al., 2016; Fischer et al., 2017), while the multilevel social structure reduces the costs of female dispersal due to familiarity between different levels of the society (Kopp, 2015; Städele et al., 2015; Fischer et al., 2017). It is within these larger groups that hamadryas and 
Guinea baboon pair bonds evolved (Swedell and Plummer, 2012; Chowdhury et al., 2015; Goffe et al., 2016; also see chapter 4). Henzi and Barrett (2003, 2005) hypothesized that these associations originated from intense and continuous male mate guarding of small female foraging groups. This led to high paternity certainty, which forced males to stay close to prevent infanticide. Bergman (2006) also found evidence for these pair bonds emerging as a result of permanent consortships and argued that frequent subgrouping led males to develop following and herding strategies outside the fertile phase. Grueter and colleagues (2012) hypothesized that male-female bonds emerged as a response to life in large groups with many unfamiliar males, which led females to opt for opposite-sex associations for protection from harassment and infanticide. While these scenarios start from different assumptions, they lead to similar outcomes, with stable pair bonds benefitting both females and males (Bergman, 2006).

According to Jolly, both hamadryas and Guinea baboons are thought to have evolved from populations at the frontier (Jolly, 2009). Guinea baboons are likely more similar to the ancestral frontier populations, while hamadryas baboons are adapted to the harsher semi-desert habitats of the Horn of Africa (Jolly, unpublished manuscript). In these conditions subgroups would be smaller compared to those in less harsh habitats, such as Guinea baboons, resulting in spatially segregated polygynous reproductive units characterized by male herding behavior (see above; Jolly, unpublished manuscript). At the time Jolly proposed his theory, data on individually identified wild Guinea baboons were not available. Now the evidence from our population supports Jolly's model (2007, 2009; unpublished manuscript). Similar to hamadryas baboons (Swedell and Plummer, 2012; Städele et al., 2015), Guinea baboons live in multilevel societies characterized by female-biased dispersal and stable units (Patzelt et al., 2014; Kopp et al., 2015; Goffe et al., 2016; Fischer et al., 2017). In both species female-female associations are primarily affected by unit membership (Schreier and Swedell, 2009; Goffe et al., 2016), while association and bonding between related/familiar males constitute the core of these societies (chapter 4; Städele et al., 2016; also see discussion in Fischer et al. 2017). Moreover, both species lack male agonistically-enforced rank, have lower male-male aggression rates, as well as greater tolerance compared to other members of this genus (chapters 3 and 4; Kummer, 1997; Swedell and Plummer, 2012; Patzelt et al., 2014; Kalbitzer et al., 2015; Fischer et al., 2017), possibly due to the high potential costs of fighting (Henzi and Barrett, 2003).

Results from our population additionally support Jolly's idea that differing social strategies likely reflect different ecological conditions (Jolly, unpublished manuscript). Likely due to adaptation to more arid habitats, contemporary populations of hamadryas baboons are strictly modular with high fissionfusion dynamics, while contemporary populations of Guinea baboons show weaker fission-fusion dynamics (Grueter et al., 2012) and a fluid social system (chapter 4, sections 5.2 and 5.3, also see Grueter et al. 2012). Although comparative behavioral studies such as between chacma and Guinea baboons (Kalbitzer, 2014; Kalbitzer et al., 2015) are lacking between hamadryas and Guinea baboons, 
findings suggest differences in spatial and behavioral patterns. Combined with my findings, Guinea baboons seem to present higher levels of spatial tolerance and affiliation than hamadryas baboons (chapters 3 and 4, section 5.3). Recent comparisons of African and Asian elephants find that higher availability of resources in more mesic habitats, combined with increased social fluidity in the latter species, led to less despotic and hierarchical social dynamics (Loxodonta africana and Elephas maximus: de Silva et al., 2017). Similar factors, combined with differentiated cooperation and strong bonds, may have facilitated the emergence of the relaxed and tolerant system of Guinea baboons. The differing features and functions of ritualized behaviors in the two species (see section 5.2) also support this conclusion. Ritualized greetings in hamadryas are less elaborate and intense and are usually associated with male status (Colmenares, 1990, 1991b). These interactions tend to occur in aggressive and competitive contexts and function as a tension buffering mechanism (Colmenares, 1991b; Colmenares et al., 2000). Guinea baboon greetings are more elaborate, intense and reciprocated (chapter 3). Most importantly, these interactions are not linked to tension or competition, but function to signal party membership and assess relationships quality (chapter 3). If Guinea baboons evolved from similar frontier populations (Jolly, 2009; Jolly, unpublished manuscript) characterized by large multilevel societies, greetings would have been key in regulating relationships between the numerous males in this fluid system. As I show for a contemporary population (chapter 3), greetings indeed function to delineate social levels and party membership. This parallels human intense and extreme rituals, which are used to signal membership and commitment or in-group values and are linked to the emergence of cooperation and prosociality (Sosis and Bressler, 2003; Boyd, 2006; Henrich, 2009; Xygalatas et al., 2013). Similarly, the most intense and mutual ritualized greeting behaviors are associated with higher levels of male-male tolerance and cooperation in the genus Papio (chapter 3; also see Henzi et al., 2008; De Marco et al., 2014; De Marco, 2017a). At this stage, however, it is not clear whether intense and ritualized interactions and higher levels of spatial tolerance and cooperation emerged in response to similar evolutionary forces, or one feature promoted the emergence of the other in Guinea baboons.

Stable pair bonds also evolved in the mesic and rich habitat of Guinea baboons (Goffe et al., 2016; Fischer et al., 2017), but with different features than hamadryas baboons (Swedell and Saunders, 2006; Swedell and Schreier, 2009). One of the most striking differences is the fact that infanticide episodes were not observed and therefore seem to be extremely rare or absent (Goffe et al., 2016). In Guinea baboon societies, where males are predominantly philopatric and male immigration is rare (Kopp et al., 2015), the threat of infanticide from immigrant males from outer levels of the society is virtually absent. Furthermore, rare "would-be" immigrants are outnumbered by long-term resident males who could all be the potential fathers of these offspring. As the mesic and less arid environment of Guinea baboons led to less frequent subgrouping, monopolization of female groups (resulting in more certain paternities) would have been less straightforward compared to hamadryas ba- 
boons, which present frequent subgrouping, intense male coercion, and lower levels of spatial tolerance between units. In conjunction with the higher levels of spatial tolerance and the more fluid social system (allowing more female freedom, discussed in Fischer et al. 2017) this may have resulted in less certain paternities compared to other baboon species. Combined with male philopatry infanticide would not have constituted a beneficial strategy and could have had detrimental effects on indirect fitness benefits.

Another striking difference of Guinea baboon pair bonds is the lack of intense male coercion and herding behavior typical of hamadryas baboons (Swedell and Schreier, 2009). Relatedness between male competitors is argued to relax competition and decrease levels of harassment and aggression towards females, which otherwise have detrimental effects on female reproductive success and male indirect fitness benefits (Rankin, 2011; Wild et al., 2011; Pizzari and Gardner, 2012; Pitnick and Pfennig, 2014). Although there is a lack of research on the fitness consequences for females, recent research on fruit flies found that relatedness reduced male-male competition and led to less aggressive courtship behavior and slower female reproductive senescence (Drosophila melanogaster: Carazo et al., 2014; also see Pitnick and Pfennig, 2014). Even more interesting, a study trying to replicate these findings, found that relatedness alone is insufficient to explain the reduced harassment towards females and, instead, results from higher familiarity between males reared in the same environment (Hollis et al., 2015). This could hint at male-male familiarity in more fluid societies facilitating the development of lower levels of competition and leading to a lack of intense herding behavior in Guinea baboons. Sexual coercion may constitute a good reproductive strategy for hamadryas baboons, but for Guinea baboons that inhabit less extreme conditions, intense male coercion and herding is too costly for both females (Beehner et al., 2005; Engh et al., 2006b; Sundaresan et al., 2007; Wittig et al., 2008; Swedell and Schreier, 2009; Swedell et al., 2014; Baniel et al., 2017; Zipple et al., 2017) and males (Alberts et al., 1996; Girard-Buttoz et al., 2014b, 2014a; Lukas et al., 2014). The lack of intense female coercion and herding behavior combined with the tolerant social style (Fischer et al., 2017), likely resulted in the higher levels of spatial freedom and female leverage in intersexual relationships (Smuts and Smuts, 1993) observed in Guinea baboons (Goffe et al., 2016). While my thesis confirms the role for pair bonds in male Guinea baboons due to the high proportion of sired offspring within-units (chapter 4), detailed information regarding female choice and male-female association dynamics (including transfers) are still unclear. Moreover, the potential influence of female quality and male mate choice in these dynamic remains unexplored. My findings, nevertheless, suggest high relationship quality may play a crucial role in these pair bonds (section 5.5). 


\section{Conclusions and Outlook}

By using data on social behavior, genetic relatedness and paternity from adolescent and adult males, my thesis provides the first systematic investigation of the adaptive benefits of male-male sociality and of the function of male-male ritualized greeting behavior in wild Guinea baboons. Ritualized greeting behavior plays a key role in regulating male social dynamics, signaling commitment within parties, testing relationships with spatially tolerant partners, and accentuating relationship strength between highly affiliated males. I describe how greetings balance the costs of male co-residence while maintaining group cohesion in this fluid multilevel society. I contribute to current debates regarding associations between ritualized behaviors and social systems by showing that Guinea baboon greetings occur independent of competitive context and are more intense and mutual than in less cooperative and tolerance species. This reinforces the theory that rituals developed in parallel with higher levels of cooperation and reduced aggression in both non-human primates and humans.

I contribute to existing evidence that male-male bonds form in species with stable male co-residence and low contest potential and are linked to greater levels of cooperation. I also show that, while kinship facilitated the evolution of bonds, it is likely not a prerequisite. This corroborates recent assertions that mutualistic processes are a more likely mechanism in the evolution of social bonds. However, while male bonds and cooperation result in substantial fitness benefits in many mammalian species, I show no positive link between male-male sociality and short-term reproductive success. Instead, this first investigation into male Guinea baboon paternity shows the value of pair bonds in securing high paternity certainty. The importance of pair bonds and relatively high female leverage result in males shifting investment towards intersexual relationships resulting in a decrease in time spent socializing with other males. My thesis provides an important contribution to the field and our understanding of the Guinea baboon social system. My findings also highlights unresolved questions and new avenues for investigation.

Understanding male-male relationship dynamics over life history and in relation to reproductive status is necessary to assess if/how bonds are maintained over time. I discussed (section 5.6) how intense greetings could be crucial to relationship maintenance. It would be important to determine whether changes over time in directionality and symmetry of grooming and approaches are affected by male reproductive status of either partner. While I show investment in male-male affiliation is influenced by changes in associations with females, whether this pattern holds for coalitionary support is not yet known. It would also be key to test whether primary males are differentially interested in the affiliative or scream vocalizations of friends versus non-friends. Playback experiments could be used to test reactions to vocalizations of "ex-friends" (i.e. top partners during adolescence) versus "nonex-friends" (i.e. less connected partners during adolescence). 
My results also open new questions regarding female choice and pair bond dynamics. In particular, what female Guinea baboons are attracted to and what factors affect transfers. Possible avenues of investigation are to test whether females favor males that provide more conflict support, affiliation, tolerance, or infant/yearling care. Investigating the effect of potential signals of status, such as mane size and hind-quarter coloration, have on female association and reproductive success could help determine if these advertise male condition, which would highlight a shift from fighting-related physical features. Lastly, it is fundamental to investigate if pair bond quality plays a role in these associations by testing if male investment in affiliation and/or vigilance have an effect on female transfers. To achieve this, long-term data could be used to investigate social dynamics in both pre- and post-transfer units and variations in the frequency and equitability of grooming and approaches with the primary and other males. Analyses should also include variations in relative frequencies of affiliative and hostile behavior (tenor), comparing pair bonds at 0 month, 3 months and 6 months before transfers.

While I investigated the short-term reproductive benefits of sociality in male Guinea baboons, the potential long-term benefits remain unclear. Male sociality may result in enhanced fitness in the form of females, offspring and tenure length over the entire reproductive lifespan. Moreover, using crosssectional data from the start and end of male reproductive careers could uncover if more social males gain benefits through early unit establishment or delayed unit loss. It may be that relationships combat the detrimental effects of isolation regardless of the sex of the social partner. Indeed, an unexplored area of Guinea baboon research is any link between sociality and physiological responses to social and environmental stressors. One could test whether "social isolation", measured as lower interaction rates with females, infants, and other males, affects glucocorticoid levels. The physiological and social responses to the loss of a close social partner could also highlight the role of social ties in this species and how individuals cope with drastic changes in social dynamics. Lastly, environmental stressors, such as the harsh dry seasons and droughts observed in recent years, may serve to examine the effects of climate change and whether individuals with higher levels of sociality and support are more resilient to such changes. 


\section{References}

Abegglen, J.-J., 1984. On socialization in hamadryas baboons: a field study. Bucknell University Press, London.

Alberts, S.C., 2012. Male reproductive performance. In: Mitani, J.C., Call, J., Kappeler, P.M., Palombit, R.A., Silk, J.B. (Eds.), The Evolution of Primate Societies. University of Chicago Press, London Chicago, pp. 412-431.

Alberts, S.C., Altmann, J., Wilson, M.L., 1996. Mate guarding constrains foraging activity of male baboons. Animal Behaviour. 51, 1269-1277.

Alberts, S.C., Buchan, J.C., Altmann, J., 2006. Sexual selection in wild baboons: from mating opportunities to paternity success. Animal Behaviour. 72, 1177-1196.

Alberts, S.C., Watts, H.E., Altmann, J., 2003. Queuing and queue-jumping: long-term patterns of reproductive skew in male savannah baboons, Papio cynocephalus. Animal Behaviour. 65, 821-840.

Alexander, R.D., 1974. The evolution of social systems. Annual review of ecology and systematics. 5(1), 325-383.

Altizer, S., Nunn, C.L., Thrall, P.H., Gittleman, J.L., Antonovics, J., Cunningham, A.A., Dobson, A.P., Ezenwa, V., Jones, K.E., Pedersen, A.B., Poss, M., Pulliam, J.R.C., 2003. Social Organization and Parasite Risk in Mammals: Integrating Theory and Empirical Studies. Annual Review of Ecology, Evolution, and Systematics. 34, 517-547.

Altmann, J., 1974. Observational study of behavior: sampling methods. Behaviour. 49, 227-266.

Altmann, J., 1990. Primate males go where the females are. Animal Behaviour. 39, 193-195.

Altmann, S.A., 1962. A field study of the sociobiology of rhesus monkeys. Annals of the New York Academy of Sciences. 102, 338-435.

Andrews, R. V., Belknap, R.W., 1986. Bioenergetic benefits of huddling by deer mice (Peromyscus maniculatus). Comparative biochemistry and physiology - Part A - Comparative physiology. 85(4), 775-778.

Aplin, L.M., Farine, D.R., Mann, R.P., Sheldon, B.C., 2014. Individual-level personality influences social foraging and collective behaviour in wild birds. Proceedings of the Royal Society B: Biological Sciences. 281, 20141016.

Archie, E.A., Tung, J., Clark, M., Altmann, J., Alberts, S.C., Archie, E.A., 2014. Social affiliation matters: both same-sex and opposite-sex relationships predict survival in wild female baboons. Proceedings of the Royal Society B: Biological Sciences. 281, 20141261.

Arlet, M., Jubin, R., Masataka, N., Lemasson, A., 2015. Grooming-at-a-distance by exchanging calls in non-human primates. Biology Letters. 11, 20150711.

Arseneau-Robar, T.J.M., Müller, E., Taucher, A.L., van Schaik, C.P., Willems, E.P., 2016. Male food defence as a by-product of intersexual cooperation in a non-human primate. Scientific Reports. 6 , 35800 .

Arseneau, T.J.M., Taucher, A., van Schaik, C.P., Willems, E.P., 2015. Male monkeys fight in betweengroup conflicts as protective parents and reluctant recruits. Animal Behaviour. 110, 39-50.

Aureli, F., Schaffner, C.M., 2007. Aggression and conflict management at fusion in spider monkeys. Biology letters. 3, 147-149.

Baayen, R.H., 2008. Analyzing linguistic data: A practical introduction to statistics using R. Cambridge University Press, Cambridge. 
Bachmann, C., Kummer, H., 1980. Male assessment of female choice in hamadryas baboons. Behavioral Ecology and Sociobiology. 6, 315-321.

Baldellou, M., Henzi, P., 1992. Vigilance, predator detection and the presence of supernumerary males in vervet monkey troops. Animal Behaviour. 43, 451-461.

Baniel, A., Cowlishaw, G., Huchard, E., 2016. Stability and strength of male-female associations in a polygynous primate society. Behavioral Ecology and Sociobiology. 70, 761-775.

Baniel, A., Cowlishaw, G., Huchard, E., 2017. Male violence and sexual intimidation in a wild primate society. Current Biology. 27, 2163-2168.

Barr, D.J., Levy, R., Scheepers, C., Tily, H.J., 2013. Random effects structure for confirmatory hypothesis testing: Keep it maximal. Journal of Memory and Language. 68, 255-278.

Bates, D., Mächler, M., Bolker, B., Walker, S., 2015. Fitting Linear Mixed-Effects Models using lme4. Journal of Statistical Software. 67, 1-48.

Beehner, J.C., Bergman, T.J., 2008. Infant mortality following male takeovers in wild geladas. American Journal of Primatology. 70, 1152-1159.

Beehner, J.C., Bergman, T.J., Cheney, D.L., Seyfarth, R.M., Whitten, P.L., 2005. The effect of new alpha males on female stress in free-ranging baboons. Animal Behaviour. 69, 1211-1221.

Benenson, J.F., Markowitz, H., Emery Thompson, M., Wrangham, R.W., 2009. Strength determines coalitional strategies in humans. Proceedings of the Royal Society B: Biological Sciences. 276, 2589-2595.

Berard, J.D., Nurnberg, P., Epplen, J.T., Schmidtke, J., 1994. Alternative reproductive tactics and reproductive success in male rhesus macaques. Behaviour. 129, 177-201.

Bercovitch, F.B., 1988. Coalitions, cooperation and reproductive tactics among adult male baboons. Animal Behaviour. 36, 1198-1209.

Bercovitch, F.B., Widdig, A., Trefilov, A., Kessler, M.J., Berard, J.D., Schmidtke, J., Nürnberg, P., Krawczak, M., 2003. A longitudinal study of age-specific reproductive output and body condition among male rhesus macaques, Macaca mulatta. Naturwissenschaften. 90, 309-312.

Berghänel, A., Ostner, J., Schröder, U., Schülke, O., 2011a. Social bonds predict future cooperation in male Barbary macaques, Macaca sylvanus. Animal Behaviour. 81, 1109-1116.

Berghänel, A., Ostner, J., Schülke, O., 2011b. Coalitions destabilize dyadic dominance relationships in male Barbary macaques (Macaca sylvanus). Behaviour. 148, 1256-1274.

Bergman, T.J., 2006. Hybrid baboons and the origins of the hamadryas male reproductive strategy. In: Swedell, L., Leigh, S.R. (Eds.), Reproduction and fitness in baboons: Behavioral, ecological, and life history perspectives. Springer, New York, pp. 81-103.

Bergman, T.J., 2010. Experimental evidence for limited vocal recognition in a wild primate: Implications for the social complexity hypothesis. Proceedings of the Royal Society B: Biological Sciences. 277, 3045-3053.

Bergman, T.J., Ho, L., Beehner, J.C., 2009. Chest color and social status in male geladas (Theropithecus gelada). International Journal of Primatology. 30, 791-806.

Best, E.C., Dwyer, R.G., Seddon, J.M., Goldizen, A.W., 2014. Associations are more strongly correlated with space use than kinship in female eastern grey kangaroos. Animal Behaviour. 89, 1-10.

Bettridge, C., Lehmann, J., Dunbar, R.I.M., 2010. Trade-offs between time, predation risk and life history, and their implications for biogeography: A systems modelling approach with a primate case study. Ecological Modelling. 221, 777-790.

Bissonnette, A., Bischofberger, N., van Schaik, C.P., 2011. Mating skew in Barbary macaque males: The role of female mating synchrony, female behavior, and male-male coalitions. Behavioral Ecology and Sociobiology. 65, 167-182. 
Bissonnette, A., de Vries, H., van Schaik, C.P., 2009. Coalitions in male Barbary macaques, Macaca sylvanus: strength, success and rules of thumb. Animal Behaviour. 78, 329-335.

Bissonnette, A., Franz, M., Schülke, O., Ostner, J., 2014. Socioecology, but not cognition, predicts male coalitions across primates. Behavioral Ecology. 25, 794-801.

Blouin, M.S., 2003. DNA-based methods for pedigree reconstruction and kinship analysis in natural populations. Trends in Ecology and Evolution. 18, 503-511.

Blumstein, D.T., Williams, D.M., Lim, A.N., Kroeger, S., Martin, J.G.A., 2018. Strong social relationships are associated with decreased longevity in a facultatively social mammal. Proceedings of the Royal Society B: Biological Sciences. 285, 20171934.

Boesch, C., 1994. Cooperative hunting in wild chimpanzees. Animal Behaviour. 48, 653-667.

Boesch, C., Boesch, H., Vigilant, L., 2006a. Cooperative hunting in chimpanzees: kinship or mutualism? In: Kappeler, P.M., van Schaik, C.P. (Eds.), Cooperation in Primates and Humans. Springer, Berlin, Heidelberg, pp. 139-150.

Boesch, C., Kohou, G., Néné, H., Vigilant, L., 2006b. Male Competition and Paternity in Wild Chimpanzees of the Taï. American Journal of Physical Anthropology. 130, 103-115.

Boese, G.K., 1973. Behavior and Social Organization of the Guinea Baboon (Papio papio). Doctoral dissertation, Johns Hopkins University, USA.

Boese, G.K., 1975. Social behavior and ecological considerations of West African baboons (Papio papio). In: Russell H. Tuttle, E. (Ed.), Socioecology and Psychology of Primates. The Hague, Chicago, pp. 205-230.

Boinski, S., 1994. Affiliation patterns among male Costa rican squirrel monkeys. Behaviour. 130, 191209.

Bowerman, B.L., O'connell, R.T., 1990. Linear statistical models: An applied approach, 2nd ed. Duxbury Press, Pacific Grove CA.

Boyd, R., 2006. The Puzzle of Human Sociality. Science. 314, 1555-1556.

Braun, A., Bugnyar, T., 2012. Social bonds and rank acquisition in raven nonbreeder aggregations. Animal Behaviour. 84, 1507-1515.

Bray, J., Pusey, A.E., Gilby, I.C., 2016. Incomplete control and concessions explain mating skew in male chimpanzees. Proceedings of the Royal Society B: Biological Sciences. 283, 20162071.

Brent, L.J.N., Heilbronner, S.R., Horvath, J.E., Gonzalez-Martinez, J., Ruiz-Lambides, A., Robinson, A.G., Skene, J.H.P., Platt, M.L., 2013. Genetic origins of social networks in rhesus macaques. Scientific Reports. 3, 1042.

Buchan, J.C., Alberts, S.C., Silk, J.B., Altmann, J., 2003. True paternal care in a multi-male primate society. Nature. 425, 179-181.

Bulger, J.B., 1993. Dominance rank and access to estrous females in male savanna baboons. Behaviour. 127, 67-103

Cameron, E.Z., Setsaas, T.H., Linklater, W.L., 2009. Social bonds between unrelated females increase reproductive success in feral horses. Proceedings of the National Academy of Sciences. 106, 13850-13853.

Carazo, P., Tan, C.K.W., Allen, F., Wigby, S., Pizzari, T., 2014. Within-group male relatedness reduces harm to females in Drosophila. Nature. 505, 672.

Carnes, L.M., Nunn, C.L., Lewis, R.J., 2011. Effects of the Distribution of Female Primates on the Number of Males. PLoS ONE. 6, e19853.

Carter, G.G., Farine, D.R., Wilkinson, G.S., 2017. Social bet-hedging in vampire bats. Biology letters. 13, 20170112. 
Carter, G.G., Wilkinson, G.S., 2015. Social benefits of non-kin food sharing by female vampire bats. Proceedings of the Royal Society B: Biological Sciences. 282, 20152524.

Cheney, D.L., Silk, J.B., Seyfarth, R.M., 2016. Network connections, dyadic bonds and fitness in wild female baboons. Royal Society Open Science. 3(7), 160255.

Chowdhury, S., Pines, M., Saunders, J., Swedell, L., 2015. The adaptive value of secondary males in the polygynous multi-level society of hamadryas baboons. American Journal of Physical Anthropology. 158, 501-513.

Ciani, F., Dall'Olio, S., Stanyon, R., Palagi, E., 2012. Social tolerance and adult play in macaque societies: A comparison with different human cultures. Animal Behaviour. 84, 1313-1322.

Clutton-brock, T., 2009. Cooperation between non-kin in animal societies. Nature. 461, 51-57.

Clutton-Brock, T., 2002. Breeding together: kin selection and mutualism in cooperative vertebrates. Science. 296, 69-72.

Clutton-Brock, T.H., 1998. Reproductive Skew, Concessions and Limited Control. Science. 13, $288-$ 292.

Clutton-Brock, T.H., 2016. Mammal Societies. John Wiley \& Sons.

Clutton-Brock, T.H., Isvaran, K., 2006. Paternity loss in contrasting mammalian societies. Biology Letters. 2, 513-516.

Colmenares, F., 1990. Greeting Behaviour in Male Baboons, I: Communication, Reciprocity and Symmetry. Behaviour. 113, 81-115.

Colmenares, F., 1991a. Greeting, aggression, and coalitions between male baboons: Demographic correlates. Primates. 32, 453-463.

Colmenares, F., 1991b. Greeting behaviour between male baboons: oestrous females, rivalry and negotiation. Animal Behaviour. 41, 49-60.

Colmenares, F., 1992. Clans and harems in a colony of hamadryas and hybrid baboons: male kinship, familiarity and the formation of brother-teams. Behaviour. 121, 61-93.

Colmenares, F., Esteban, M.M., Zaragoza, F., 2007. One-male units and clans in a colony of hamadryas baboons (Papio hamadryas hamadryas): Effect of male number and clan cohesion on feeding success. American Journal of Primatology. 69, 21-37.

Colmenares, F., Hofer, H., East, M.L., 2000. Greeting ceremonies in baboons and hyenas. In: Aureli, F, de Waal, F.B.M. (Eds.), Natural conflict resolution. University of California Press, Berkeley, pp. 94-96.

Connor, R.C., Krützen, M., 2015. Male dolphin alliances in Shark Bay: Changing perspectives in a 30year study. Animal Behaviour. 103, 223-235.

Connor, R.C., Wells, R.S., Mann, J., Read, A.J., 2000. The bottlenose dolphin: social relationships in a fission-fusion society. In: Mann, J., Conner, R.C., Tyack, P.L., Whitehead, H. (Eds.), Cetacean Societies: Field Studies of Dolphins and Whales. Chicago, pp. 91-126.

Cowlishaw, G., Dunbar, R.I.M., 1991. Dominance rank and mating success in male primates. Animal Behaviour. 41, 1045-1056.

Crawley, M.J., 2002. Statistical computing: an introduction to data analysis using S-Plus. Wiley, Chichester.

Creel, S., Creel, N.M., 1995. Communal hunting and pack size in African wild dogs, Lycaon pictus. Animal Behaviour. 50, 1325-1339.

Creel, S.R., Waser, P.M., 1991. Failures of reproductive suppression in dwarf mongooses (Helogale parvula): accident or adaptation? Behavioral Ecology. 2, 7-15. 
Crockford, C., Wittig, R.M., Whitten, P.L., Seyfarth, R.M., Cheney, D.L., 2008. Social stressors and coping mechanisms in wild female baboons (Papio hamadryas ursinus). Hormones and Behavior. $53,254-265$.

Crofoot, M.C., 2007. Mating and feeding competition in white-faced capuchins (Cebus capucinus): the importance of short- and long-term strategies. Behaviour. 144, 1473-1495.

Cullen, J.M., 1966. E. Ritualization of animal activities in relation to phylogeny, speciation and ecology: Reduction of ambiguity through ritualization. Philosophical Transactions of the Royal Society of London. Series B, Biological Sciences. 251, 363-374.

Curley, J.P., 2016. compete: Analyzing competitive interaction data: R package version 0.1. https:// CRAN.R-project.org/package $=$ compete.

Dal Pesco, F., Fischer, J., 2018. Greetings in male Guinea baboons and the function of rituals in complex social groups. Journal of Human Evolution. 125, 87-98.

De Marco, A., 2017. Male greetings. In: The International Encyclopedia of Primatology. John Wiley \& Sons, Inc., Hoboken, NJ, USA.

De Marco, A., Sanna, A., Cozzolino, R., Thierry, B., 2014. The function of greetings in male Tonkean macaques. American Journal of Primatology. 76, 989-998.

de Silva, S., Schmid, V., Wittemyer, G., 2017. Fission-fusion processes weaken dominance networks of female Asian elephants in a productive habitat. Behavioral Ecology. 28, 243-252.

de Waal, F.B.M., 1985. Coalitions in monkeys and apes. In: Wilke, H.A.M. (Ed.), Advances in Psychology. Elsevier, Amsterdam, pp. 1-27.

Dewsbury, D.A., 1982. Dominance rank, copulatory behavior, and differential reproduction. The Quarterly Review of Biology. 57, 135-159.

Dias, P.A.D., Luna, E.R., Espinosa, D.C., 2008. The functions of the "greeting ceremony" among male mantled howlers (Alouatta palliata) on Agaltepec Island, Mexico. American Journal of Primatology. 70, 621-628.

Diaz-Aguirre, F., Parra, G.J., Passadore, C., Möller, L.M., 2018. Kinship influences social bonds among male southern Australian bottlenose dolphins (Tursiops cf. australis). Behavioral Ecology and Sociobiology. 72, 190-203.

Díaz-Muñoz, S.L., DuVal, E.H., Krakauer, A.H., Lacey, E.A., 2014. Cooperating to compete: Altruism, sexual selection and causes of male reproductive cooperation. Animal Behaviour. 88, 67-78.

Douglas, P.H., Hohmann, G., Murtagh, R., Thiessen-Bock, R., Deschner, T., 2016. Mixed messages: wild female bonobos show high variability in the timing of ovulation in relation to sexual swelling patterns. BMC Evolutionary Biology. 16, 140.

Drea, C.M., Carter, A.N., 2009. Cooperative problem solving in a social carnivore. Animal Behaviour. 78, 967-977.

Drews, C., 1993. The concept and definition of dominance in animal behavior. Behaviour. 125, 283313.

Dubuc, C., Hughes, K.D., Cascio, J., Santos, L.R., 2012. Social tolerance in a despotic primate: Cofeeding between consortship partners in rhesus macaques. American Journal of Physical Anthropology. 148, 73-80.

Dubuc, C., Muniz, L., 2011. Testing the priority-of-access model in a seasonally breeding primate species. Behavioral Ecology and Sociobiology. 65, 1615-1627.

Duffy, K.G., Richard, W., Silk, J.B., 2007. Male chimpanzees exchange political support for mating opportunities. Current Biology. 17, 586-587.

Dunbar, R., Dunbar, P., 1975. Social dynamics of gelada baboons. Contributions to primatology. S. Karger AG, Basel, Vol. 6. 
Dunbar, R.I.M., 1983. Relationships and social structure in gelada and hamadryas baboons. In: Hinde, R.A. (Ed.), Primate Social Relationships: An Integrated Approach. Sinauer Associates, Sunderland, MA, pp. 299-307.

Dunbar, R.I.M., 1992. Time: a hidden constraint on the behavioural ecology of baboons. Behavioral Ecology and Sociobiology. 31, 35-49.

Dunbar, R.I.M., 2004. Gossip in evolutionary perspective. Review of General Psychology. 8, 100110.

Dunbar, R.I.M., Korstjens, A.H., Lehmann, J., 2009. Time as an ecological constraint. Biological Reviews. 84, 413-429.

Dunbar, R.I.M., Srivastava, A., 1996. The mating system of Hanuman langurs: a problem in optimal foraging. Behavioral Ecology and Sociobiology. 39, 219-226.

East, M.L., Hofer, H., Wickler, W., 1993. The erect "penis" is a flag of submission in a female-dominated society: greetings in Serengeti spotted hyenas. Behavioral Ecology and Sociobiology. 33, $355-370$.

Ellis, L., 1995. Dominance and reproductive success among nonhuman animals: A cross-species comparison. Ethology and Sociobiology. 16, 257-333.

Ellis, S., Franks, D.W., Nattrass, S., Cant, M.A., Weiss, M.N., Giles, D., Balcomb, K.C., Croft, D.P., 2017. Mortality risk and social network position in resident killer whales: Sex differences and the importance of resource abundance. Proceedings of the Royal Society B: Biological Sciences. 284, 20171313.

Emlen, S., Oring, L., 1977. Ecology, sexual selection, and the evolution of mating systems. Science, 197, 215-223.

Engh, A.L., Beehner, J.C., Bergman, T.J., Whitten, P.L., Hoffmeier, R.R., Seyfarth, R.M., Cheney, D.L., 2006a. Behavioural and hormonal responses to predation in female chacma baboons (Papio hamadryas ursinus). Proceedings of the Royal Society B: Biological Sciences. 273, 707-712.

Engh, A.L., Beehner, J.C., Bergman, T.J., Whitten, P.L., Hoffmeier, R.R., Seyfarth, R.M., Cheney, D.L., 2006b. Female hierarchy instability, male immigration and infanticide increase glucocorticoid levels in female chacma baboons. Animal Behaviour. 71, 1227-1237.

Estes, R.D., Goddard, J., 1967. Prey selection and hunting behavior of the African wild dog. The Journal of Wildlife Management. 31, 52-70.

Farine, D.R., Sánchez-Tójar, A., 2018. aniDom: Inferring dominance hierarchies and estimating uncertainty: R package version 0.1.3. https://CRAN.R-project.org/package=aniDom.

Fashing, P.J., 2001. Male and female strategies during intergroup encounters in guerezas (Colobus guereza): Evidence for resource defense mediated through males and a comparison with other primates. Behavioral Ecology and Sociobiology. 50, 219-230.

Fedigan, L.M., Jack, K.M., Avila, M., Carnegie, S., Gilmore, S., Mackinnon, K., Lamarsh, C., Morris, D., Rose, L., 2004. The demographic and reproductive context of male replacements in Cebus capucinus. Behaviour. 141, 755-775.

Feh, C., 1999. Alliances and reproductive success in Camargue stallions. Animal Behaviour. 57, 705713.

Field, A., Miles, J., Field, Z., 2012. Discovering statistics using R, Choice Reviews Online. Sage Publications, London.

Fischer, J., Kopp, G.H., Dal Pesco, F., Goffe, A., Hammerschmidt, K., Kalbitzer, U., Klapproth, M., Maciej, P., Ndao, I., Patzelt, A., Zinner, D., 2017. Charting the neglected West: The social system of Guinea baboons. American Journal of Physical Anthropology. 162, 15-31.

Fox, J., Weisberg, S., 2011. Mixed-effects models in R. An R Companion to Applied Regression, SAGE: Thousand Oaks, CA, USA. 
Fraser, Ó., Plowman, A.B., 2007. Function of notification in Papio hamadryas. International Journal of Primatology. 28, 1439-1448.

Fraser, O.N., Bugnyar, T., 2010. The quality of social relationships in ravens. Animal Behaviour. 79, 927-933.

Freeland, W.J., 1976. Pathogens and the evolution of primate sociality. Biotropica. 8(1), 12-24.

Frère, C.H., Krützen, M., Mann, J., Connor, R.C., Bejder, L., Sherwin, W.B., 2010. Social and genetic interactions drive fitness variation in a free-living dolphin population. Proceedings of the National Academy of Sciences. 107, 19949-19954.

Furuichi, T., 1989. Social interactions and the life history of femalePan paniscus in Wamba, Zaire. International Journal of Primatology. 10, 173-197.

Galat-Luong A., Galat G., Hagell S., 2006. The social and ecological flexibility of Guinea baboons: Implications for Guinea baboon social organization and male strategies. In: Swedell L., Leigh S.R. (Eds.), Reproduction and fitness in baboons: Behavioral, ecological, and life history perspectives. Developments in Primatology: Progress and Prospects. Springer, Boston, MA, pp. 105-121.

Galbany, J., Tung, J., Altmann, J., Alberts, S.C., 2015. Canine length in wild male baboons: Maturation, aging and social dominance rank. PLoS ONE. 10, 1-16.

Garber, P.A., Kowalewski, M.K., 2011. Collective action and male affiliation in Howler monkeys (Alouatta caraya). In: Sussman, R.W., Cloninger, C.R. (Eds.), Origins of altruism and cooperation. Springer, New York, NY, pp. 145-165.

Georgiev, A. V., Muehlenbein, P.M., Prall, S.P., Emery Thompson, M., Maestripieri, D., 2015. Male quality, dominance rank, and mating success in free-ranging rhesus macaques. Behavioral Ecology. $26,763-772$.

Gilby, I.C., Brent, L.J.N., Wroblewski, E.E., Rudicell, R.S., Hahn, B.H., Goodall, J., Pusey, A.E., 2013. Fitness benefits of coalitionary aggression in male chimpanzees. Behavioral Ecology and Sociobiology. 67, 373-381.

Girard-Buttoz, C., Heistermann, M., Rahmi, E., Agil, M., Fauzan, P.A. hmad, Engelhardt, A., 2014a. Costs of mate-guarding in wild male long-tailed macaques (Macaca fascicularis): physiological stress and aggression. Hormones and behavior. 66, 637-648.

Girard-Buttoz, C., Heistermann, M., Rahmi, E., Marzec, A., Agil, M., Fauzan, P.A., Engelhardt, A., 2014b. Mate-guarding constrains feeding activity but not energetic status of wild male long-tailed macaques (Macaca fascicularis). Behavioral Ecology and Sociobiology. 68, 583-595.

Goffe, A.S., 2016. Social relationships of female Guinea baboons (Papio papio) in Senegal. Doctoral dissertation, Georg-August-Universität Göttingen, Germany.

Goffe, A.S., Fischer, J., 2016. Meat sharing between male and female Guinea baboons (Papio papio). Primate Biology. 3, 1-8.

Goffe, A.S., Zinner, D., Fischer, J., 2016. Sex and friendship in a multilevel society: behavioural patterns and associations between female and male Guinea baboons. Behavioral Ecology and Sociobiology. 70, 323-336.

Garden City, N.Y., Doubleday.

Grinnell, J., Packer, C., Pusey, A.E., 1995. Cooperation in male lions: kinship, reciprocity or mutualism? Animal Behaviour. 49, 95-105.

Gruber, B., Adamack, A.T., 2015. landgenreport: A new R function to simplify landscape genetic analysis using resistance surface layers. Molecular Ecology Resources. 15, 1172-1178.

Grueter, C.C., Chapais, B., Zinner, D., 2012. Evolution of multilevel social systems in nonhuman primates and humans. International Journal of Primatology. 33, 1002-1037.

Hall, K.R.L., DeVore, I., 1965. Baboon social behavior. In: Irven DeVore (Ed.), Primate behavior: Field studies of monkeys and apes. Holt, Rinehart and Winston, New York, pp. 53-110. 
Hamilton, W.D., 1971. Geometry for the selfish herd. Journal of Theoretical Biology. 31, 295-311.

Harris, T.R., 2010. Multiple resource values and fighting ability measures influence intergroup conflict in guerezas (Colobus guereza). Animal Behaviour. 79, 89-98.

Haunhorst, C.B., Heesen, M., Ostner, J., Schülke, O., 2017. Social bonds with males lower the costs of competition for wild female Assamese macaques. Animal Behaviour. 125, 51-60.

Haunhorst, C.B., Schülke, O., Ostner, J., 2016. Opposite-sex social bonding in wild Assamese macaques. American Journal of Primatology. 78, 872-882.

Hausfater, G., Takacs, D., 1987. Structure and function of hindquarter presentations in yellow baboons (Papio cynocephalus). Ethology. 74, 297-319.

Hawkley, L.C., Cole, S.W., Capitanio, J.P., Norman, G.J., Cacioppo, J.T., 2012. Effects of social isolation on glucocorticoid regulation in social mammals. Hormones and Behavior. 62, 314-323.

Heathcote, R.J.P., Darden, S.K., Franks, D.W., Ramnarine, I.W., Croft, D.P., 2017. Fear of predation drives stable and differentiated social relationships in guppies. Scientific Reports. 7, 1-10.

Heesen, M., Rogahn, S., Macdonald, S., Ostner, J., Schülke, O., 2014. Predictors of food-related aggression in wild Assamese macaques and the role of conflict avoidance. Behavioral Ecology and Sociobiology. 68, 1829-1841.

Henrich, J., 2009. The evolution of costly displays, cooperation and religion: Credibility enhancing displays and their implications for cultural evolution. Evolution and Human Behavior. 30, 244 260.

Henzi, P., Barrett, L., 2003. Evolutionary Ecology, Sexual Conflict, and Behavioral Differentiation Among Baboon Populations. Evolutionary Anthropology. 12, 217-230.

Henzi, S.P., Barrett, L., 2005. The historical socioecology of savanna baboons (Papio hamadryas). Journal of Zoology. 265, 215-226.

Henzi, S.P., Clarke, P., Barrett, L., Noë, R., Jolly, C., 2008. The genetics and biogeography of coalition formation in savanna baboons. INCORE Report.

Henzi, S.P., Clarke, P.M.R., van Schaik, C.P., Pradhan, G.R., Barrett, L., 2010. Infanticide and reproductive restraint in a polygynous social mammal. Proceedings of the National Academy of Sciences. 107, 2130-2135.

Henzi, S.P., Hetem, R., Fuller, A., Maloney, S., Young, C., Mitchell, D., Barrett, L., McFarland, R., 2017. Consequences of sex-specific sociability for thermoregulation in male vervet monkeys during winter. Journal of Zoology. 302, 193-200.

Higham, J.P., MacLarnon, A.M., Ross, C., Heistermann, M., Semple, S., 2008. Baboon sexual swellings: Information content of size and color. Hormones and Behavior. 53, 452-462.

Hill, D.A., van Hooff, J.A.R.A.M., 1994. Affiliative relationships between males in groups of nonhuman primates: a summary. Behaviour. 130, 143-149.

Hinde, R.A., 1976. Interactions, Relationships and Social Structure. Man. 11(1), 1-17.

Hirsch, B.T., Prange, S., Hauver, S.A., Gehrt, S.D., 2013. Genetic relatedness does not predict racoon social network structure. Animal Behaviour. 85, 463-470.

Hohmann, G., Fruth, B., 2000. Use and function of genital contacts among female bonobos. Animal behaviour. 60, 107-120.

Hollis, B., Kawecki, T.J., Keller, L., 2015. No evidence that within-group male relatedness reduces harm to females in Drosophila. Ecology and Evolution. 5, 979-983.

Holt-Lunstad, J., Smith, T.B., Baker, M., Harris, T., Stephenson, D., 2015. Loneliness and social isolation as risk factors for mortality. Perspectives on Psychological Science. 10, $227-237$.

Holt-Lunstad, J., Smith, T.B., Layton, J.B., 2010. Social relationships and mortality risk: A meta-analytic review. PLoS Medicine. 7, 1-20. 
Huchard, E., Alvergne, A., Féjan, D., Knapp, L.A., Cowlishaw, G., Raymond, M., 2010. More than friends? Behavioural and genetic aspects of heterosexual associations in wild chacma baboons. Behavioral Ecology and Sociobiology. 64, 769-781.

Hughes, W.O.H., Eilenberg, J., Boomsma, J.J., 2002. Trade-offs in group living: transmission and disease resistance in leaf-cutting ants. Proceedings of the Royal Society of London. Series B: Biological Sciences. 269, 1811-1819.

Jacob, A., Long, A., 2018. Package 'jtools'; version 1.1.1. https://CRAN.R-project.org.

Johnstone, R.A., Cant, M.A., 1999. Reproductive skew and the threat of eviction: a new perspective. Proceedings of the Royal Society of London. Series B: Biological Sciences. 266, 275-279.

Jolly, C.J., 2007. Baboons, mandrills, and mangabeys: Afro-papionin socioecology in a phylogenetic perspective. In: Campbell, C.J. (Ed.), Primates in Perspective. Oxford University Press, pp. 240 251.

Jolly, C.J., 2009. Fifty years of looking at human evolution: Backward, forward, and sideways. Current Anthropology. 50, 187-199.

Jordan, L.A., Wong, M.Y.L., Balshine, S.S., 2010. The effects of familiarity and social hierarchy on group membership decisions in a social fish. Biology Letters. 6, 301-303.

Josephs, N., Bonnell, T., Dostie, M., Barrett, L., Peter Henzi, S., 2016. Working the crowd: Sociable vervets benefit by reducing exposure to risk. Behavioral Ecology. 27, 988-994.

Kalbitz, J., Ostner, J., Schülke, O., 2016. Strong, equitable and long-term social bonds in the dispersing sex in Assamese macaques. Animal Behaviour. 113, 13-22.

Kalbitz, J., Schülke, O., Ostner, J., 2017. Triadic male-infant-male interaction serves in bond maintenance in male Assamese macaques. PLoS ONE. 12, 16-19.

Kalbitzer, U., 2014. Foundations of variation in male aggressiveness and tolerance between chacma baboons (Papio ursinus) in Botswana and Guinea baboons (P. papio) in Senegal. Doctoral dissertation, Georg-August-Universität Göttingen, Germany.

Kalbitzer, U., Bergstrom, M.L., Carnegie, S.D., Wikberg, E.C., Kawamura, S., Campos, F.A., Jack, K.M., Fedigan, L.M., 2017. Female sociality and sexual conflict shape offspring survival in a Neotropical primate. Proceedings of the National Academy of Sciences. 114, 1892-1897.

Kalbitzer, U., Heistermann, M., Cheney, D., Seyfarth, R., Fischer, J., 2015. Social behavior and patterns of testosterone and glucocorticoid levels differ between male chacma and Guinea baboons. Hormones and Behavior. 75, 100-110.

Kalinowski, S.T., Taper, M.L., Marshall, T.C., 2007. Revising how the computer program CERVUS accommodates genotyping error increases success in paternity assignment. Molecular Ecology. 16, 1099-1106.

Kappeler, P.M., 1999. Primate Socioecology: New Insights from Males. Naturwissenschaften. 85, 18 29.

Kappeler, P.M., 2012. Mate choice. In: Mitani, J.C., Call, J., Kappeler, P.M., Palombit, R., Silk, J. (Eds.), The Evolution of Primate Societies. University of Chicago Press, Chicago, pp. 367-386.

Kappeler, P.M., Cremer, S., Nunn, C.L., 2015. Sociality and health: impacts of sociality on disease susceptibility and transmission in animal and human societies. Philosophical Transactions of the Royal Society B: Biological Sciences. 370, 20140116.

Kappeler, P.M., Port, M., 2008. Mutual tolerance or reproductive competition? Patterns of reproductive skew among male redfronted lemurs (Eulemur fulvus rufus). Behavioral Ecology and Sociobiology. 62, 1477-1488.

Kappeler, P.M., van Schaik, C.P., 2002. Evolution of primate social systems. International journal of primatology, 23, 707-740. 
Keane, B., Waser, P.M., Creel, S.R., Creel, N.M., Elliott, L.F., Minchella, D.J., 1994. Subordinate reproduction in dwarf mongooses. Animal Behaviour. 47, 65-75.

Keller, L., Reeve, H.K., 1994. Partitioning of reproduction in animal societies. Trends in Ecology \& Evolution. 9, 98-102.

Kern, J.M., Radford, A.N., 2016. Social-bond strength influences vocally mediated recruitment to mobbing. Biology letters. 12, 20160648.

Killick, R., Haynes, K., Eckley, I., Fearnhead, P., Lee, J., 2016. Package 'changepoint' Version 2.2.2. GitHub repository, https://github.com/gobbios/EloRating.

Kitchen, D.M., 2004. Alpha male black howler monkey responses to loud calls: Effect of numeric odds, male companion behaviour and reproductive investment. Animal Behaviour. 67, 125-139.

Kitchen, D.M., Cheney, D.L., Seyfarth, R.M., 2004. Factors mediating inter-group encounters in savannah baboons (Papio cynocephalus ursinus). Behaviour. 141, 197-218.

Kitchen, D.M., Seyfarth, R.M., Fischer, J., Cheney, D.L., 2003. Loud calls as indicators of dominance in male baboons (Papio cynocephalus ursinus). Behavioral Ecology and Sociobiology. 53, 374 384.

Koenig, A., 2002. Competition for ressources and its behavioral consequences among female primates. International Journal of Primatology. 23, 759-784.

Koenig, B., 1994. Fitness effects of communal rearing in house mice: the role of relatedness versus familiarity. Animal Behavior. 48, 1449-1457.

Kopp, G.H., 2015. Gene Flow Dynamics in Baboons - The Influence of Social Systems. Georg-August-Universität Göttingen, Germany.

Kopp, G.H., Fischer, J., Patzelt, A., Roos, C., Zinner, D., 2015. Population genetic insights into the social organization of Guinea baboons (Papio papio): Evidence for female-biased dispersal. American Journal of Primatology. 77, 878-889.

Korstjens, A.H., Lehmann, J., Dunbar, R.I.M., 2010. Resting time as an ecological constraint on primate biogeography. Animal Behaviour. 79, 361-374.

Krause, J., Ruxton, G.D., 2002. Living in groups. Oxford University Press.

Krebs, J.R., Davies, N.B., 1993. An introduction to behavioural ecology. Blackwell Scientific Publications, Cambridge, MA, USA.

Krutzen, M., Sherwin, W.B., Connor, R.C., Barre, L.M., Van de Casteele, T., Mann, J., Brooks, R., 2003. Contrasting relatedness patterns in bottlenose dolphins (Tursiops sp.) with different alliance strategies. Proceedings of the Royal Society B: Biological Sciences. 270, 497-502.

Kubenova, B., Konecna, M., Majolo, B., Smilauer, P., Ostner, J., Schülke, O., 2017. Triadic awareness predicts partner choice in male-infant-male interactions in Barbary macaques. Animal Cognition. 20, 221-232.

Kuester, J., Paul, A., Arnemann, J., 1995. Age-related and individual differences of reproductive success in male and female Barbary macaques (Macaca sylvanus). Primates. 36, 461-476.

Kulahci, I.G., Rubenstein, D.I., Ghazanfar, A.A., 2015. Lemurs groom-at-a-distance through vocal networks. Animal Behaviour. 110, 179-186.

Kulik, L., Muniz, L., Mundry, R., Widdig, A., 2012. Patterns of interventions and the effect of coalitions and sociality on male fitness. Molecular Ecology. 21, 699-714.

Kummer, H., 1968. Social organization of hamadryas baboons: A field study. Bibliotheca Primatologica, S. Karger, Basel, No. 6.

Kummer, H., 1997. In quest of the sacred baboon: a scientist's journey. Princeton University Press, Princeton, NJ, USA. 
Kummer, H., Götz, W., Angst, W., 1974. Triadic differentiation: An inhibitory process protecting pair bonds in baboons. Behaviour. 49, 62-87.

Kutsukake, N., Nunn, C.L., 2006. Comparative tests of reproductive skew in male primates: The roles of demographic factors and incomplete control. Behavioral Ecology and Sociobiology. 60, 695706.

Kutsukake, N., Suetsugu, N., Hasegawa, T., 2006. Pattern, distribution, and function of greeting behavior among black-and-white colobus. International Journal of Primatology. 27, 1271-1291.

Langergraber, K., Mitani, J., Vigilant, L., 2009. Kinship and social bonds in female chimpanzees (Pan troglodytes). American Journal of Primatology. 71, 840-851.

Langergraber, K.E., Mitani, J.C., Vigilant, L., 2007. The limited impact of kinship on cooperation in wild chimpanzees. Proceedings of the National Academy of Sciences. 104, 7786-7790.

Langergraber, K.E., Mitani, J.C., Watts, D.P., Vigilant, L., 2013. Male-female socio-spatial relationships and reproduction in wild chimpanzees. Behavioral Ecology and Sociobiology. 67, 861-873.

Langergraber, K.E., Watts, D.P., Vigilant, L., Mitani, J.C., 2017. Group augmentation, collective action, and territorial boundary patrols by male chimpanzees. Proceedings of the National Academy of Sciences. 114, 7337-7342.

Launhardt, K., Borries, C., Hardt, C., Epplen, J.T., Winkler, P., 2001. Paternity analysis of alternative male reproductive routes among the langurs (Semnopithecus entellus) of Ramnagar. Animal Behaviour. 61, 53-64.

le Roux, A., Beehner, J.C., Bergman, T.J., 2011. Female philopatry and dominance patterns in wild geladas. American Journal of Primatology. 73, 422-430.

Leadbeater, E., Chittka, L., 2009. Bumble-bees learn the value of social cues through experience. Biology Letters. 5, 310-312.

Lehmann, J., Korstjens, A.H., Dunbar, R.I.M., 2007. Group size, grooming and social cohesion in primates. Animal Behaviour. 74, 1617-1629.

Lehmann, J., Majolo, B., McFarland, R., 2016. The effects of social network position on the survival of wild Barbary macaques, Macaca sylvanus. Behavioral Ecology. 27, 20-28.

Leigh, S.R., Setchell, J.M., Charpentier, M., Knapp, L.A., Wickings, E.J., 2008. Canine tooth size and fitness in male mandrills (Mandrillus sphinx). Journal of Human Evolution. 55, 75-85.

Li, C.C., Weeks, D.E., Chakravarti, A., 1993. Similarity of DNA fingerprints due to chance and relatedness. Human Heredity. 43, 45-52.

Li, X.Y., Kokko, H., 2019. Sex-biased dispersal: a review of the theory. Biological Reviews. 94, 721736.

Lukas, D., Clutton-brock, T.H., 2014. Costs of mating competition limit male lifetime breeding success in polygynous mammals. Proceedings of the Royal Society B: Biological Sciences. 281, 20140418.

Lukas, D., Clutton-Brock, T.H., 2013. The Evolution of Social Monogamy in Mammals. Science. 341, $526-530$.

Lukas, D., Reynolds, V., Boesch, C., Vigilant, L., 2005. To what extent does living in a group mean living with kin? Molecular Ecology. 14, 2181-2196.

Lynch, M., Ritland, K., 1999. Estimation of pairwise relatedness with molecular markers. Genetics. 152, 1753-1766.

Lynch Alfaro, J., 2008. Scream-embrace displays in wild black-horned capuchin monkeys. American Journal of Primatology. 70, 551-559.

Maciej, P., Patzelt, A., Ndao, I., Hammerschmidt, K., Fischer, J., 2013. Social monitoring in a multilevel society: A playback study with male Guinea baboons. Behavioral Ecology and Sociobiology. 67, 61-68. 
Majolo, B., Lehmann, J., De Bortoli Vizioli, A., Schino, G., 2012. Fitness-related benefits of dominance in primates. American Journal of Physical Anthropology. 147, 652-660.

Markham, A.C., Alberts, S.C., Altmann, J., 2012. Intergroup conflict: Ecological predictors of winning and consequences of defeat in a wild primate population. Animal Behaviour. 84, 399-403.

Markham, A.C., Lonsdorf, E. V., Pusey, A.E., Murray, C.M., 2015. Maternal rank influences the outcome of aggressive interactions between immature chimpanzees. Animal Behaviour. 100, 192198.

Massen, J.J.M., Sterck, E.H.M., Vos, H. De, 2010. Close social associations in animals and humans: functions and mechanisms of friendship. Behaviour. 147, 1379-1412.

Massen, J.J.M., Vries, A.M.O., 2012. Male mating tactics in captive rhesus macaques (Macaca mulatta): The Influence of dominance, markets, and relationship quality. International Journal of Primatology. 33, 73-92.

Matoba, T., Kutsukake, N., Hasegawa, T., 2013. Head rubbing and licking reinforce social bonds in a group of captive African lions, Panthera leo. PLoS ONE. 8, 1-11.

McCullagh, P., Nelder, J.A., 1989. Generalized Linear Models, 2nd ed, The American Statistician. Chapman and Hall, London.

McFarland, R., Fuller, A., Hetem, R.S., Mitchell, D., Maloney, S.K., Henzi, S.P., Barrett, L., 2015. Social integration confers thermal benefits in a gregarious primate. Journal of Animal Ecology. 84, 871-878.

McFarland, R., Henzi, S.P., Barrett, L., Wanigaratne, A., Coetzee, E., Fuller, A., Hetem, R.S., Mitchell, D., Maloney, S.K., 2016. Thermal consequences of increased pelt loft infer an additional utilitarian function for grooming. American Journal of Primatology. 78, 456-461.

McFarland, R., Majolo, B., 2013. Coping with the cold: predictors of survival in wild Barbary macaques, Macaca sylvanus. Biology Letters. 9, 20130428.

McFarland, R., Murphy, D., Lusseau, D., Henzi, S.P., Parker, J.L., Pollet, T. V, Barrett, L., 2017. The 'strength of weak ties' among female baboons: fitness-related benefits of social bonds. Animal Behaviour. 126, 101-106.

Melis, A.P., Hare, B., Tomasello, M., 2006. Engineering cooperation in chimpanzees: tolerance constraints on cooperation. Animal Behaviour. 72, 275-286.

Micheletta, J., Waller, B.M., Panggur, M.R., Neumann, C., Duboscq, J., Agil, M., Engelhardt, A., 2012. Social bonds affect anti-predator behaviour in a tolerant species of macaque, Macaca nigra. Proceedings of the Royal Society B: Biological Sciences. 279, 4042-4050.

Milligan, B.G., 2003. Maximum-Likelihood Estimation of Relatedness. Genetics. 163, 1153-1167.

Minge, C., Berghänel, A., Schülke, O., Ostner, J., 2016. Patterns and consequences of male-infant relationships in wild Assamese macaques (Macaca assamensis). International Journal of Primatology. 37, 350-370.

Mitani, J.C., 2009. Male chimpanzees form enduring and equitable social bonds. Animal Behaviour. 77, 633-640.

Mitani, J.C., Call, J., Kappeler, P.M., Palombit, R.A., Silk, J.B., 2012. The evolution of primate societies. University of Chicago Press, London Chicago.

Mitani, J.C., Gros-louis, J., Manson, J.H., 1996. Number of males in primate groups: Comparative tests of competing hypotheses. American Journal of Primatology. 38, 315-332.

Mitani, J.C., Watts, D.P., Amsler, S.J., 2010. Lethal intergroup aggression leads to territorial expansion in wild chimpanzees. Current Biology. 20, 507-508.

Modolo, L., Martin, R.D., 2008. Reproductive success in relation to dominance rank in the absence of prime-age males in Barbary macaques. American Journal of Primatology. 70, 26-34. 
Moscovice, L.R., Di Fiore, A., Crockford, C., Kitchen, D.M., Wittig, R.M., Seyfarth, R.M., Cheney, D.L., 2010. Hedging their bets? Male and female chacma baboons form friendships based on likelihood of paternity. Animal Behaviour. 79, 1007-1015.

Moscovice, L.R., Heesen, M., Di Fiore, A., Seyfarth, R.M., Cheney, D.L., 2009. Paternity alone does not predict long-term investment in juveniles by male baboons. Behavioral Ecology and Sociobiology. 63, 1471-1482.

Mosser, A., Packer, C., 2009. Group territoriality and the benefits of sociality in the African lion, Panthera leo. Animal Behaviour. 78, 359-370.

Newton-Fisher, N.E., Thompson, M.E., Reynolds, V., Boesch, C., Vigilant, L., 2010. Paternity and social rank in wild chimpanzees (Pan troglodytes) from the Budongo forest, Uganda. American Journal of Physical Anthropology. 142, 417-428.

Nguyen, N., Van Horn, R.C., Alberts, S.C., Altmann, J., 2009. "Friendships" between new mothers and adult males: Adaptive benefits and determinants in wild baboons (Papio cynocephalus). Behavioral Ecology and Sociobiology. 63, 1331-1344.

Noë, R., 1986. Lasting alliances among adult male savannah baboons. In: Else, J.G., Lee, P.C. (Eds.), Primate Ontogeny, Cognition and Social Behaviour. Cambridge University Press, Cambridge, pp. 381-392.

Noë, R., 1992. Alliance formation among male baboons: shopping for profitable partners. In: Harcourt, A., de Waal, F.B.M. (Eds.), Coatitions and Alliances in Humans and Other Animals. Oxford University Press, Oxford, UK, pp. 285-322.

Noë, R., Sluijter, A.A., 1990. Reproductive tactics of male savanna baboons. Behaviour. 113, 117-69.

Nonacs, P., 2000. Measuring and Using Skew in the Study of Social Behavior and Evolution. The American Naturalist. 156, 577-589.

Nonacs, P., 2003. Measuring the reliability of skew indices: is there one best index? Animal Behaviour. 65, 615-627.

Nunn, C.L., 1999a. The number of males in primate social groups: a comparative test of the sociecological model. Behavioral Ecology and Sociobiology. 46, 1-13.

Nunn, C.L., 1999b. The evolution of exaggerated sexual swellings in primates and the graded-signal hypothesis. Animal Behaviour. 58, 229-246.

Ostner, J., Kappeler, P., Heistermann, M., 2008a. Androgen and glucocorticoid levels reflect seasonally occurring social challenges in male redfronted lemurs (Eulemur fulvus rufus). Behavioral Ecology and Sociobiology. 62, 627-638.

Ostner, J., Kappeler, P.M., 2004. Male life history and the unusual adult sex ratios of redfronted lemur, Eulemur fulvus rufus, groups. Animal Behaviour. 67, 249-259.

Ostner, J., Nunn, C.L., Schülke, O., 2008b. Female reproductive synchrony predicts skewed paternity across primates. Behavioral Ecology. 19, 1150-1158.

Ostner, J., Schülke, O., 2014. The evolution of social bonds in primate males. Behaviour. 151, 1-36.

Ostner, J., Schülke, O., 2018. Linking sociality to fitness in primates: A call for mechanisms. Advances in the Study of Behavior. 50, 127-175.

Ostner, J., Vigilant, L., Bhagavatula, J., Franz, M., Schülke, O., 2013. Stable heterosexual associations in a promiscuous primate. Animal Behaviour. 86, 623-631.

Packer, C., Gilbert, D.A., Pusey, A.E., O’Brien, S.J., 1991. A molecular genetic analysis of kinship and cooperation in African lions. Nature. 351, 562-565.

Packer, C., Ruttan, L., 1998. The evolution of cooperative hunting. The American Journal of Sociology. 132, 159-198.

Packer, C., Scheel, D., Pusey, A.E., 1990. Why lions form groups: food is not enough. The American Naturalist. 136, 1-19. 
Palagi, E., 2006. Social play in bonobos (Pan paniscus) and chimpanzees (Pan troglodytes): Implications for natural social systems and interindividual relationships. American Journal of Physical Anthropology. 129, 418-426.

Palombit, R.A., 1999. Infanticide and the evolution of pair bonds in nonhuman primates. Evolutionary Anthropology. 7, 117-129.

Palombit, R.A., Seyfarth, R.M., Cheney, D.L., 1997. The adaptive value of "friendships" to female baboons: experimental and observational evidence. Animal Behaviour. 54, 599-614.

Pappano, D.J., 2013. The reproductive trajectories of bachelor geladas. Doctoral dissertation, The University of Michigan, USA.

Pappano, D.J., Snyder-Mackler, N., Bergman, T.J., Beehner, J.C., 2012. Social "predators" within a multilevel primate society. Animal Behaviour. 84, 653-658.

Patzelt, A., 2013. The Social System of Guinea Baboons ( Papio papio ) With a Focus on Male-Male Relationships. Doctoral dissertation, Georg-August-Universität Göttingen, Germany.

Patzelt, A., Kopp, G.H., Ndao, I., Kalbitzer, U., Zinner, D., Fischer, J., 2014. Male tolerance and malemale bonds in a multilevel primate society. Proceedings of the National Academy of Sciences. $111,14740-14745$.

Paul, A., Kuester, J., Arnemann, J., 1996. The sociobiology of male-infant interactions in Barbary macaques, Macaca sylvanus. Animal Behaviour. 51, 155-170.

Peláez, F., 1982. Greeting movements among adult males in a colony of baboons: Papio hamadryas, P. cynocephalus and their hybrids. Primates. 23, 233-244.

Perry, S., 1996. Intergroup encounters in wild white-faced capuchins (Cebus capucinus). International Journal of Primatology. 17, 309-330.

Perry, S., 1998. Male-male social relationships in wild white-faced capuchins, Cebus capucinus. Behaviour. 135, 139-172.

Perry, S., Barrett, C.H., Manson, J.H., 2004. White-faced capuchin monkeys show triadic awareness in their choice of allies. Animal Behaviour. 67, 165-170.

Pew, J., Muir, P.H., Wang, J., Frasier, T.R., 2015. related: An R package for analysing pairwise relatedness from codominant molecular markers. Molecular Ecology Resources. 15, 557-561.

Pines, M., Chowdhury, S., Saunders, J., Swedell, L., 2015. The rise and fall of leader males in a multilevel society: Takeovers and tenures of male hamadryas baboons. American Journal of Primatology. 77, 44-55.

Pitnick, S., Pfennig, D.W., 2014. Brotherly love benefits females. Nature. 505, 626-7.

Pizzari, T., Gardner, A., 2012. The sociobiology of sex: Inclusive fitness consequences of inter-sexual interactions. Philosophical Transactions of the Royal Society B: Biological Sciences. 367, 2314-2323.

Plavcan, J.M., 1993. Canine size and shape in male anthropoid primates. American Journal of Physical Anthropology. 92, 201-216.

Plavcan, J.M., van Schaik, C.P., Kappeler, P.M., 1995. Competition, coalitions and canine size in primates. Journal of Human Evolution. 28, 245-276.

Pope, T.R., 1990. The reproductive consequences of male cooperation in the red howler monkey: paternity exclusion in multi-male and single-male troops using genetic markers. Behavioral Ecology and Sociobiology. 27, 439-446.

Port, M., Johnstone, R.A., 2013. Facing the crowd: intruder pressure, within-group competition, and the resolution of conflicts over group-membership. Ecology and evolution. 3, 1209-1218.

Port, M., Johnstone, R.A., Kappeler, P.M., 2010. Costs and benefits of multi-male associations in redfronted lemurs (Eulemur fulvus rufus). Biology Letters. 6, 620-622. 
Port, M., Kappeler, P.M., 2010. The utility of reproductive skew models in the study of male primates, a critical evaluation. Evolutionary Anthropology. 19, 46-56.

Port, M., Schülke, O., Ostner, J., 2018. Reproductive tolerance in male primates: Old paradigms and new evidence. Evolutionary Anthropology. 27, 107-120.

Qi, X., Huang, K., Fang, G., Grueter, C.C., Dunn, D.W., Li, Y., Ji, W., Wang, X., Wang, R., Garber, P.A., Li, B., 2017. Male cooperation for breeding opportunities contributes to the evolution of multilevel societies. Proceedings of the Royal Society B: Biological Sciences. 284, 20171480.

Queller, D.C., Goodnight, K.F., 1989. Estimating Relatedness Using Genetic Markers. Evolution. 43, 258-275.

Queller, D.C., Zacchi, F., Cervo, R., Turillazzi, S., Henshaw, M.T., Santorelli, L.A., Strassmann, J.E., 2000. Unrelated helpers in a social insect. Nature. 405, 784-787.

Quinn, G.P., Keough, M.J., 2002. Experimental design and data analysis for biologists. Cambridge University Press, Cambridge.

R Development Core Team, 2018. R: a language and environment for statistical computing. R Foundation for Statistical Computing. Vienna, Austria.

Radford, A.N., 2008. Type of threat influences postconflict allopreening in a social bird. Current Biology. 18, 114-115.

Rankin, D.J., 2011. Kin selection and the evolution of sexual conflict. Journal of Evolutionary Biology. $24,71-81$.

Rappaport, R.A., 1979. The obvious aspects of ritual. In: Ecology, Meaning, and Religion. North Atlantic Books, Berkeley, pp. 173-219.

Rathke, E.M., Berghänel, A., Bissonnette, A., Ostner, J., Schülke, O., 2017. Age-dependent change of coalitionary strategy in male Barbary macaques. Primate Biology. 4, 1-7.

Reed, C., O’Brien, T.G., Kinnaird, M.F., 1997. Male social behavior and dominance hierarchy in the Sulawesi Crested Black Macaque (Macaca nigra). International Journal of Primatology. 18, 247260.

Reeve, H.K., Emlen, S.T., Keller, L., 1998. Reproductive sharing in animal societies: reproductive incentives or incomplete control by dominant breeders? Behavioral Ecology. 9, 267-278.

Reeve, H.K., Keller, L., 1998. Reproductive skew: disentangling concessions from control. Trends in Ecology \& Evolution. 13, 458-459.

Reeve, H.K., Ratnieks, F.L.W., 1993. Queen-queen conflict in polygynous societies: mutual tolerance and reproductive skew. In: Keller, L. (Ed.), Queen Number and Sociality in Insects. Oxford University Press, Oxford, pp. 45-85.

Richter, C., Heesen, M., Nenadic, O., Ostner, J., Schülke, O., 2016. Males matter: Increased home range size is associated with the number of resident males after controlling for ecological factors in wild Assamese macaques. American Journal of Physical Anthropology. 159, 52-62.

Riehl, C., Strong, M.J., 2018. Stable social relationships between unrelated females increase individual fitness in a cooperative bird. Proceedings of the Royal Society B: Biological Sciences. 285, 20180130.

Riley, E.P., Sagnotti, C., Carosi, M., Oka, N.P., 2014. Socially tolerant relationships among wild male moor macaques (Macaca maura). Behaviour. 151, 1021-1044.

Ritland, K., 1996. Estimators for pairwise relatedness and individual inbreeding coefficients. Genetical Research. 67, 175-185.

Robbins, M.M., 1995. A demographic analysis of male life history and social structure of mountain gorillas. Behaviour. 132, 21-47.

Roeder, A.D., Archer, F.I., Poinar, H.N., Morin, P.A., 2004. A novel method for collection and preservation of faeces for genetic studies. Molecular Ecology Notes. 4, 761-764. 
Rose, L.M., 1994. Benefits and costs of resident males to females in white-faced capuchins, Cebus capucinus. American Journal of Primatology. 32, 235-248.

Rosenbaum, S., Vigilant, L., Kuzawa, C.W., Stoinski, T.S., 2018. Caring for infants is associated with increased reproductive success for male mountain gorillas. Scientific Reports. 8, 15223.

Rossano, M.J., 2012. The essential role of ritual in the transmission and reinforcement of social norms. Psychological Bulletin. 138, 529-549.

Rossano, M.J., 2015. The evolutionary emergence of costly rituals. PaleoAnthropology. 78-100.

Rowell, T.E., 1988. Beyond the one-male group. Behaviour. 104, 189-201.

RStudio Team, 2018. RStudio: Integrated Development for R. RStudio, Inc. Boston, Massachusetts.

Rubenstein, D.I., 1978. On predation, competition, and the advantages of group living. In: Bateson, P. (Ed.), Social behavior. Springer US, Boston, MA, pp. 205-231.

Rubenstein, D.I., 1994. The ecology of female social behavior in horses, zebras, and asses. In: Jarman, P., Rossiter, A. (Eds.), Animal societies: Individuals, interactions, and organization. Physiology and Ecology Japan: Kyoto University Press, Kyoto, pp. 13-28.

Rubenstein, D.I., Hack, M., 2004. Natural and sexual selection and the evolution of multi-level societies: insights from zebras with comparisons to primates. In: Kappeler, P.M., van Schaik, C.P. (Eds.), Sexual selection in primates: New and comparative perspectives, Cambridge University Press, pp. 266-279.

Saayman, G.S., 1971. Behaviour of the adult males in a troop of free-ranging chacma baboons (papio ursinus). Folia Primatologica. 15, 36-57.

Sabbatini, G., De Bortoli Vizioli, A., Visalberghi, E., Schino, G., 2012. Food transfers in capuchin monkeys: an experiment on partner choice. Biology Letters. 8, 757-759.

Samuni, L., Preis, A., Deschner, T., Crockford, C., Wittig, R.M., 2018a. Reward of labor coordination and hunting success in wild chimpanzees. Communications Biology. 1, 1-9.

Samuni, L., Preis, A., Mielke, A., Deschner, T., Wittig, R.M., Crockford, C., 2018b. Social bonds facilitate cooperative resource sharing in wild chimpanzees. Proceedings of the Royal Society B. 285, 20181643.

Sánchez-Tójar, A., Schroeder, J., Farine, D.R., 2018. A practical guide for inferring reliable dominance hierarchies and estimating their uncertainty. Journal of Animal Ecology. 87, 594-608.

Satinoff, E., 2011. Behavioral thermoregulation in the cold. Comparative Physiology. 14, 481-505.

Scarry, C.J., 2013. Between-group contest competition among tufted capuchin monkeys, Sapajus nigritus, and the role of male resource defence. Animal Behaviour. 85, 931-939.

Scarry, C.J., 2017. Male resource defence during intergroup aggression among tufted capuchin monkeys. Animal Behaviour. 123, 169-178.

Schielzeth, H., 2010. Simple means to improve the interpretability of regression coefficients. Methods in Ecology and Evolution. 1, 103-113.

Schoof, V.A.M., Jack, K.M., 2014. Male social bonds: strength and quality among co-resident whitefaced capuchin monkeys (Cebus capucinus). Behaviour. 151, 963-992.

Schreier, A.L., Swedell, L., 2009. The fourth level of social structure in a multi-level society: Ecological and social functions of clans in Hamadryas Baboons. American Journal of Primatology. 71, 948-955.

Schülke, O., Bhagavatula, J., Vigilant, L., Ostner, J., 2010. Social bonds enhance reproductive success in male macaques. Current Biology. 20, 2207-2210.

Schülke, O., Heistermann, M., Ostner, J., 2014. Lack of evidence for energetic costs of mate-guarding in wild male Assamese macaques (Macaca assamensis). International Journal of Primatology. 35, 677-700. 
Schülke, O., Ostner, J., 2017. Male bonding. In: The International Encyclopedia of Primatology. John Wiley \& Sons, Inc., Hoboken, NJ, USA.

Setchell, J.M., Charpentier, M., Wickings, E.J., 2005. Sexual selection and reproductive careers in mandrills (Mandrillus sphinx). Behavioral Ecology and Sociobiology. 58, 474-485.

Shizuka, D., McDonald, D.B., 2012. A social network perspective on measurements of dominance hierarchies. Animal Behaviour. 83, 925-934.

Sicotte, P., 1993. Inter-group encounters and female transfer in mountain gorillas: Influence of group composition on male behavior. American Journal of Primatology. 30, 21-36.

Silk, J.B., 1994. Social relationships of male bonnet macaques: male bonding in a matrilineal society. Behaviour. 130, 271-291.

Silk, J.B., 2002. Using the 'F'-word in primatology. Behaviour. 139, 421-446.

Silk, J.B., 2007a. Social components of fitness in primate groups. Science. 317, 1347-1351.

Silk, J.B., 2007b. The adaptive value of sociality in mammalian groups. Philosophical Transactions of the Royal Society B: Biological Sciences. 362, 539-559.

Silk, J.B., Alberts, S.C., Altmann, J., 2003. Social bonds of female baboons enhance infant survival. Science. 302, 1231-1234.

Silk, J.B., Alberts, S.C., Altmann, J., 2006a. Social relationships among adult female baboons (Papio cynocephalus) II. Variation in the quality and stability of social bonds. Behavioral Ecology and Sociobiology. 61, 197-204.

Silk, J.B., Alberts, S.C., Altmann, J., Cheney, D.L., Seyfarth, R.M., 2012. Stability of partner choice among female baboons. Animal Behaviour. 83, 1511-1518.

Silk, J.B., Altmann, J., Alberts, S.C., 2006b. Social relationships among adult female baboons (papio cynocephalus) I. Variation in the strength of social bonds. Behavioral Ecology and Sociobiology. 61, 183-195.

Silk, J.B., Beehner, J.C., Bergman, T.J., Crockford, C., Engh, A.L., Moscovice, L.R., Wittig, R.M., Seyfarth, R.M., Cheney, D.L., 2009. The benefits of social capital: close social bonds among female baboons enhance offspring survival. Proceedings of the Royal Society B: Biological Sciences. 276, 3099-3104.

Silk, J.B., Beehner, J.C., Bergman, T.J., Crockford, C., Engh, A.L., Moscovice, L.R., Wittig, R.M., Seyfarth, R.M., Cheney, D.L., 2010a. Female chacma baboons form strong, equitable, and enduring social bonds. Behavioral Ecology and Sociobiology. 64, 1733-1747.

Silk, J.B., Beehner, J.C., Bergman, T.J., Crockford, C., Engh, A.L., Moscovice, L.R., Wittig, R.M., Seyfarth, R.M., Cheney, D.L., 2010b. Strong and consistent social bonds enhance the longevity of female baboons. Current Biology. 20, 1359-1361.

Silk, J.B., Cheney, D., Seyfarth, R., 2013. A practical guide to the study of social relationships. Evolutionary Anthropology. 22, 213-225.

Silk, J.B., Roberts, E.R., Barrett, B.J., Patterson, S.K., Strum, S.C., 2017. Female-male relationships influence the form of female-female relationships in olive baboons, Papio anubis. Animal Behaviour. 131, 89-98.

Silk, J.B., Seyfarth, R.M., Cheney, D.L., 2018. Quality versus quantity: do weak bonds enhance the fitness of female baboons? Animal Behaviour. 140, 207-211.

Smith, J.E., 2014. Hamilton's legacy: kinship, cooperation and social tolerance in mammalian groups. Animal Behaviour. 92, 291-304.

Smith, J.E., Powning, K.S., Dawes, S.E., Estrada, J.R., Hopper, A.L., Piotrowski, S.L., Holekamp, K.E., 2011. Greetings promote cooperation and reinforce social bonds among spotted hyaenas. Animal Behaviour. 81, 401-415. 
Smuts, B., Watanabe, J.M., 1990. Social relationships and ritualized greetings in adult male baboons (Papio cynocephalus anubis). International Journal of Primatology. 11, 147-172.

Smuts, B.B., Smuts, R.W., 1993. Male-aggression and sexual coercion of females in nonhuman-primates and other mammals - Evidence and theoretical implications. Advances in the Study of Behavior. 22, 1-63.

Snyder-Mackler, N., Alberts, S.C., Bergman, T.J., 2012. Concessions of an alpha male? Cooperative defence and shared reproduction in multi-male primate groups. Proceedings of the Royal Society B: Biological Sciences. 279, 3788-3795.

Soltis, J., Thomsen, R., Takenaka, O., 2001. The interaction of male and female reproductive strategies and paternity in wild Japanese macaques, Macaca fuscata. Animal Behavior. 62, 485-494.

Sosis, R., Bressler, E.R., 2003. Signaling theory of religion. Cross-cultural research. 37, 211-239.

Städele, V., Pines, M., Swedell, L., Vigilant, L., 2016. The ties that bind: Maternal kin bias in a multilevel primate society despite natal dispersal by both sexes. American Journal of Primatology. 78, 731-744.

Städele, V., Roberts, E.R., Barrett, B.J., Strum, S.C., Vigilant, L., Silk, J.B., 2019. Male-female relationships in olive baboons (Papio anubis): Parenting or mating effort? Journal of Human Evolution journal. 127, 81-92.

Städele, V., Van Doren, V., Pines, M., Swedell, L., Vigilant, L., 2015. Fine-scale genetic assessment of sex-specific dispersal patterns in a multilevel primate society. Journal of Human Evolution. 78, $103-113$.

Stanford, C.B., 1998. Predation and male bonds in primate societies. Behaviour. 135, 513-533.

Stanton, M.A., Mann, J., 2012. Early social networks predict survival in wild bottlenose dolphins. PloS one. 7, e 47508 .

Steenbeek, R., Piek, R.C., Van Buul, M., van Hooff, J.A.R.A.M., 1999. Vigilance in wild Thomas's langurs (Presbytis thomasi): The importance of infanticide risk. Behavioral Ecology and Sociobiology. 45, 137-150.

Sterck, E.H.M., Watts, D.P., van Schaik, C.P., 1997. The evolution of female social relationships in nonhuman primates. Behavioral Ecology and Sociobiology. 41, 291-309.

Stoinski, T.S., Rosenbaum, S., Ngaboyamahina, T., Vecellio, V., Ndagijimana, F., Fawcett, K., 2009. Patterns of male reproductive behaviour in multi-male groups of mountain gorillas: examining theories of reproductive skew. Behaviour. 146, 1193-1215.

Street, S.E., Cross, C.P., Brown, G.R., 2016. Exaggerated sexual swellings in female nonhuman primates are reliable signals of female fertility and body condition. Animal Behaviour. 112, 203-212.

Struhsaker, T.T., 1969. Correlates of ecology and social organization among African cercopithecines. Folia primatologica. 11, 80-118.

Sundaresan, S.R., Fischhoff, I.R., Rubenstein, D.I., 2007. Male harassment influences female movements and associations in Grevy's zebra (Equus grevyi). Behavioral Ecology. 18, 860-865.

Swedell, L., 2000. Two takeovers in wild hamadryas baboons. Folia Primatologica. 71, 169-172.

Swedell, L., 2002. Affiliation among females in wild hamadryas baboons (Papio hamadryas hamadryas). International Journal of Primatology. 23, 1205-1226.

Swedell, L., 2006. Strategies of sex and survival in hamadryas baboons: Through a female lens, primate field studies. Pearson Prentice Hall, Upper Saddle River, NJ.

Swedell, L., 2011. African Papionins: Diversity of social organisation and ecological flexibility. In: Primates in perspective. Oxford University Press, London, pp. 241-277.

Swedell, L., Leedom, L., Saunders, J., Pines, M., 2014. Sexual conflict in a polygynous primate: costs and benefits of a male-imposed mating system. Behavioral ecology and sociobiology. 68, 263-273. 
Swedell, L., Plummer, T., 2012. A papionin multilevel society as a model for hominin social evolution. International Journal of Primatology. 33, 1165-1193.

Swedell, L., Saunders, J., 2006. Infant Mortality, Paternity Certainty, and Female Reproductive Strategies in Hamadryas Baboons. In: Swedell L., Leigh S.R. (Eds.), Reproduction and fitness in baboons: Behavioral, ecological, and life history perspectives. Developments in Primatology: Progress and Prospects. Springer, Boston, MA, pp. 19-51.

Swedell, L., Schreier, A., 2009. Male aggression towards females in hamadryas baboons: Conditioning, coercion, and control. In: Martin, N.M., Wrangham, R.W. (Eds.), Sexual coercion in primates and humans: an evolutionary perspective on male aggression against females. Harvard UniversityPress, USA, pp. 244-268.

Swedell, L., Tesfaye, T., 2003. Infant mortality after takeovers in wild Ethiopian hamadryas baboons. American Journal of Primatology. 60, 113-118.

Talebi, M.G., Beltrão-Mendes, R., Lee, P.C., 2009. Intra-community coalitionary lethal attack of an adult male southern muriqui (Brachyteles arachnoides). American Journal of Primatology. 71, $860-867$.

Teichroeb, J.A., Ting, N., Wikberg, E.C., Sicotte, P., 2014. Factors influencing male affiliation and coalitions in a species with male dispersal and intense male-male competition, Colobus vellerosus. Behaviour. 151, 1045-1066.

Terrien, J., Perret, M., Aujard, F., 2011. Behavioral thermoregulation in mammals: a review. Frontiers in Bioscience. 16, 1428-1444.

Thompson, N.A., 2019. Understanding the links between social ties and fitness over the life cycle in primates. Behaviour. [https://doi.org/10.1163/1568539X-00003552].

Thompson, N.A., Cords, M., 2018. Stronger social bonds do not always predict greater longevity in a gregarious primate. Ecology and Evolution. 8, 1604-1614.

Tiddi, B., Aureli, F., Polizzi Di Sorrentino, E., Janson, C.H., Schino, G., 2011. Grooming for tolerance? Two mechanisms of exchange in wild tufted capuchin monkeys. Behavioral Ecology. 22, 663-669.

Trivers, L.R., 1972. Parental investiment and sexual seletion. In: Campbell, B. (Ed.), Sexual selection and the descent of man, Aldine DeGruyter, New York, USA, pp. 136-179.

van Hooff, J.A.R.A.M., van Schaik, C.P., 1994. Male bonds: Afilliative relationships among nonhuman primate males. Behaviour. 130, 309-337.

van Hooff, J. a R. a M., 2000. Relationships among nonhuman primate males: a deductive framework. In: Kappeler, P.M. (Ed.), Primate Males. Cambridge University Press, Cambridge.

Van Oosterhout, C., Hutchinson, W.F., Wills, D.P.M., Shipley, P., 2004. MICRO-CHECKER: Software for identifying and correcting genotyping errors in microsatellite data. Molecular Ecology Notes. 4, 535-538.

van Schaik, C.P., 1983. Why are diurnal primates living in groups? Behaviour. 87, 120-144.

van Schaik, C.P., 1989. The ecology of social relationships amongst female primates. In: Standen, V., Foley, R.A., (Eds.), Comparative socioecology: The behavioural ecology of humans and other mammals, Blackwell Scientific Publications, USA, pp. 195-218.

van Schaik, C.P., Hörstermann, M., 1994. Predation risk and the number of adult males in a primate group: a comparative test. Behavioral Ecology and Sociobiology. 35, 261-272.

van Schaik, C.P., Kappeler, P.M., 1997. Infanticide risk and the evolution of male-female association in primates. Proceedings of the Royal Society of London. Series B: Biological Sciences. 264, 16871694.

van Schaik, C.P., Pandit, S.A., Vogel, E.R., 2004. A model for within-group coalitionary aggression among males. Behavioral Ecology and Sociobiology. 57, 101-109. 
van Schaik, C.P., Pandit, S.A., Vogel, E.R., 2006. Toward a general model for male-male coalitions in primate groups. In: Kappeler, P.M., van Schaik, C.P. (Eds.), Cooperation in primates and humans: Mechanisms and evolution, Springer, Berlin, Heidelberg, pp. 151-171.

van Schaik, C.P., van Hooff, J.A.R.A.M., 1983. On the ultimate causes of primate social systems. Behaviour. $85,91-117$.

van Schaik, C.P., van Noordwijk, M.A., 1989. The special role of male Cebus monkeys in predation avoidance and its effect on group composition. Behavioral Ecology and Sociobiology. 24, 265-276.

Vasey, P.L., Sommer, V., 2006. Homosexual behaviour in animals, topics, hypothesis and research trajectories. In: Vasey, P.L., Sommer, V. (Eds.), Homosexual behaviour in animals, an evolutionary perspective. Cambridge University Press, Cambridge, pp. 3-42.

Wang, E., Milton, K., 2003. Intragroup social relationships of male Alouatta palliata on Barro Colorado Island, Republic of Panama. International Journal of Primatology. 24, 1227-1243.

Wang, J., 2002. An estimator for pairwise relatedness using molecular markers. Genetics. 160, 12031215.

Wang, J., 2007. Triadic IBD coefficients and applications to estimating pairwise relatedness. Genetical Research. 89, 135-153.

Wang, J., 2011. Coancestry: A program for simulating, estimating and analysing relatedness and inbreeding coefficients. Molecular Ecology Resources. 11, 141-145.

Wang, J., 2017. Estimating pairwise relatedness in a small sample of individuals. Heredity. 119, 302313.

Watson-Jones, R.E., Legare, C.H., 2016. The social functions of group rituals. Current Directions in Psychological Science. 25, 42-46.

Watts, D.P., 1998. Coalitionary mate guarding by male chimpanzees at Ngogo, Kibale National Park, Uganda. Behavioral Ecology and Sociobiology. 44, 43-55.

Watts, D.P., 2002. Reciprocity and Interchange in the Social Relationships of Wild Male Chimpanzees. Behaviour. 139, 343-370.

Weidt, A., Hofmann, S.E., König, B., 2008. Not only mate choice matters: fitness consequences of social partner choice in female house mice. Animal Behaviour. 75, 801-808.

Weingrill, T., Lycett, J.E., Barrett, L., Hill, R.A., Henzi, S.P., 2003. Male consortship behaviour in chacma baboons: the role of demographic factors and female conceptive probabilities. Behaviour. 140, 405-427.

Wey, T.W., Blumstein, D.T., 2012. Social attributes and associated performance measures in marmots: Bigger male bullies and weakly affiliating females have higher annual reproductive success. Behavioral Ecology and Sociobiology. 66, 1075-1085.

Whitham, J.C., Maestripieri, D., 2003. Primate rituals: The function of greetings between male Guinea Baboons. Ethology. 109, 847-859.

Wich, S.A., Assink, P.R., Sterck, E.H.M., 2004. Thomas langurs (Presbytis thomasi) discriminate between calls of young solitary versus older group-living males: A factor in avoiding infanticide? Behaviour. 141, 41-51.

Widdig, A., 2007. Paternal kin discrimination: the evidence and likely mechanisms. Biological Reviews. 82, 319-334.

Widdig, A., Bercovitch, F.B., Streich, W.J., Sauermann, U., Nürnberg, P., Krawczak, M., 2004. A longitudinal analysis of reproductive skew in male rhesus macaques. Proceedings of the Royal Society B: Biological Sciences. 271, 819-826.

Wild, G., Pizzari, T., West, S.A., 2011. Sexual conflict in viscous populations: The effect of the timing of dispersal. Theoretical Population Biology. 80, 298-316. 
Williams, G.C., 1966. Adaptation and natural selection: A critique of some current evolutionary thought (Vol. 61). Princeton University Press, Princeton, New Jersey.

Williams, J.M., Oehlert, G.W., Carlis, J. V., Pusey, A.E., 2004. Why do male chimpanzees defend a group range? Animal Behaviour. 68, 523-532.

Wilson, M.L., Kahlenberg, S.M., Wells, M., Wrangham, R.W., 2012. Ecological and social factors affect the occurrence and outcomes of intergroup encounters in chimpanzees. Animal Behaviour. 83, 263-268.

Wiszniewski, J., Corrigan, S., Beheregaray, L.B., Möller, L.M., 2012. Male reproductive success increases with alliance size in Indo-Pacific bottlenose dolphins (Tursiops aduncus). Journal of Animal Ecology. 81, 423-431.

Wittig, R.M., Crockford, C., Deschner, T., Langergraber, K.E., Ziegler, T.E., Zuberbu, K., Crockford, C., 2014. Food sharing is linked to urinary oxytocin levels and bonding in related and unrelated wild chimpanzees.

Wittig, R.M., Crockford, C., Lehmann, J., Whitten, P.L., Seyfarth, R.M., Cheney, D.L., 2008. Focused grooming networks and stress alleviation in wild female baboons. Hormones and Behavior. 54, $170-177$.

Wong, M.Y.L., Munday, P.L., Buston, P.M., Jones, G.P., 2004. Fasting or feasting in a fish social hierarchy. Current Biology. 18, 372-373.

Wooldridge, J.M., 2013. Introductory econometrics: a modern approach, 5th ed., Cengage Learning, Andover, UK.

Wrangham, R.W., 1980. An ecological model of female-bonded primate groups. Behaviour. 75, 262 300.

Wrangham, R.W., Rubenstein, D.I., 1986. Social evolution in birds and mammals. In: Rubenstein, D.I., Wrangham, R. (Eds.), Ecological aspects of social evolution: Birds and mammals. Princeton University Press, Princeton, NJ, pp. 452-470.

Wright, P.C., 1990. Patterns of paternal care in Primates. International Journal of Primatology. 11, 89-102.

Wright, S., 1921. Systems of mating. Genetics. 6, 111-178.

Wright, S., 1922. Coefficients of inbreeding and relationship. The American Naturalist. 56, 330-338.

Xia, D.P., Li, J.H., Garber, P.A., Matheson, M.D., Sun, B.H., Zhu, Y., 2013. Grooming reciprocity in male Tibetan macaques. American Journal of Primatology. 75, 1009-1020.

Xiang, Z.F., Yang, B.H., Yu, Y., Yao, H., Grueter, C.C., Garber, P.A., Li, M., 2014. Males collectively defend their one-male units against bachelor males in a multi-level primate society. American Journal of Primatology. 76, 609-617.

Xygalatas, D., Mitkidis, P., Fischer, R., Reddish, P., Skewes, J., Geertz, A.W., Roepstorff, A., Bulbulia, J., 2013. Extreme rituals promote prosociality. Psychological Science. 24, 1602-1605.

Yang, W.J., Maldonado-chaparro, A.A., Blumstein, D.T., 2016. A cost of being amicable in a hibernating mammal. Behavioral Ecology. 28, 11-19.

Young, C., Hähndel, S., Majolo, B., Schülke, O., Ostner, J., 2013. Male coalitions and female behaviour affect male mating success independent of dominance rank and female receptive synchrony in wild Barbary macaques. Behavioral Ecology and Sociobiology. 67, 1665-1677.

Young, C., Majolo, B., Heistermann, M., Schulke, O., Ostner, J., 2014a. Responses to social and environmental stress are attenuated by strong male bonds in wild macaques. Proceedings of the $\mathrm{Na}$ tional Academy of Sciences. 111, 18195-18200.

Young, C., Majolo, B., Schülke, O., Ostner, J., 2014b. Male social bonds and rank predict supporter selection in cooperative aggression in wild Barbary macaques. Animal Behaviour. 95, 23-32. 
Young, C., Ostner, J., Schülke, O., 2014c. How males form coalitions against group rivals and the Pandit/van Schaik coalition model. Behaviour. 151, 907-934.

Young, L.J., Campbell, N.L., Capuano, G.A., 1999. Analysis of overdispersed count data from singlefactor experiments: A comparative study. Journal of Agricultural, Biological, and Environmental Statistics. 4, 258-275.

Zhang, P., Watanabe, K., Li, B., Tan, C.L., 2006. Social organization of Sichuan snub-nosed monkeys (Rhinopithecus roxellana) in the Qinling Mountains, Central China. Primates. 47, 374-382.

Zipple, M.N., Grady, J.H., Gordon, J.B., Chow, L.D., Archie, E.A., Altmann, J., Alberts, S.C., 2017. Conditional fetal and infant killing by male baboons. Proceedings of the Royal Society B: Biological Sciences. 284, 20162561. 


\section{Table of Figures}

Figure 4.1 Distribution of the dyadic composite sociality index .38

Figure 4.2 Observed vs. expected male partner stability indices

Figure 4.3 Relatedness estimates by male relationship type 1 (friends vs. non-friends) . .40

Figure 4.4 Effect of bond strength on rate of coalitionary support...... .41

Figure 4.5 Effect of male bond strength on number of associated females. . .42

Figure 4.6 Effect of number of associated females on time spent socializing....... .42

Figure S4.1 Change point analysis performed on male-male approach rate .55

Figure S4.2 Male interactions with/proximity to primary male's females. . .56

Figure S4.3 Distribution of the dyadic composite sociality index for 2014 and 2015 .56

Figure S4.4 Randomized Elo-rating scores for party 6 and party 9 . .57

Figure S4.5 Hierarchy shape plots for party 6 and party 9. .58

Figure S4.6 Relatedness estimates by male relationship type 2 (primary/secondary vs. other dyads) ..59 


\section{Table of Tables}

Table S4.1a Average group composition 2014-2015

Table S4.1b Average group composition 2016-2017

Table S4.2a Sex/age category definitions.

Table S4.2b Tooth status category definitions

Table S4.3 Yearly data collection details from 2014 to 2017.

Table S4.4a Descriptive statistics for all behavioral components included in DSI .61

Table S4.4b Results from the Kendall's tau correlation test of DSI behavioral components.....

Table S4.5 List of 24 microsatellite loci...... .62

Table S4.6a Characteristics of microsatellite loci to estimate dyadic relatedness.

Table S4.6b Characteristics of microsatellite loci to estimate paternity. .64

Table S4.7a Descriptive statistics for DSI indices .66

Table S4.7b Descriptive statistics for bond strength and number of friends and secondary males......66

Table S4.8a Mean randomized Elo-rating scores (rElo) for party 6. . .66

Table S4.8b Mean randomized Elo-rating scores (rElo) for party 9. .66

Table S4.9 Results of reproductive skew analysis

Table S4.10 Results and details of paternity analysis

Table S4.11a Kinship and male relationship type 1 (friends vs. non-friends)......

Table S4.11b Kinship and male relationship type 2 (primary/secondary vs. other dyads)

Table S4.12 Coalitionary support and dyadic composite sociality index ......

Table S4.13a Weighted average of associated females and male sociality (4a)

Table S4.13b Weighted average of associated females and male sociality (4b)...... .70 
Table S4.14a Number of sired offspring and male sociality (5a) ............................................................

Table S4.14b Number of sired offspring and male sociality (5b) …………........................................

Table S4.15 Post-hoc test: time spent socializing with males and number of associated females ..........71

Table S4.16a Male ad libitum interactions with primary male's females (1a) ...........................................72

Table S4.16b Male focal interactions with primary male's females (1b) ...................................................72

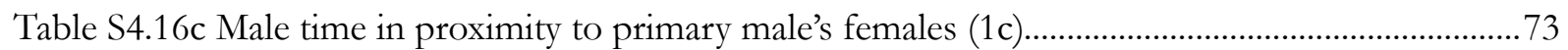




\section{Acknowledgments}

First and foremost I would like to thank my supervisor Julia Fischer for giving me the opportunity to embark on the long and challenging voyage of exploration and adventure that is the $\mathrm{PhD}$. Thank you for believing in me since I was a master student dreaming about observing wild primate behavior. My first field trip to Senegal was the realization of a long-time aspiration and the opportunity of continuing this experience with this $\mathrm{PhD}$ shaped my life and my knowledge in ways I could not have imagined. These years have brought countless challenges, many of which unexpected, and I want to thank you for supporting and trusting me with many responsibilities. The skills I have achieved under your supervision will be crucial in my future professional as well as personal life. I am also grateful to Oliver Schülke for having kindly accepted to review my dissertation and for his encouraging positivity and genuine interest throughout the project. I additionally want to thank Tanya Behne for having accepted to be part of my thesis committee and for her helpful contribution and feedback during our annual meetings. I further thank Julia Ostner, Christian Roos, and Annekathrin Schacht for agreeing to be members of my evaluation committee.

I want to thank my $\mathrm{PhD}$ graduate program "BeCog" as well as the "Research Training Group 2070" and the "Leibniz ScienceCampus Primate Cognition" to have provided funding and opportunities such as support for conference attendance, lab visits to other research groups, and many interesting workshops. A special thanks goes to Rebecca Jürgens and Christian Schloegl for their valuable help with the administration throughout my $\mathrm{PhD}$.

I am grateful to Holger Sennhenn-Reulen, Roger Mundry, and Christof Neumann for their valuable support with statistics, modeling, programming in $\mathrm{R}$ and always being available and helpful.

I want to thank Micheal and Andrea Heistermann for providing training and guidance with the hormone laboratory analysis. Even if this part of the project did not make into this thesis, the time working in your lab was incredibly valuable. Thank you for your precious help and competence and the nice chats about horses, life and handy-crafts.

Working in the field for the two years of my $\mathrm{PhD}$ data collection has been an immense source of challenges but also brought many thrilling and stimulating experiences. Most of all I want to thank all the field assistants (Mathieu, Franzi Wegdell, Lauriane, and Eréna) for patiently helping me to collect these valuable data and tenaciously following monkeys for fecal sample collection during so many long hours. Lauriane, a big and special thanks for all the time shared together in the field and back in Europe. It is rare to find an assistant so motivated and focused as you were and I am so glad this led to our friendship and, most of all, for all the fun in the office, the baboon conversations, and all the non-work chats in front of a chai latte and a latte macchiato. It was great to have someone with whom I could share the love and the interest for the baboons!! My Senegalese experience would not have been the same without the many local people that welcomed and supported me in the field, 
at camp, and in Tambacounda. A huge thank you goes to the Senegalese field assistants who accompanied and assisted me every day in the field. Faye, Nyaf, Sane, Dans, Diedhiou, M. Dieng and Sonko, thank you for the many kilometers walked and ran together following the baboons, for the many talks and laughs walking back to camp and for sharing funny cultural anecdotes and stories about traditions, family life, and the wilderness. Working with you was a very enjoyable and enriching experience! I am very grateful to all the people that shared daily life with me at camp between many conversations and laughs, separated by several sessions of Senegalese tea. Thanks to Susanne for the great food, teaching me how to cook Mafé, and singing and translating the marvelous songs of Toure Kunda together. Thanks to Boudiang for the best boat tour I ever had and for being the soul of the 'Poste de garde de Simenti'. Many thanks go to Boudou, Ndao, Badji, Sadio, Ndiaye, Elisabeth, Kandji, Nabu, Kali, and Pap for the nice chats and time together. A big thanks goes to Sékou for always being so chatty and positive, for the invaluable help with the car and always making me smile! Thanks to Hamadi and Ibraima for always welcoming us in Dialakoto and offering a wonderful break during the travel to Tamba. An enormous thanks goes to the friends in Tambacounda. Ibu Diatta, thank you for always being in contact and welcoming us in your home for the Tabaski and many other occasions. Claire thank you for the long conversations about Senegalese culture, the good and the bad, the latest series to watch, and the many marvelous banana ice creams! Mam Sadio thank you for your kindness, the time spent together, and the feminine company in the field and in Tamba. Massamba, thank you for sewing gorgeous dresses, discussing and laughing about my drawings and design projects, and most of for your patience! Zakaria, a big thanks for being my absolute favorite Tambacounda routine. Your amazing breakfast kept me fed and happy. The time in your "Tangana" chatting and enjoying meals with people is one of my best memories! Coura, what to say, we met many years ago when I bought fruit for the first time and could barely speak a word of French. I am in great debt for all the kindness, smiles and affection during the tough times and for the amazing fruit and juices that kept me heathy! I am also grateful to Oumar and Tabby Ndiaye for the time spent in the field, the trip to Kedougou and Dindéfélo, sharing many projects together and our friendship. A special thanks goes to Taye for being a long-term friend, for the laughs, the fun, the chats and gossiping sessions, and for showing me how strong a woman could be despite difficulties!

I want to thank all the colleagues of the Cognitive Ethology Lab for being such a nice working group including Dietmar, Kurt, Adeelia, Matze, Laura, Steffi, Davide, Caro, Domingo, Lucas, Rowan, Vanessa, Magdalena, Anais, Dominique, Lena, and Ute. A special thanks goes to Ludwig for the IT support, Italian quality caffè, and nice and funny chats during my time in the lab. Mechthild, thank you for the help with the German administration and the sweets and amazing cakes. Franzi Trede, thank you so much for the invaluable help with the genetic analysis and the amazing job. I am grateful for the hours spent discussing the lab details, analyzing the data together and your patience in explaining me things sometimes over and over again. Eva, thank you for being a very good office mate, full of advice and tips, and for the many chatty moments we enjoyed together! Filipa, thank you for the nice chats and discussions trying to figure out complex stats together and improving our analyses! Sarah, what to say... thank you for all the time spent having fun together, for being a friend 
and bringing Italian vibes into my daily life! I deeply enjoyed our coffees and aperitivi accompanied by lengthy discussion about life, love and funny cultural anecdotes.

A big thank you goes to all the people of the old Göttingen PhD+ crowd for having welcomed me so warmly when I was still a master student and for becoming an important part of my life! I especially want to thank Marlies for all the fun, dinners, Zumba sessions, yoga routines, being a wonderful friend and much more! Sally and Fred, thank you for the Göttingen visits and time in Konstanz, the long chats about the world, life, and the future! Flavia, the short time spent together was so much fun and so full of laughs. Getting to know you was awesome and I really hope to be on the same continent again soon! Josi, thank you for the many long chats always ending in the middle of the night. Most of all, thank you for the field discussions, the conversations about Senegal and for keeping me updated with the baboon life. This made me feel as if I was there with the monkeys again, thanks! Cedric and Andreas, thank you for the nice chats and the interesting conversations about science and research. Thanks to Philip and Kirsten for the nice time and outings together. Barbara and Brandon, thank you for welcoming us back from the field and for the wonderful and relaxed time together! It is always a pleasure to see you!

I want to thank all the new Göttingen PhD/DPZ crowd for the many events and social activities shared together! Thank you to Delphine, Alan, and Lauren for these years together and always being there through the tough and good times. I will never forget all the chats and fun we had! I also want to thank Anna, Franzi, Charlotte, Nadine, Anja, Louise, Johanna, Adi, Simon, Sonia, Simone, Sofia and many more for the knitting sessions, hula hoop lessons, the many DPZ lunches, drinks, dinners and many BBQs. It was a lot of fun! Thanks to Ralf and Dana for the Sunday walks plus coffee and cake, the game nights, and Ralf's hilarious jokes! Thank you Antonio and Rebecca for being my Italian connection, the many delicious meals and many talks about babies and family life!

I would like to give a special thank you to all my family and friends who always supported and encouraged me in my crazy life adventures and projects and that always loved me. Thanks to Greta for being the closest friend I ever had and sharing life at a distance with me throughout these many years and to Celia for being who she is and being like a sister to me. Thanks to Matteo, Elena, Carlotta, and many other unforgettable friends for never losing touch and being reliable anchors in my life. I want to thank Meissa for her powerful personality that helped me throughout my Senegalese experience, for having welcomed me in Dakar, and sharing such a gorgeous and funny time together. A special thank you goes to Sandrino, Marta and Hans and to many other family friends without whom I would not be as I am today! I also deeply thank my entire family (nonno Giorgio, nonna Vittoria, zia Luisa, zii Guiliano e Maria, nonna Giovanna) for loving, accepting, and supporting me all the while being confused about my choices of living abroad or in the "jungle" following monkeys. I want to thank my extended family (Traute, Imme, Jacques, Malin, Hannah, and many more) for being in my life. A special thank you goes to my wonderful parents, Laura and Walther, from whom I got the curiosity and the excitement for the things around me. Thank you for all the traveling and 
cultural experiences we lived together that shape me as a person. Thanks for always letting me develop and make choices freely and independently, for always being there for me, for constantly being able to find a bright and funny note even in the most difficult moments, and for all the fun and laughter!

The most special thank goes to Matthis, my husband and partner in life. The list of things I would like to thank you for is far too long. Thank you for everything! I am grateful to have been able to share these unforgettable years together, to be a great team in life and work, to be able to share everything so spontaneously and openly, to the crazy and long discussions about science, the world, our life and how we got to be who we are, our dreams and how we want to get to where we want to be, ourselves and our biggest fears and loves. Thank you for being in my life, for your support through the thick and thin of the $\mathrm{PhD}$ stress, for all the amazing scientific discussions that made this experience so much more valuable, and for all the fun and laughs during the years. The best is yet to come! 


\section{Curriculum Vitae}

This information is available in the printed version of the manuscript. 

...and thank you to the Guinea baboons!

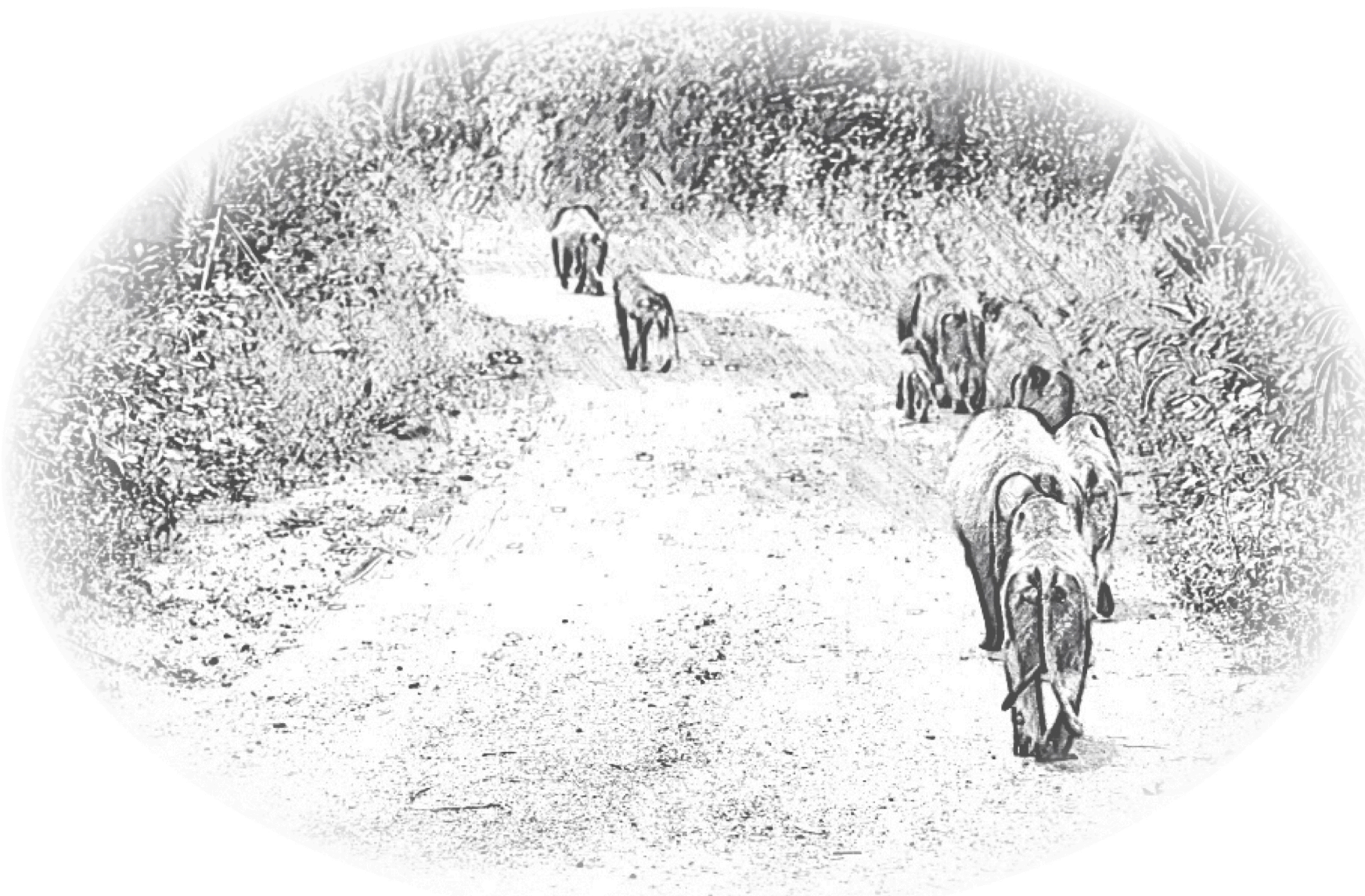

